

\title{
Minimisation and reduction of 2-, 3- and 4-coverings of elliptic curves
}

\author{
John E. Cremona, Tom A. Fisher and Michael Stoll
}

We consider models for genus-one curves of degree $n$ for $n=2,3$ and 4, which arise in explicit $n$-descent on elliptic curves. We prove theorems on the existence of minimal models with the same invariants as the minimal model of the Jacobian elliptic curve and provide simple algorithms for minimising a given model, valid over general number fields. Finally, for genus-one models defined over $\mathbb{Q}$, we develop a theory of reduction and again give explicit algorithms for $n=2,3$ and 4 .

1. Introduction

2. Genus-one models 765

3. Minimisation theorems 770

4. Minimisation algorithms 778

5. Minimisation of insoluble genus-one models 793

6. Reduction 801

7. Examples 810

$\begin{array}{ll}\text { References } & 817\end{array}$

\section{Introduction}

Let $E$ be an elliptic curve defined over a number field $K$. An $n$-descent on $E$ computes the $n$-Selmer group $\operatorname{Sel}^{(n)}(K, E)$ of $E$, which parametrises the everywhere locally soluble $n$-coverings of $E$ up to isomorphism. An $n$-covering of $E$ is a principal homogeneous space $C$ for $E$, together with a map $\pi: C \rightarrow E$ that fits into a commutative diagram

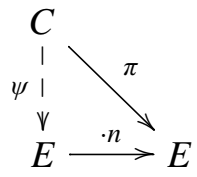

where $\psi: C \rightarrow E$ is an isomorphism defined over the algebraic closure $\bar{K}$, compatible with the structure of $C$ as a principal homogeneous space. In a series of papers

MSC2000: primary 11G05; secondary $11 \mathrm{G} 07,11 \mathrm{G} 05,14 \mathrm{H} 52,14 \mathrm{H} 25$.

Keywords: elliptic curves, genus-one curves, minimisation, reduction, descent. 
[Cremona et al. 2008; 2009; n.d.], it is shown how to produce explicit equations of covering curves from a more abstract representation of the Selmer group. (The latter is computed, at least for $n$ prime, in [Schaefer and Stoll 2004].)

In general, an $n$-covering $C$ can be realised as a smooth curve of degree $n$ inside a Severi-Brauer variety $S$ of dimension $n-1$ (when $n=2$, we obtain a double cover of a conic instead of an embedding). If $C$ has points everywhere locally, as will be the case when $C$ represents an element of the $n$-Selmer group of $E$, then the same statement is true of $S$, and hence $S \cong \mathbb{P}^{n-1}$, so that $C$ has a degree- $n$ model in projective space. Thus, for $n=2$, we get a double cover of $\mathbb{P}^{1}$ ramified in four points, for $n=3$, we get a plane cubic curve, and for $n=4$, we get an intersection of two quadrics in $\mathbb{P}^{3}$. For larger $n$, these models are no longer complete intersections, but can be given by a number of quadratic equations.

In this paper, we will focus on the problem of how to produce nice models of the covering curves, i.e., models given by equations with small integral coefficients, in the cases $n=2,3$ and 4. The advantage of having such a nice model is two-fold. On the one hand, rational points on the covering curve can be expected to be of smaller height on a model with small coefficients, and therefore will be found more easily. On the other hand, if no rational points are found, one would like to use the covering curve as the basis for a further descent, and the necessary computations are greatly facilitated when the given model is nice.

This problem naturally splits into two parts: minimisation and reduction. Minimisation makes the invariants of the model smaller by eliminating spurious bad primes and reducing the exponents of primes of bad reduction, to obtain a socalled minimal model. We will prove the following theorem. (See Section 2 for the definitions of models for $n$-coverings and their invariants.)

Theorem 1.1. Let $n=2,3$ or 4 . Let $K$ be a number field of class number one, and $E$ an elliptic curve defined over $K$. If $\mathscr{b}$ is an $n$-covering of $E$ which is everywhere locally soluble (i.e., $\mathscr{C}$ has points over all completions of $K$ ) then $\mathscr{C}$ has a model with integral coefficients and the same discriminant as a global minimal Weierstrass equation for $E$.

By contrast, reduction attempts to reduce the size of the coefficients by an invertible integral (i.e., unimodular) linear change of coordinates, which leaves the invariants unchanged. Both processes are necessary to obtain a nice model: minimisation without reduction will provide a model with small invariants, but most likely rather large coefficients, whereas reduction without minimisation will not be able to make the coefficients really small, since the invariants will still be large.

After introducing the kinds of models we will be using and their invariants in Section 2, we state our main results on minimisation over local fields in Section 3A, and discuss how they relate to earlier work. The most important of these results 
(the Minimisation Theorem, Theorem 3.4) is proved in Section 3B. The proof is short and transparent, but is not algorithmic. We remedy this in Section 4 where we give practical algorithms for computing minimal models, that may be seen as generalising Tate's algorithm [1975]. In Section 4E we deduce Theorem 1.1 from our local results, and explain how it may be generalised to arbitrary number fields. Moreover, as our local minimisation results make no restriction on the characteristic of the local field, they have more general global applications; in particular, one obtains results over function fields as well as number fields.

The algorithms of Section 4 may be combined with the Minimisation Theorem to prove the Strong Minimisation Theorem, Theorem 3.5(i). This states that if an $n$-covering of $E$ (defined over a local field, and represented by a degree- $n$ model) is soluble over the maximal unramified extension, then it has a model with integral coefficients and the same discriminant as a minimal Weierstrass equation for $E$. In Section 5 we prove the converse, Theorem 3.5(ii), thereby showing that the Strong Minimisation Theorem is best possible.

In Section 6, we discuss reduction for general $n$-coverings, and more specifically for $n=2,3$ and 4. Our results for reduction only cover the case where the ground field is $\mathbb{Q}$. A comparable theory of reduction over a general number field would be very useful in practice, but has not yet been sufficiently developed.

Finally we give examples of both minimisation and reduction (over $K=\mathbb{Q}$ ) in Section 7. Our algorithms have all been implemented over $\mathbb{Q}$ in MAGMA [Bosma et al. 1997] for $n=2,3$ and 4 .

As stated earlier, the main application of our results is in explicit $n$-descent on elliptic curves over number fields. Minimisation and reduction of binary quartics is also used in the invariant theory method for 2-descent [Birch and Swinnerton-Dyer 1963; Cremona 1997]. For $n=3$, Djabri and Smart [1998] consider the possibility of carrying out 3-descent using invariant theory in a similar way; one stumblingblock there was the inability to minimise plane cubic models for 3-coverings.

\section{Genus-one models}

In this section, we specify the models of the covering curves that we will use, together with their invariants $c_{4}, c_{6}$ and $\Delta$. For completeness and later reference we include the case $n=1$. Note that we use the term genus-one model to include singular models, which do not define curves of genus one.

Definition 2.1. A Weierstrass equation, or genus-one model of degree 1, is an equation of the form

$$
y^{2}+a_{1} x y+a_{3} y=x^{3}+a_{2} x^{2}+a_{4} x+a_{6} .
$$


The space of all Weierstrass equations with coefficients $a_{1}, \ldots, a_{6}$ in a ring $R$ will be denoted $X_{1}(R)$. We say that two such models are $R$-equivalent if they are related by substitutions

$$
x \leftarrow u^{2} x+r, \quad y \leftarrow u^{3} y+u^{2} s x+t,
$$

for some $u \in R^{\times}$and $r, s, t \in R$. We write $\mathscr{G}_{1}(R)$ for the group of all transformations $[u ; r, s, t]$ and define $\operatorname{det}([u ; r, s, t])=u^{-1}$. The invariants $c_{4}, c_{6}$ and $\Delta$ are certain primitive polynomials in $a_{1}, \ldots, a_{6}$ with integer coefficients, satisfying

$$
c_{4}^{3}-c_{6}^{2}=1728 \Delta .
$$

Definition 2.2. A genus-one model of degree 2, or generalised binary quartic, is an equation of the form

$$
y^{2}+P(x, z) y=Q(x, z)
$$

where $P$ and $Q$ are homogeneous polynomials of degrees 2 and 4 . We sometimes abbreviate this as $(P, Q)$. The space of all such models with coefficients in a ring $R$ is denoted $X_{2}(R)$. Two such models are $R$-equivalent if they are related by substitutions $x \leftarrow m_{11} x+m_{21} z, z \leftarrow m_{12} x+m_{22} z$ and $y \leftarrow \mu^{-1} y+r_{0} x^{2}+r_{1} x z+r_{2} z^{2}$ for some $\mu \in R^{\times}, r=\left(r_{0}, r_{1}, r_{2}\right) \in R^{3}$ and $M=\left(m_{i j}\right) \in \mathrm{GL}_{2}(R)$. We write $\mathscr{G}_{2}(R)$ for the group of all such transformations $[\mu, r, M]$, and define

$$
\operatorname{det}([\mu, r, M])=\mu \operatorname{det}(M) \text {. }
$$

A generalised binary quartic $y^{2}+P\left(x_{1}, x_{2}\right) y=Q\left(x_{1}, x_{2}\right)$ over a field $K$ defines a subscheme $\mathscr{C}_{(P, Q)} \subset \mathbb{P}(1,1,2)$, the ambient space being a weighted projective space with coordinates $x_{1}, x_{2}, y$. We say that the model $\Phi=(P, Q)$ is $K$-soluble if $\mathscr{C}_{\Phi}(K) \neq \varnothing$.

The binary quartic $F(x, z)=a x^{4}+b x^{3} z+c x^{2} z^{2}+d x z^{3}+e z^{4}$ has invariants $c_{4}(F)=2^{4} I$ and $c_{6}(F)=2^{5} J$, where $I$ and $J$ are given by

$$
I=12 a e-3 b d+c^{2}, \quad J=72 a c e-27 a d^{2}-27 b^{2} e+9 b c d-2 c^{3} .
$$

The discriminant $\Delta=\left(c_{4}^{3}-c_{6}^{2}\right) / 1728$ is 16 times the usual discriminant of a quartic polynomial. The invariants of a generalised binary quartic are obtained by completing the square, i.e., $c_{4}(P, Q)=c_{4}\left(\frac{1}{4} P^{2}+Q\right)$ and so on. We find that $c_{4}, c_{6}$ and $\Delta$ are primitive integer coefficient polynomials in the coefficients of $P$ and $Q$, again satisfying $c_{4}^{3}-c_{6}^{2}=1728 \Delta$.

Earlier work on 2-coverings, including [Birch and Swinnerton-Dyer 1963] and [Stoll and Cremona 2002], used the more restrictive binary quartic models with $P=0$. We use generalised binary quartics here, in order to obtain more uniform local results at places with residue characteristic 2 . 
Definition 2.3. A genus-one model of degree 3 is a ternary cubic. We write $X_{3}(R)$ for the space of all ternary cubics with coefficients in a ring $R$. Two such models are $R$-equivalent if they are related by multiplying by $\mu \in R^{\times}$and then substituting $x_{j} \leftarrow \sum_{i=1}^{3} m_{i j} x_{i}$ for some $M=\left(m_{i j}\right) \in \mathrm{GL}_{3}(R)$. We write $\varphi_{3}(R)=R^{\times} \times \mathrm{GL}_{3}(R)$ for the group of all such transformations $[\mu, M]$, and we define

$$
\operatorname{det}([\mu, M])=\mu \operatorname{det}(M) .
$$

A ternary cubic $F(x, y, z)$ over a field $K$ defines a subscheme $\mathscr{C}_{F} \subset \mathbb{P}^{2}$. The model $F$ is $K$-soluble if $\mathscr{b}_{F}(K) \neq \varnothing$.

The invariants $c_{4}$ and $c_{6}$ may be defined as follows. Let

$$
H(F)=\operatorname{det}\left(\begin{array}{lll}
F_{x x} & F_{x y} & F_{x z} \\
F_{y x} & F_{y y} & F_{y z} \\
F_{z x} & F_{z y} & F_{z z}
\end{array}\right)
$$

be the Hessian of $F$, which is again a ternary cubic. Then we have

$$
H(H(F))=48 c_{4}(F)^{2} F+16 c_{6}(F) H(F) ;
$$

the sign of $c_{4}(F)$ is fixed by requiring that $\Delta=\left(c_{4}^{3}-c_{6}^{2}\right) / 1728$ has integer coefficients. Then $c_{4}, c_{6}$ and $\Delta$ are primitive integer coefficient polynomials in the coefficients of $F$ and satisfy $c_{4}^{3}-c_{6}^{2}=1728 \Delta$.

Definition 2.4. A genus-one model of degree 4, or quadric intersection, is an ordered pair $\left(Q_{1}, Q_{2}\right)$ of quadrics (homogeneous polynomials of degree 2) in 4 variables. The space of all such models with coefficients in a ring $R$ is denoted $X_{4}(R)$. Quadric intersections $\left(Q_{1}, Q_{2}\right)$ and $\left(Q_{1}^{\prime}, Q_{2}^{\prime}\right)$ are $R$-equivalent if they are related by putting $Q_{1}^{\prime}=m_{11} Q_{1}+m_{12} Q_{2}$ and $Q_{2}^{\prime}=m_{21} Q_{1}+m_{22} Q_{2}$ for some $M=\left(m_{i j}\right) \in \mathrm{GL}_{2}(R)$ and then substituting $x_{j} \leftarrow \sum_{i=1}^{4} n_{i j} x_{i}$ for some $N=\left(n_{i j}\right) \in \mathrm{GL}_{4}(R)$. We write $\varphi_{4}(R)=\mathrm{GL}_{2}(R) \times \mathrm{GL}_{4}(R)$ for the group of all such transformations $[M, N]$, and $\operatorname{define} \operatorname{det}([M, N])=\operatorname{det}(M) \operatorname{det}(N)$.

A quadric intersection $\Phi=\left(Q_{1}, Q_{2}\right)$ over a field $K$ defines a subscheme $\mathscr{C}_{\Phi}$ of $\mathbb{P}^{3}$. The model $\Phi$ is $K$-soluble if $\mathscr{C}_{\Phi}(K) \neq \varnothing$.

The invariants $c_{4}$ and $c_{6}$ may be defined as follows. Let $A$ and $B$ be the matrices of second partial derivatives of $Q_{1}$ and $Q_{2}$. Then $F(x, z)=\operatorname{det}(A x+B z)$ is a binary quartic. We define $c_{4}\left(Q_{1}, Q_{2}\right)=2^{-4} c_{4}(F), c_{6}\left(Q_{1}, Q_{2}\right)=2^{-6} c_{6}(F)$ and $\Delta\left(Q_{1}, Q_{2}\right)=2^{-12} \Delta(F)$. These scalings are chosen so that $c_{4}, c_{6}$ and $\Delta$ are primitive integer coefficient polynomials in the coefficients of $Q_{1}$ and $Q_{2}$. They satisfy $c_{4}^{3}-c_{6}^{2}=1728 \Delta$.

Earlier work on 4-coverings, including [Siksek 1995] and [Womack 2003], used pairs of symmetric matrices rather the pairs of quadrics. We use quadrics here, in order to obtain more uniform local results at places with residue characteristic 2 . 
Remark 2.5. There is also a definition of genus-one model of degree 5; see [Fisher 2008a]. The minimisation and reduction of these models (and possible extensions to larger degrees) will be the subject of future investigations.

Remark 2.6. There is a natural way in which we can rewrite a Weierstrass equation (a genus-one model of degree 1) as a genus-one model of degree 2, 3 or 4 (see Lemma 3.11). We have normalised the invariants $c_{4}, c_{6}$ and $\Delta$ so that they agree with the usual formulae (see [Silverman 1986, Chapter III], for example) when specialised to one of these Weierstrass models.

Definition 2.7. Let $K$ be a field and $\bar{K}$ its algebraic closure. Let $K\left[X_{n}\right]$ be the polynomial ring in the coefficients of a genus-one model of degree $n$. A polynomial $F \in K\left[X_{n}\right]$ is an invariant of weight $k$ if $F \circ g=\operatorname{det}(g)^{k} F$ for all $g \in \mathscr{G}_{n}(\bar{K})$.

For $n=1,2,3,4$ we defined polynomials $c_{4}, c_{6}, \Delta \in \mathbb{Z}\left[X_{n}\right]$ with

$$
c_{4}^{3}-c_{6}^{2}=1728 \Delta \text {. }
$$

Theorem 2.8. Let $n=1,2,3$ or 4 .

(i) The polynomials $c_{4}, c_{6}, \Delta \in K\left[X_{n}\right]$ are invariants of weights 4,6 and 12 .

(ii) A genus-one model $\Phi \in X_{n}(K)$ defines a smooth curve $\mathscr{C}_{\Phi}$ of genus one (over $\bar{K})$ if and only if $\Delta(\Phi) \neq 0$.

(iii) If $\operatorname{char}(K) \neq 2,3$ then $c_{4}$ and $c_{6}$ generate the ring of invariants. Moreover if $\Phi \in X_{n}(K)$ with $\Delta(\Phi) \neq 0$ then the Jacobian of the curve $\mathscr{C}_{\Phi}$ has Weierstrass equation $y^{2}=x^{3}-27 c_{4}(\Phi) x-54 c_{6}(\Phi)$.

Proof. The invariants $c_{4}, c_{6}$ and $\Delta$ were known to the nineteenth century invariant theorists. The observation that they give a formula for the Jacobian is due to Weil [1954; 1983]. See [An et al. 2001] for a brief survey, or [Fisher 2008a] for a proof of the theorem exactly as it is stated here.

As was first pointed out to us by Rodriguez-Villegas, it is possible to work back through Tate's formulaire [Silverman 1986, Chapter III] to write the invariants $c_{4}$ and $c_{6}$ in terms of polynomials $a_{1}, \ldots, a_{6}$.

Lemma 2.9. There exist $a_{1}, a_{2}, a_{3}, a_{4}, a_{6} \in \mathbb{Z}\left[X_{n}\right]$ and $b_{2}, b_{4}, b_{6} \in \mathbb{Z}\left[X_{n}\right]$ with

$$
\begin{aligned}
& b_{2}=a_{1}^{2}+4 a_{2}, \quad b_{4}=a_{1} a_{3}+2 a_{4}, \quad b_{6}=a_{3}^{2}+4 a_{6}, \\
& c_{4}=b_{2}^{2}-24 b_{4}, \quad c_{6}=-b_{2}^{3}+36 b_{2} b_{4}-216 b_{6} .
\end{aligned}
$$

Proof. The lemma is proved by splitting into the cases $n=2,3,4$ and giving explicit formulae for the $a$-invariants. (The case $n=1$ is a tautology.)

Case $n=2$. The $a$-invariants of the generalised binary quartic,

$$
y^{2}+\left(l x^{2}+m x z+n z^{2}\right) y=a x^{4}+b x^{3} z+c x^{2} z^{2}+d x z^{3}+e z^{4},
$$


are

$$
\begin{aligned}
& a_{1}=m, \quad a_{2}=c-\ln , \quad a_{3}=l d+n b, \\
& a_{4}=-4 a e+b d-\left(l^{2} e+\ln c+n^{2} a\right), \\
& a_{6}=-4 a c e+a d^{2}+b^{2} e-\left(l^{2} c e+m^{2} a e+n^{2} a c+\ln b d\right)+l m b e+\text { mnad. }
\end{aligned}
$$

Case $n=3$. The $a$-invariants of the ternary cubic,

$$
a x^{3}+b y^{3}+c z^{3}+a_{2} x^{2} y+a_{3} x^{2} z+b_{1} x y^{2}+b_{3} y^{2} z+c_{1} x z^{2}+c_{2} y z^{2}+m x y z
$$

$\operatorname{are}^{1}$

$$
\begin{aligned}
& a_{1}=m, \\
& a_{2}=-\left(a_{2} c_{2}+a_{3} b_{3}+b_{1} c_{1}\right), \\
& a_{3}=9 a b c-\left(a b_{3} c_{2}+b a_{3} c_{1}+c a_{2} b_{1}\right)-\left(a_{2} b_{3} c_{1}+a_{3} b_{1} c_{2}\right), \\
& a_{4}=-3\left(a b c_{1} c_{2}+a c b_{1} b_{3}+b c a_{2} a_{3}\right)+a\left(b_{1} c_{2}^{2}+b_{3}^{2} c_{1}\right)+b\left(a_{2} c_{1}^{2}+a_{3}^{2} c_{2}\right) \\
& \quad+c\left(a_{2}^{2} b_{3}+a_{3} b_{1}^{2}\right)+a_{2} c_{2} a_{3} b_{3}+b_{1} c_{1} a_{2} c_{2}+a_{3} b_{3} b_{1} c_{1},
\end{aligned}
$$

$a_{6}=-27 a^{2} b^{2} c^{2}+9 a b c\left(a b_{3} c_{2}+c a_{2} b_{1}+b a_{3} c_{1}\right)+\cdots+a b c m^{3}$.

These formulae in the case $n=3$ were first given in [Artin et al. 2005].

Case $n=4$. Let $Q=\sum_{i \leq j} c_{i j} x_{i} x_{j}$ be a quadric in 4 variables. Then

$$
\operatorname{det}\left(\frac{\partial^{2} Q}{\partial x_{i} \partial x_{j}}\right)=\operatorname{pf}(Q)^{2}+4 \operatorname{rd}(Q)
$$

where $\operatorname{pf}(Q)=c_{12} c_{34}+c_{13} c_{24}+c_{14} c_{23}$ and $\operatorname{rd}(Q) \in \mathbb{Z}\left[c_{11}, c_{12}, \ldots, c_{44}\right]$. We define the $a$-invariants of the quadric intersection $\left(Q_{1}, Q_{2}\right)$ to be the $a$-invariants of the generalised binary quartic

$$
y^{2}+\operatorname{pf}\left(x Q_{1}+z Q_{2}\right) y=\operatorname{rd}\left(x Q_{1}+z Q_{2}\right) .
$$

The polynomials $a_{i}$ of Lemma 2.9 are far from unique. They can be modified by any transformation of the form $[ \pm 1 ; r, s, t]$ with $r, s, t \in \mathbb{Z}\left[X_{n}\right]$. We next extend Theorem 2.8(iii) to fields of arbitrary characteristic. (The reader only interested in applications over number fields and their completions may safely skip this result.)

Theorem 2.10. Let $K$ be any field, and $n=1,2,3$ or 4. For all $\Phi \in X_{n}(K)$ with $\Delta(\Phi) \neq 0$, the Jacobian of the curve $\mathscr{C}_{\Phi}$ has Weierstrass equation

$$
y^{2}+a_{1} x y+a_{3} y=x^{3}+a_{2} x^{2}+a_{4} x+a_{6},
$$

where $a_{i}=a_{i}(\Phi)$.

\footnotetext{
${ }^{1}$ We follow classical notation for the coefficient labels, but warn the reader that the symbols $a_{2}$, $a_{3}$ have different meanings on the left and right sides of (2-4).
} 
Proof. For $n=3$ this is a special case of a theorem of Artin, Rodriguez-Villegas and Tate [2005]. The cases $n=2,4$ may be proved using similar techniques. We sketch a simplified form of the proof, covering the cases $n=2,3$ and 4 . (The case $n=1$ is of course a tautology.)

Let $C / S$ be the universal family over ${ }^{2} S=\operatorname{Spec}\left(\mathbb{Z}\left[X_{n}\right]\left[\Delta^{-1}\right]\right)$. By Theorem 2.8 (ii) the fibres are smooth projective curves of genus one. Let $J / S$ be the Jacobian of $C / S$, in the sense that $J$ is the $S$-scheme representing the relative Picard functor $\mathrm{Pic}_{C / S}^{0}$; see [Bosch et al. 1990, §9.3, Theorem 1]. Each fibre of $J / S$ is the Jacobian of the corresponding fibre of $C / S$ and hence an elliptic curve. By a generalisation of the usual procedure for putting an elliptic curve in Weierstrass form (see [Deligne 1975] or [Artin et al. 2005, Theorem 2] for a further generalisation) $J$ is defined as a subscheme of $\mathbb{P}_{S}^{2}$ by the homogenisation of

$$
y^{2}+a_{1}^{\prime} x y+a_{3}^{\prime} y=x^{3}+a_{2}^{\prime} x^{2}+a_{4}^{\prime} x+a_{6}^{\prime}
$$

for some $a_{1}^{\prime}, \ldots, a_{6}^{\prime} \in \mathbb{Z}\left[X_{n}\right]\left[\Delta^{-1}\right]$. Thus for every field $K$, and every $\Phi \in X_{n}(K)$ with $\Delta(\Phi) \neq 0$, the Weierstrass equation (2-6) gives a model for the Jacobian of $\mathscr{C}_{\Phi}$.

It only remains to show that (2-5) and (2-6) are related by a transformation in $\mathscr{G}_{1}(R)$ where $R=\mathbb{Z}\left[X_{n}\right]\left[\Delta^{-1}\right]$. By Theorem 2.8(iii) they are related by some $[u ; r, s, t] \in \mathscr{G}_{1}(K)$ where $K=\mathbb{Q}\left(X_{n}\right)$. Since for any genus-one model with $\Delta \neq 0$, (2-5) and (2-6) both specialise to a nonsingular Weierstrass equation, it follows that $u \in R^{\times}$. Then, since $R$ is integrally closed, a standard argument (see [Silverman 1986, Chapter VII, Proposition 1.3]) shows that $r, s, t \in R$.

We note that $a_{1}, \ldots, a_{6}$ are not invariants in the sense of Definition 2.7. The ring of invariants when $\operatorname{char}(K)=2$ or 3 is described in [Fisher 2008a, §10]. As is noted there, these do not give a formula for the Jacobian.

\section{Minimisation theorems}

3A. Statement of results. Let $K$ be a field with normalised discrete valuation $v: K^{\times} \rightarrow \mathbb{Z}$. We write $\mathrm{O}_{K}$ for the valuation ring (or ring of integers) of $K$ and fix a uniformiser $\pi \in K$. We assume throughout that the residue field $k=\mathbb{O}_{K} / \pi \mathbb{O}_{K}$ is perfect. A field extension $L / K$ is unramified if there is a (normalised) discrete valuation $w: L^{\times} \rightarrow \mathbb{Z}$ extending $v$. The strict Henselisation $K^{\text {sh }}$ of $K$ is an unramified extension of $K$, that satisfies the conclusions of Hensel's lemma and has residue field $\bar{k}$, the algebraic closure of $k$. (See [Milne 2008, Definition 4.8] or [Raynaud 1970, Chapter VIII] for the precise definition.) If $K$ is complete (with

${ }^{2}$ In [Artin et al. 2005] the authors work over $S=\operatorname{Spec}\left(\mathbb{Z}\left[X_{3}\right]\right) \backslash\{0\}$. This gives a more general result, but also makes the proof more difficult. 
respect to $v$ ) then $K^{\text {sh }}$ is the maximal unramified extension $K^{\mathrm{nr}}$ of $K$ as defined in [Serre 1979, Chapter III, §5].

We work with genus-one models of degree $n=1,2,3$ or 4 . The invariants $c_{4}$, $c_{6}$ and $\Delta$ of a genus-one model were defined in Section 2.

Definition 3.1. (i) A genus-one model $\Phi \in X_{n}(K)$ is nonsingular if $\Delta(\Phi) \neq 0$.

(ii) A genus-one model $\Phi \in X_{n}(K)$ is integral if it has coefficients in $\mathscr{O}_{K}$.

(iii) A nonsingular model $\Phi \in X_{n}\left(\mathbb{O}_{K}\right)$ is minimal if $v(\Delta(\Phi))$ is minimal among all integral models $K$-equivalent to $\Phi$, otherwise $\Phi$ is nonminimal.

Algorithms for computing minimal models in the case $n=1$ have been given by Tate [1975] (see also [Silverman 1994, Chapter IV, §9]) and Laska [1982]. The latter can be refined using Kraus' conditions [Kraus 1989] as described in [Connell 1996, Chapter V] or [Cremona 1997, §3.2]. (Laska's algorithm and its refinements are simpler than Tate's algorithm, but are only applicable when $\operatorname{char}(K) \neq 2$, 3.) In Section 4 we give algorithms for computing minimal models in the cases $n=2,3,4$.

In the following lemma we define the level of a genus-one model.

Lemma 3.2. Let $\Phi \in X_{n}(K)$ be a nonsingular model of degree $n$. Let $\Delta_{E}$ be the minimal discriminant of $E=\operatorname{Jac}\left(\mathscr{C}_{\Phi}\right)$.

(i) $v(\Delta(\Phi))=v\left(\Delta_{E}\right)+12$ l for some integer $l$, which we call the level of $\Phi$.

(ii) If $\operatorname{char}(k) \neq 2,3$ then $l=\min \left\{\left\lfloor v\left(c_{4}(\Phi)\right) / 4\right\rfloor,\left\lfloor v\left(c_{6}(\Phi)\right) / 6\right\rfloor\right\}$.

(iii) The level of an integral model is always nonnegative.

Proof. If $\operatorname{char}(k) \neq 2,3$ then this is clear by Theorem 2.8 and the standard formulae for transforming Weierstrass equations. In general (that is, to prove (iii) when $\operatorname{char}(k)=2$ or 3 , or even to define the level when $\operatorname{char}(K)=2$ or 3 ) we use Lemma 2.9 and Theorem 2.10 instead.

The level of $\Phi \in X_{n}(K)$ may be computed as $v(u)$ where $[u ; r, s, t] \in \mathscr{G}_{1}(K)$ is a transformation that minimises the Weierstrass equation (2-5).

Definition 3.3. The minimal level of $\Phi \in X_{n}(K)$ is the minimum of the levels of all integral models $K$-equivalent to $\Phi$. Thus an integral model $\Phi$ is minimal (see Definition 3.1) if and only if it has level equal to this minimal level.

If $n=1$ then the minimal level is 0 , for trivial reasons. So from now on we take $n=2,3$ or 4 . The most important result on minimisation states that every $K$-soluble model has minimal level 0 , or in other words, that every $K$-soluble model is $K$-equivalent to an integral model whose discriminant has the same valuation as that of the discriminant of the minimal model of the Jacobian elliptic curve. 
Theorem 3.4 (Minimisation Theorem). Let $\Phi \in X_{n}(K)$ be a nonsingular genusone model. If $\mathscr{b}_{\Phi}(K) \neq \varnothing$ then $\Phi$ has minimal level 0 .

The following strengthening of the Minimisation Theorem shows that a nonsingular model is $K$-equivalent to an integral model of level 0 if and only if it is $K^{\text {sh-soluble. }}$

Theorem 3.5. Let $\Phi \in X_{n}(K)$ be nonsingular.

(i) (Strong Minimisation Theorem). If $\mathscr{C}_{\Phi}\left(K^{\mathrm{sh}}\right) \neq \varnothing$ then $\Phi$ has minimal level 0.

(ii) (Converse Theorem). If $\mathscr{C}_{\Phi}\left(K^{\mathrm{sh}}\right)=\varnothing$ then the minimal level is at least 1 , and is equal to 1 if $\operatorname{char}(k) \nmid n$.

Algorithms for minimising $K$-soluble binary quartics over $K=\mathbb{Q}_{p}$ are sketched by Birch and Swinnerton-Dyer [1963, Lemmas 3-5], with details in the case where $p$ is neither 2 nor 3. Their algorithms give a proof of the Minimisation Theorem for $n=2$, except when $p=2$ (in which case further work is required to handle the cross terms). As pointed out in [Stoll and Cremona 2002] this generalises immediately to any local field $K$ with $\operatorname{char}(k) \neq 2,3$. These calculations were extended to the case $n=3$, in conjunction with the authors' work on 3-descent [Cremona et al. 2008; 2009; n.d.]. The case $n=4$ was treated by Womack [2003, Section 2.5], using a method that goes via the results for $n=2$.

In each case, the approach taken is to start with a $K^{\text {sh }}$-soluble model $\Phi \in X_{n}\left(\mathscr{O}_{K}\right)$ with $v\left(c_{4}(\Phi)\right) \geq 4$ and $v\left(c_{6}(\Phi)\right) \geq 6$, and then by a series of substitutions to show that $\Phi$ is $K$-equivalent to an integral model of smaller level. This leads to both a proof of the Strong Minimisation Theorem and a practical algorithm for minimising. However, this traditional approach suffers from the following drawbacks.

- It is necessary to split into a large number of (elementary yet tedious) cases, and the number of cases grows rapidly with $n$.

- The modifications required if $\operatorname{char}(k)=2$ or 3 are somewhat involved. (The hypothesis that $\Phi$ has positive level has to be made explicit using either Kraus' conditions [1989] or the " $a$-invariants" defined in Lemma 2.9.)

We take a different approach, in which the tasks of proving the Minimisation Theorem and finding a practical algorithm for minimising are treated separately. A proof of the Minimisation Theorem for $n=2,3$ (in all residue characteristics) is given in [Fisher 2007]. In Section 3B we simplify the proof and extend it to the case $n=4$. Unfortunately this approach does not lead to any readily implementable algorithm, nor does it prove the Strong Minimisation Theorem.

In Sections 4A (case $n=2$ ) and 4B (case $n=3$ ) we specify a rather simpleminded procedure and show that, given any nonminimal integral model, iterating this procedure will eventually decrease the level. This gives an algorithm for computing minimal models. In Section $4 \mathrm{C}$ we give an algorithm in the case $n=4$ based 
on the treatment in [Womack 2003]. The algorithms for $n=2$ and $n=4$ must be modified when $\operatorname{char}(k)=2$ as described in Section 4D. These modifications are required since, as noted in Section 2, our models for $n$-coverings differ slightly from those used previously in the literature. We have also defined the level, not in an absolute way, but by comparison with a minimal model for the Jacobian elliptic curve. The combined effect of these changes is that our results are much cleaner to state, in particular for residue characteristic 2, and can be proved uniformly, without assumptions on the ramification index.

As is the case for Tate's algorithm, it is clear from the form of our algorithms (for $n=2,3,4$ ) that their success or otherwise is unchanged by an unramified field extension. We deduce the following.

Theorem 3.6. The minimal level of a nonsingular genus-one model of degree 2,3 or 4 is unchanged by an unramified field extension.

The Strong Minimisation Theorem is then an immediate consequence of Theorem 3.6 and the Minimisation Theorem.

In Section 5 we show how to write down examples of minimal genus-one models of positive level. We call the models arising in our construction critical models; see Definition 5.1. We show (for $n=2,3$ ) that any $K^{\text {sh }}$-insoluble model is $K$ equivalent to a critical model. There is a corresponding result for models of degree $n=4$. The proof of the Converse Theorem, 3.5(ii), is then reduced to a statement about the possible levels of a critical model (see Lemma 5.4).

Theorem 3.5 in the case $n=2$ may already be found in [Liu 1996, remarque 21]. We claim that our proof is much simpler, and in any case serves as a template for our generalisations to $n=3,4$. Liu also gives an algorithm for minimising [Liu 1996, p. 4594, remarque 11] (still for $n=2$ ), which although not made explicit appears to be the same as ours.

We remark that minimisations are not unique, in the sense that there can be more than one $\mathrm{O}_{K}$-equivalence class of minimal models $K$-equivalent to a given genusone model. Following on from our work and that of Liu, Sadek [2009] explains how to compute the number of such classes.

For a more general, but necessarily less explicit, discussion of the problem of minimising homogeneous polynomials (of degree $d$ in $n$ variables) see [Kollár 1997].

3B. Proof of the Minimisation Theorem. In this subsection only we relax our assumptions on $\mathrm{O}_{K}$ and $K$. It will only be necessary to assume that $\mathrm{O}_{K}$ is a principal ideal domain and $K$ is its field of fractions. The definitions of a nonsingular model and an integral model (see Definition 3.1) carry over as before. We consider models of degree $n=2,3$ or 4 . 
Let $E$ be an elliptic curve over $K$, with identity $\mathscr{O}_{E} \in E(K)$, and let $D$ be a $K$-rational divisor on $E$ of degree $n$. We write $[D]$ for the linear equivalence class of $D$. We pick a basis $f_{1}, \ldots, f_{n}$ for the Riemann-Roch space $\mathscr{L}(D)$, and let $E \rightarrow \mathbb{P}^{n-1}$ be the morphism given by $P \mapsto\left(f_{1}(P): \ldots: f_{n}(P)\right)$. Then according as $n=2,3$ or 4 , we find that $E$ may be written as either a double cover of $\mathbb{P}^{1}$, a plane cubic, or an intersection of two quadrics in $\mathbb{P}^{3}$. It is therefore defined by a suitable genus-one model $\Phi \in X_{n}(K)$. Moreover this model is uniquely determined up to $K$-equivalence by the pair $(E,[D])$ : replacing $D$ by an equivalent divisor or changing basis for the space $\mathscr{L}(D)$ only has the effect of a linear change of coordinates on $\mathbb{P}^{n-1}$, so only changes the genus-one model by a $K$-equivalence. In this situation we say that the genus-one model $\Phi$ represents the pair $(E,[D])$.

Similarly, we obtain a genus-one model $\Phi \in X_{n}(K)$, well-defined up to $K$ equivalence, representing every pair $(\mathscr{C},[D])$ where $\mathscr{C}$ is a genus-one curve and $D$ a divisor of degree $n$ on $\mathscr{C}$; we have $\mathscr{C} \cong \mathscr{C}_{\Phi}$ (over $K$ ), and in particular, $\Phi$ is $K$-soluble if and only if $\mathscr{C}(K) \neq \varnothing$. Under this isomorphism, the divisor class [D] on $\mathscr{C}$ maps to a distinguished divisor class $\left[D_{\Phi}\right]$ of degree $n$ on $\mathscr{C}_{\Phi}$, namely the class of the fibres of the map $\mathscr{C}_{\Phi} \rightarrow \mathbb{P}^{1}$ if $n=2$, or the hyperplane section if $n=3,4$. It is a tautology that $\Phi$ represents $\left(\mathscr{C}_{\Phi},\left[D_{\Phi}\right]\right)$.

Lemma 3.7. Every $K$-soluble nonsingular genus-one model arises from a pair $(E,[D])$ in the manner described above.

Proof. If $\Phi \in X_{n}(K)$ is a $K$-soluble nonsingular model then $\mathscr{C}_{\Phi}$ is a smooth curve of genus one with a rational point, hence is an elliptic curve. Now it is obvious that the genus-one model determined by the pair $\left(\mathscr{C}_{\Phi},\left[D_{\Phi}\right]\right)$ is just $\Phi$.

The aim of this section is to prove the following theorem. The Minimisation Theorem, 3.4, is then an immediate consequence by Lemma 3.7.

Theorem 3.8. Let $E / K$ be an elliptic curve with integral Weierstrass equation

$$
y^{2}+a_{1} x y+a_{3} y=x^{3}+a_{2} x^{2}+a_{4} x+a_{6}
$$

and let $D \in \operatorname{Div}_{K}(E)$ be a divisor on $E$ of degree $n=2,3$ or 4 . Then $(E,[D])$ can be represented by an integral genus-one model with the same discriminant as (3-1).

This theorem states that, in the $K$-equivalence class of genus-one models representing $(E,[D])$, there is one which is integral and has the same discriminant as any given integral Weierstrass model for $E$. Our strategy for proving this starts with two observations.

Firstly, the claim really does only depend on the divisor class $[D]$ and not the given specific divisor $D$ in that class, since the $K$-equivalence class of genus-one models representing $(E,[D])$ only depends on the divisor class. 
Secondly, if $\tau_{Q}: E \rightarrow E$ is translation by some point $Q \in E(K)$, then the pairs $(E,[D])$ and $\left(E,\left[\tau_{Q}^{*} D\right]\right)$ determine $K$-equivalent genus-one models. This follows from the fact that the map $E \rightarrow \mathbb{P}^{n-1}$ determined by $\left[\tau_{Q}^{*} D\right]$ is the composite of $\tau_{Q}$ and the map determined by $[D]$.

Using the classical facts that every $K$-rational divisor $D$ of degree $n$ is linearly equivalent to a unique divisor of the form $(n-1) \cdot 0_{E}+P$ for some $P \in E(K)$, and that divisors on an elliptic curve are linearly equivalent if and only if they have the same degree and the same sum, it suffices to prove Theorem 3.8 for such divisors as $P$ runs over a set of coset representatives for $E(K) / n E(K)$.

In Lemmas 3.11 and 3.12, we show using explicit formulae that Theorem 3.8 holds in the cases $D=n \cdot 0_{E}$ and $D=(n-1) \cdot 0_{E}+P$ where $P \in E(K)$ is an integral point, that is, a point with coordinates in $\mathbb{O}_{K}$. This is already enough to prove Theorem 3.8 in the case $\mathrm{O}_{K}$ is a complete discrete valuation ring with residue characteristic prime to $n$. Indeed, by the theory of formal groups, every nonzero element of $E(K) / n E(K)$ may then be represented by an integral point.

In general we rely on the following two lemmas, proved later in this subsection.

Lemma 3.9 (unprojection lemma). Let $D \in \operatorname{Div}_{K}(E)$ have degree 2 or 3 , and let $P \in E(K)$. If Theorem 3.8 holds for $D$ then it holds for $D+P$.

Lemma 3.10 (projection lemma). Let $D \in \operatorname{Div}_{K}(E)$ have degree 3 or 4 , and let $P \in E(K)$. If Theorem 3.8 holds for $D$ then it holds for $D-P$.

Theorem 3.8 may be deduced from these lemmas in more than one way. For example, if $n=3$ or 4 then $D \sim(n-1) .0_{E}+P$ for some $P \in E(K)$. Then we quote the result for $D^{\prime}=(n-1) \cdot 0_{E}$ and use the unprojection lemma. Likewise if $n=2$ or 3 then $D \sim(n+1) .0_{E}-P$ for some $P \in E(K)$. Then we quote the result for $D^{\prime}=(n+1) \cdot \mathscr{O}_{E}$ and apply the projection lemma to $D^{\prime}$.

Theorem 3.8 in the case $D=n .0_{E}$ follows from the formulae we used to normalise the invariants $c_{4}, c_{6}$ and $\Delta$ : see Remark 2.6.

Lemma 3.11. Let $E$ be an elliptic curve with Weierstrass equation

$$
Y^{2}+a_{1} X Y+a_{3} Y=X^{3}+a_{2} X^{2}+a_{4} X+a_{6} .
$$

Then the pair $\left(E,\left[n . \mathscr{O}_{E}\right]\right)$ determines genus-one models as follows:

$$
\begin{array}{ll}
n=2: & y^{2}+\left(a_{1} x_{1} x_{2}+a_{3} x_{2}^{2}\right) y=x_{1}^{3} x_{2}+a_{2} x_{1}^{2} x_{2}^{2}+a_{4} x_{1} x_{2}^{3}+a_{6} x_{2}^{4} ; \\
n=3: & y^{2} z+a_{1} x y z+a_{3} y z^{2}-x^{3}-a_{2} x^{2} z-a_{4} x z^{2}-a_{6} z^{3}=0 ; \\
n=4: & x^{2}-z t=0, \quad y^{2}+a_{1} x y+a_{3} y z-x t-a_{2} x^{2}-a_{4} x z-a_{6} z^{2}=0 .
\end{array}
$$

Moreover, each of these models has the same invariants $c_{4}, c_{6}$ and $\Delta$ as (3-2).

Proof. In the case $n=2$ we embed $E$ in $\mathbb{P}(1,1,2)$ via $\left(x_{1}: x_{2}: y\right)=(X: 1: Y)$. In the cases $n=3,4$ we embed $E$ in $\mathbb{P}^{n-1}$ via $(z: x: y)=(1: X: Y)$ and 
$(z: x: y: t)=\left(1: X: Y: X^{2}\right)$ respectively. The statement about the invariants follows by direct calculation.

Next we prove Theorem 3.8 in the case $D=(n-1) .0_{E}+P$ where $P \in E(K)$ is an integral point. By a substitution $X \leftarrow X+X(P), Y \leftarrow Y+Y(P)$ we may assume that $P$ is the point $(0,0)$.

Lemma 3.12. Let $E$ be an elliptic curve with Weierstrass equation

$$
Y^{2}+a_{1} X Y+a_{3} Y=X^{3}+a_{2} X^{2}+a_{4} X
$$

and let $P=(0,0)$. Then the pair $\left(E,\left[(n-1) \cdot 0_{E}+P\right]\right)$ determines genus-one models as follows:

$$
\begin{array}{ll}
n=2: & y^{2}+\left(-x_{1}^{2}+a_{1} x_{1} x_{2}+a_{2} x_{2}^{2}\right) y=-a_{3} x_{1} x_{2}^{3}-a_{4} x_{2}^{4} \\
n=3: & y^{2} z-x^{2} y+a_{1} x y z+a_{2} y z^{2}+a_{3} x z^{2}+a_{4} z^{3}=0 \\
n=4: & z t-x y+a_{1} y z+a_{3} z^{2}=0, \quad y^{2}-x t+a_{2} y z+a_{4} z^{2}=0 .
\end{array}
$$

Moreover, each of these models has the same invariants $c_{4}, c_{6}$ and $\Delta$ as (3-3).

Proof. The rational function

$$
F=\frac{Y+a_{1} X+a_{3}}{X}=\frac{X^{2}+a_{2} X+a_{4}}{Y}
$$

belongs to the Riemann-Roch space $\mathscr{L}\left(\mathcal{O}_{E}+P\right)$. In the case $n=2$ we embed $E$ in $\mathbb{P}(1,1,2)$ via $\left(x_{1}: x_{2}: y\right)=(F: 1: X)$. In the cases $n=3,4$ we embed $E$ in $\mathbb{P}^{n-1}$ via $(z: x: y)=(1: F: X)$ and $(z: x: y: t)=(1: F: X: Y)$ respectively. The statement about the invariants follows by direct calculation.

It remains to prove Lemmas 3.9 and 3.10. One observation that we use in the proofs is the following.

Lemma 3.13. The group $\mathrm{SL}_{n}\left(\mathrm{O}_{K}\right)$ acts transitively on $\mathbb{P}^{n-1}(K)$.

Proof. Since $\mathrm{O}_{K}$ is a principal ideal domain this is standard. See for example [Jacobson 1985, Exercise 6, p. 186].

We now explain how we pass between results for generalised binary quartics (case $n=2$ ) and ternary cubics (case $n=3$ ).

Lemma 3.14. Let $D \in \operatorname{Div}_{K}(E)$ be a divisor of degree 2 and let $P \in E(K)$. Let $f_{1}, f_{2}$ and $f_{3}$ be binary forms over $K$ with $\operatorname{deg} f_{i}=i$. The following statements are equivalent.

(i) The pair $(E,[D])$ is represented by the generalised binary quartic

$$
y^{2}+f_{2}\left(x_{1}, x_{2}\right) y=f_{1}\left(x_{1}, x_{2}\right) f_{3}\left(x_{1}, x_{2}\right)
$$

and $P$ is the point defined by $f_{1}=y=0$. 
(ii) The pair $(E,[D+P])$ is represented by the ternary cubic

$$
f_{1}(X, Z) Y^{2}-f_{2}(X, Z) Y-f_{3}(X, Z)=0
$$

and $P$ is the point $(X: Y: Z)=(0: 1: 0)$.

Proof. We first show that the curves $C_{2}$ and $C_{3}$ defined by (3-4) and (3-5) are isomorphic. An isomorphism $\phi: C_{2} \rightarrow C_{3}$ is given by

$$
\begin{aligned}
\phi:\left(x_{1}: x_{2}: y\right) \mapsto(X: Y: Z) & =\left(x_{1} f_{1}\left(x_{1}, x_{2}\right): y+f_{2}\left(x_{1}, x_{2}\right): x_{2} f_{1}\left(x_{1}, x_{2}\right)\right) \\
& =\left(x_{1} y: f_{3}\left(x_{1}, x_{2}\right): x_{2} y\right),
\end{aligned}
$$

with inverse

$$
\phi^{-1}:(X: Y: Z) \mapsto\left(x_{1}: x_{2}: y\right)=\left(X: Z: f_{1}(X, Z) Y-f_{2}(X, Z)\right) .
$$

The isomorphism identifies the points $\left\{f_{1}=y=0\right\} \in C_{2}(K)$ and $(0: 1: 0) \in C_{3}(K)$. To prove the equivalence of (i) and (ii) we note that if $D=P_{1}+P_{2}$ is a fibre of the map $C_{2} \rightarrow \mathbb{P}^{1} ;\left(x_{1}: x_{2}: y\right) \mapsto\left(x_{1}: x_{2}\right)$ then the points $\phi\left(P_{1}\right), \phi\left(P_{2}\right)$ and $(0: 1: 0)$ are collinear on $C_{3} \subset \mathbb{P}^{2}$.

There is an entirely analogous result for passing between ternary cubics (case $n=3$ ) and quadric intersections (case $n=4$ ).

Lemma 3.15. Let $D \in \operatorname{Div}_{K}(E)$ be a divisor of degree 3 and let $P \in E(K)$. Let $l_{1}$, $l_{2}, q_{1}$ and $q_{2}$ be ternary forms over $K$ with $\operatorname{deg} l_{i}=1$ and $\operatorname{deg} q_{i}=2$. The following statements are equivalent.

(i) The pair $(E,[D])$ is represented by the ternary cubic

$$
l_{1}\left(x_{1}, x_{2}, x_{3}\right) q_{2}\left(x_{1}, x_{2}, x_{3}\right)-l_{2}\left(x_{1}, x_{2}, x_{3}\right) q_{1}\left(x_{1}, x_{2}, x_{3}\right)=0,
$$

and $P$ is the point defined by $l_{1}=l_{2}=0$.

(ii) The pair $(E,[D+P])$ is represented by the quadric intersection

$$
l_{1}\left(x_{1}, x_{2}, x_{3}\right) x_{4}+q_{1}\left(x_{1}, x_{2}, x_{3}\right)=0, \quad l_{2}\left(x_{1}, x_{2}, x_{3}\right) x_{4}+q_{2}\left(x_{1}, x_{2}, x_{3}\right)=0,
$$

and $P$ is the point $\left(x_{1}: x_{2}: x_{3}: x_{4}\right)=(0: 0: 0: 1)$.

Proof. We first show that the curves $C_{3}$ and $C_{4}$ defined by (3-6) and (3-7) are isomorphic. An isomorphism $\phi: C_{3} \rightarrow C_{4}$ is given by

$$
\phi:\left(x_{1}: x_{2}: x_{3}\right) \mapsto\left(x_{1} l_{1}: x_{2} l_{1}: x_{3} l_{1}:-q_{1}\right)=\left(x_{1} l_{2}: x_{2} l_{2}: x_{3} l_{2}:-q_{2}\right)
$$

with inverse $\phi^{-1}:\left(x_{1}: x_{2}: x_{3}: x_{4}\right) \mapsto\left(x_{1}: x_{2}: x_{3}\right)$. This isomorphism identifies the points $\left\{l_{1}=l_{2}=0\right\} \in C_{3}(K)$ and $(0: 0: 0: 1) \in C_{4}(K)$. To prove the equivalence of (i) and (ii) we note that if $C_{3} \subset \mathbb{P}^{2}$ meets some line in the divisor $D=P_{1}+P_{2}+P_{3}$ then the points $\phi\left(P_{1}\right), \phi\left(P_{2}\right), \phi\left(P_{3}\right)$ and $(0: 0: 0: 1)$ are coplanar on $C_{4} \subset \mathbb{P}^{3}$. 
A generic computation shows that the genus-one models (3-4) and (3-5) in Lemma 3.14 have the same discriminant. Likewise the models (3-6) and (3-7) in Lemma 3.15 have the same discriminant.

Proof of Lemma 3.9. (i) Let $D \in \operatorname{Div}_{K}(E)$ be a divisor of degree 2, and suppose the pair $(E,[D])$ is represented by an integral generalised binary quartic of discriminant $\Delta$. By Lemma 3.13 (with $n=2$ ) we may assume that $P$ is the point $\left(x_{1}: x_{2}: y\right)=(1: 0: \eta)$ for some $\eta \in K$. Since $\mathfrak{O}_{K}$ is integrally closed it follows that $\eta \in \mathfrak{O}_{K}$. By making a substitution $y \leftarrow y+\eta x_{1}^{2}$ we may assume that $\eta=0$. Our model is now of the form (3-4) with $f_{1}\left(x_{1}, x_{2}\right)=x_{2}$. Then the ternary cubic (3-5) is an integral model of discriminant $\Delta$ representing the pair $(E,[D+P])$.

(ii) Let $D \in \operatorname{Div}_{K}(E)$ be a divisor of degree 3, and suppose the pair $(E,[D])$ is represented by an integral ternary cubic of discriminant $\Delta$. By Lemma 3.13 (with $n=3)$ we may assume that $P$ is the point $\left(x_{1}: x_{2}: x_{3}\right)=(0: 0: 1)$. Our model is now of the form (3-6) with $l_{1}=x_{1}$ and $l_{2}=x_{2}$. We may choose the quadratic forms $q_{1}$ and $q_{2}$ to have coefficients in $\mathscr{O}_{K}$. Then the quadric intersection (3-7) is an integral model of discriminant $\Delta$ representing the pair $(E,[D+P])$.

Proof of Lemma 3.10. (i) Let $D \in \operatorname{Div}_{K}(E)$ be a divisor of degree 3, and suppose the pair $(E,[D])$ is represented by an integral ternary cubic of discriminant $\Delta$. By Lemma 3.13 (with $n=3$ ) we may assume that $P$ is the point $(X: Y: Z)=(0: 1: 0)$. Our model is now of the form (3-5). Then the generalised binary quartic (3-4) is an integral model of discriminant $\Delta$ representing the pair $(E,[D-P])$.

(ii) Let $D \in \operatorname{Div}_{K}(E)$ be a divisor of degree 4, and suppose the pair $(E,[D])$ is represented by an integral quadric intersection of discriminant $\Delta$. By Lemma 3.13 (with $n=4$ ) we may assume that $P$ is the point $\left(x_{1}: x_{2}: x_{3}: x_{4}\right)=(0: 0: 0: 1)$. Our model is now of the form (3-7) for some forms $l_{1}, l_{2}, q_{1}$ and $q_{2}$ with coefficients in $\mathbb{O}_{K}$. Then the ternary cubic (3-6) is an integral model of discriminant $\Delta$ representing the pair $(E,[D-P])$.

Remark 3.16. In principle these proofs give an algorithm for minimising $K$-soluble models, but only once a $K$-rational point is explicitly known. (Although it is easy to decide solubility over local fields, if the model is far from minimal then we would need to know a local point to very high precision. Hence our comment that this is not a readily implementable algorithm.)

\section{Minimisation algorithms}

In this section we give algorithms for minimising binary quartics (case $n=2$ ), ternary cubics (case $n=3$ ) and quadric intersections (case $n=4$ ). As in Section 3A we work over a field $K$ which is the field of fractions of a discrete valuation ring $\mathrm{O}_{K}$. There is no need to assume that $K$ is complete (or even Henselian). We 
fix a uniformiser $\pi$ and write $k=\mathbb{O}_{K} / \pi \mathbb{O}_{K}$ for the residue field. In the cases $n=2,4$ we initially assume that $\operatorname{char}(k) \neq 2$, leaving the case $\operatorname{char}(k)=2$ to Section 4D.

Our algorithms for $n=2,3$ share some common features which we now elucidate. In these cases we specify a procedure that takes as input an integral genusone model of positive level, and returns a $K$-equivalent integral model of the same or smaller level. We then show that if the model is nonminimal then the level must decrease after finitely many iterations, and give a bound $N$ on the number of iterations required. This also gives a test for minimality: if $N$ iterations of the procedure fail to decrease the level, then the model must be minimal.

The proofs are by induction on the slope, which we define as the least valuation of the determinant of a matrix $M \in \mathrm{GL}_{n}(K)$ with entries in $\mathrm{O}_{K}$ that can be used to decrease the level. The slope of a minimal model is undefined. The arguments we use are incapable of proving the Minimisation Theorem, since we assume at the outset that the given model has a slope, i.e., is nonminimal.

The following lemma is used to show that our procedure gives a well-defined map on $\mathrm{O}_{K}$-equivalence classes. This is useful, since it means we are free to replace our model by an $O_{K}$-equivalent one at any stage of the proof. We write $I_{m}$ for the $m \times m$ identity matrix.

Lemma 4.1. Let $\mathrm{GL}_{n}(K)$ act on $\mathbb{P}^{n-1}$ in the natural way (via left multiplication of column vectors by matrices). Let $\alpha=\operatorname{Diag}\left(I_{r}, \pi I_{n-r}\right)$ for some $0<r<n$. Then the subgroup of $\mathrm{GL}_{n}\left(\mathrm{O}_{K}\right)$ consisting of transformations whose reduction modulo $\pi$ preserves the subspace $\left\{x_{r+1}=\ldots=x_{n}=0\right\}$ is

$$
\mathrm{GL}_{n}\left(\mathrm{O}_{K}\right) \cap \alpha \mathrm{GL}_{n}\left(\mathrm{O}_{K}\right) \alpha^{-1} \text {. }
$$

Proof. Identifying $\mathbb{P}^{n-1}(K)$ with the nonzero elements of $K^{n}$ modular scalars, $\mathrm{GL}_{n}\left(\mathrm{O}_{K}\right)$ is the subgroup preserving $\mathrm{O}_{K}^{n}$ and we are interested in the subgroup which also preserves $\mathbb{O}_{K}^{r} \oplus\left(\pi \mathscr{O}_{K}\right)^{n-r}=\alpha\left(\mathscr{O}_{K}^{n}\right)$. The statement is now clear.

This lemma is used as follows. Suppose that $\Phi$ and $\Psi$ are $\mathrm{GL}_{n}\left(\mathrm{O}_{K}\right)$-equivalent models, and the matrix relating them (or its transpose, depending on conventions) is one whose reduction modulo $\pi$ preserves the subspace $\left\{x_{r+1}=\ldots=x_{n}=0\right\}$. Then the models $\Phi^{\prime}$ and $\Psi^{\prime}$ obtained by applying $\alpha=\operatorname{Diag}\left(I_{r}, \pi I_{n-r}\right)$ to both $\Phi$ and $\Psi$ will again be $\mathrm{GL}_{n}\left(\mathrm{O}_{K}\right)$-equivalent.

4A. Minimisation of 2-coverings. Let $F \in K[x, z]$ be a binary quartic, say

$$
F(x, z)=a x^{4}+b x^{3} z+c x^{2} z^{2}+d x z^{3}+e z^{4} .
$$

Viewing the set of these as a subset of $X_{2}(K)$, the group of $K$-equivalences between binary quartics is $K^{\times} \times \mathrm{GL}_{2}(K)$, where $[\mu, M]$ acts via $F \mapsto \mu^{2}(F \circ M)$. Note that $\left[\pi^{-2}, \operatorname{Diag}(\pi, \pi)\right]$ acts trivially, so we may if convenient assume that $M$ has entries in $\mathscr{O}_{K}$, not all in $\pi \mathscr{O}_{K}$. 
We say that an integral binary quartic $F$ is minimal if $v(\Delta(F))$ is minimal among all integral binary quartics $K$-equivalent to $F$. If $\operatorname{char}(k)=2$ then this need not be the same as being minimal as a generalised binary quartic. We define the valuation $v(F)$ to be the minimum of the valuations of the coefficients. If $v(F) \geq 2$, then $F$ is not minimal, and indeed dividing through by $\pi^{2}$ gives a $K$-equivalent integral model of smaller level. The algorithm for minimising binary quartics is described in the following theorem.

Theorem 4.2. Let $F \in \mathcal{O}_{K}[x, z]$ be a nonsingular binary quartic. Suppose that $v(F)=0$ or 1 , but $F$ has positive level. If $\operatorname{char}(k)=2$ then further assume that $F$ is nonminimal. Then

(i) The reduction mod $\pi$ of $F_{1}(x, z)=\pi^{-v(F)} F(x, z)$ has either a triple or quadruple root defined over $k$.

(ii) The following procedure replaces $F$ by a K-equivalent integral model of the same level.

- Move the repeated root of $F_{1}(x, z) \bmod \pi$ to $(x: z)=(0: 1)$.

- Replace $F(x, z)$ by $\pi^{-2} F(\pi x, z)$.

(iii) If $F$ is nonminimal then the procedure in (ii) gives $v(F) \geq 2$ after at most 2 iterations.

Proof. We first prove the theorem in the case $F \in \mathfrak{O}_{K}[x, z]$ is nonminimal. By hypothesis there exists $[\mu, M] \in K^{\times} \times \mathrm{GL}_{2}(K)$ with

$$
v(\mu \operatorname{det}(M)) \leq-1 \quad \text { and } \quad v\left(\mu^{2}(F \circ M)\right) \geq 0,
$$

i.e., the transform of $F$ by $[\mu, M]$ has smaller level and is still integral. Hence $v(F \circ M) \geq 2 v(\operatorname{det} M)+2$. The slope $s$ of $F$ is defined to be the least possible valuation of $\operatorname{det} M$, for $M$ such a matrix with entries in $0_{K}$. By Lemma 4.1 we are free to replace $F$ by any $\mathrm{O}_{K}$-equivalent binary quartic. So, putting $M$ in Smith normal form, we may assume that $F\left(\pi^{s} x, z\right) \equiv 0\left(\bmod \pi^{2 s+2}\right)$ where $s$ is the slope. For $s \geq 2$, this condition works out as $\pi^{2}\left|c, \pi^{s+2}\right| d$ and $\pi^{2 s+2} \mid e$. So the only possible slopes are $s=0,1,2$ (as if these conditions hold for some $s>2$, then they also hold for $s=2$, and $s$ was defined to be minimal). If $s=0$, then $v(F) \geq 2$ contrary to hypothesis. If $s=1$, then the coefficients of $F$ have valuations satisfying $\geq 0, \geq 1, \geq 2, \geq 3$ and $\geq 4$. So either $v(F)=0$ and $F(x, z) \bmod \pi$ has a quadruple root at $(x: z)=(0: 1)$, or $v(F)=1$ and $\pi^{-1} F(x, z) \bmod \pi$ has a triple or quadruple root at $(x: z)=(0: 1)$. If $s=2$, then the coefficients of $F$ have valuations satisfying $\geq 0,=0, \geq 2, \geq 4$ and $\geq 6$. Then $F(x, z) \bmod \pi$ has a triple root at ( $0: 1)$. In each of these cases ( $s=1$ and $s=2)$ statements (i) and (ii) of the theorem are now immediate. Moreover the procedure in (ii) returns a $K$-equivalent 
integral model of smaller slope. Hence at most 2 iterations are required to give $v(F) \geq 2$, establishing (iii).

It remains to prove (i) and (ii) in the case $\operatorname{char}(k) \neq 2$ and $F$ has positive level (but could be minimal). Statement (i) follows from the fact that $F_{1} \bmod \pi$ is a null form, i.e., both the invariants $I$ and $J$ vanish. (Since $k$ is perfect the multiple root is defined over $k$.) For (ii) we must show that if $v(F)=0$ and the reduction of $F \bmod \pi$ has a repeated root at $(x: z)=(0: 1)$ then $\pi^{2} \mid e$. But in this case there are smooth $\bar{k}$-points on the reduction of $\mathscr{C} \bmod \pi$ where $\mathscr{C}=\left\{y^{2}=F(x, z)\right\}$. We then have $\mathscr{C}\left(K^{\mathrm{sh}}\right) \neq \varnothing$, and Theorem 3.4 shows that $F$ is nonminimal over $K^{\text {sh }}$. Our earlier argument now applies, keeping in mind that $\pi$ is still a uniformizer for $K^{\text {sh }}$.

To give a satisfactory analogue of this algorithm when $\operatorname{char}(k)=2$ we must work with generalised binary quartics. We give details in Section 4D.

4B. Minimisation of 3-coverings. The valuation $v(F)$ of a ternary cubic $F(x, y, z)=a x^{3}+b y^{3}+c z^{3}+a_{2} x^{2} y+a_{3} x^{2} z+b_{1} x y^{2}+b_{3} y^{2} z+c_{1} x z^{2}+c_{2} y z^{2}+m x y z$ is the minimum valuation of a coefficient. If $v(F) \geq 1$ then $F$ is nonminimal, and indeed dividing through by $\pi$ gives a $K$-equivalent integral model of smaller level. The algorithm for minimising ternary cubics is described in the following theorem.

Theorem 4.3. Let $F \in X_{3}\left(O_{K}\right)$ be a nonsingular ternary cubic. Suppose $v(F)=0$, but $F$ has positive level. Then

(i) The singular locus of the reduction

$$
\mathscr{S}=\left\{(x: y: z) \in \mathbb{P}^{2} \mid F \equiv \frac{\partial F}{\partial x} \equiv \frac{\partial F}{\partial y} \equiv \frac{\partial F}{\partial z} \equiv 0(\bmod \pi)\right\}
$$

is either a point or a line, and is defined over $k$.

(ii) The following procedure replaces $F$ by a K-equivalent integral ternary cubic of the same level.

- Make a $\mathrm{GL}_{3}\left(\mathrm{O}_{K}\right)$-transformation to move the singular locus $\mathscr{S}$ to the point (1:0:0), respectively the line $\{z=0\}$.

- Replace $F(x, y, z)$ by $\pi F\left(\pi^{-1} x, y, z\right)$, respectively $\pi^{-1} F(x, y, \pi z)$.

(iii) If $F$ is nonminimal then the procedure in (ii) gives $v(F) \geq 1$ after at most 4 iterations.

Proof. We are given that $F$ has positive level. It follows that its reduction mod $\pi$ is a null-form, i.e., the invariants $c_{4}, c_{6}$ and $\Delta$ all vanish. The classification of singular ternary cubics (up to equivalence over an algebraically closed field) is well known. See for example [Dolgachev 2003, §10.3] or [Poonen 2001]. The possible null-forms are either a cuspidal cubic, a line touching a conic, three lines 
through a common point, a double line and a line, or a triple line. So over $\bar{k}$ the singular locus of the reduction is either a point or a line. Since $k$ is perfect, this point or line is already defined over $k$. This proves (i).

Next we prove (ii) and (iii) in the case $F$ is nonminimal. By hypothesis there exists $[\mu, M] \in K^{\times} \times \mathrm{GL}_{3}(K)$ with $v(\mu \operatorname{det}(M)) \leq-1$ such that the transform of $F$ by $[\mu, M]$ is still integral. The slope $s$ of $F$ is the least possible valuation of $\operatorname{det} M$, for $M$ such a matrix with entries in $\mathscr{O}_{K}$. By Lemma 4.1 we are free to replace $F$ by any $O_{K}$-equivalent ternary cubic. So putting the transformation matrix in Smith normal form we may assume that $F$ satisfies

$$
F\left(x, \pi^{a} y, \pi^{b} z\right) \equiv 0\left(\bmod \pi^{a+b+1}\right)
$$

for some $0 \leq a \leq b$ with $a+b=s$. If $a=b=0$ then $v(F) \geq 1$, contrary to hypothesis. If $a=0$ and $b \geq 1$ then the reduction of $F \bmod \pi$ only involves the monomials $x z^{2}$, $y z^{2}$ and $z^{3}$. Hence $\mathscr{S}$ is the line $\{z=0\}$. If $a \geq 1$ then the coefficients of $x^{3}, x^{2} y$ and $x^{2} z$ all vanish $\bmod \pi$. Hence $\mathscr{Y}$ is either the point $(1: 0: 0)$ or a line through this point. In each of these cases it is clear that the procedure in (ii) returns an integral model of the same level and smaller slope. Moreover it gives $v(F) \geq 1$ after a finite number of iterations (bounded by the initial slope). The next lemma shows that the only possible slopes are $0,1,2,3$ and 5 . Hence at most 4 iterations are required, establishing (iii).

It remains to prove (ii) in the case $F$ has positive level, but could be minimal. We must show that if $(1: 0: 0)$ is the only singular point on the reduction then $F(1,0,0) \equiv 0\left(\bmod \pi^{2}\right)$. But in this case there are smooth $\bar{k}$-points on the reduction. The proof is completed exactly as in Theorem 4.2.

We say that a pair $(a, b)$ is admissible for $F$ if (4-1) holds.

Lemma 4.4. If some pair $(a, b)$ with $0 \leq a \leq b$ is admissible for $F$ then at least one of the pairs $(0,0),(0,1),(1,1),(1,2)$ or $(2,3)$ is admissible for $F$.

Proof. Suppose $(a, b)$ is admissible for $F$. We make the observations:

- If $a=0$ and $b \geq 1$ then $(0,1)$ is admissible.

- If $a=b \geq 1$ then $(1,1)$ is admissible.

- If $a \geq 1$ and $b \geq 2 a$ then $(1,2)$ is admissible.

- If $a \geq 2$ and $b \geq a+1$ then $(2,3)$ is admissible.

The only remaining possibility is $(a, b)=(0,0)$.

Example 4.5. We apply our algorithm to a cuspidal cubic (although this is singular, there are $\pi$-adically close smooth ternary cubics that are treated in the same way by our algorithm). An arrow labelled $(0, a, b)$ indicates that we make the 
transformation $\left[\pi^{-a-b}, \operatorname{Diag}\left(1, \pi^{a}, \pi^{b}\right)\right]$.

$$
\begin{aligned}
x z^{2}-y^{3} & \stackrel{(0,1,1)}{\longrightarrow} x z^{2}-\pi y^{3} \\
& \stackrel{(0,0,1)}{\longrightarrow} \pi x z^{2}-y^{3} \\
& \stackrel{(0,1,0)}{\longrightarrow} x z^{2}-\pi^{2} y^{3} \\
& \stackrel{(0,0,1)}{\longrightarrow} \pi\left(x z^{2}-y^{3}\right) .
\end{aligned}
$$

So this is an example where our algorithm takes the maximum possible of 4 iterations to give $v(F) \geq 1$.

4C. Minimisation of 4-coverings. In this subsection we prove the Strong Minimisation Theorem, 3.5(i), and Theorem 3.6 in the case $n=4$, assuming that $\operatorname{char}(k) \neq 2$. The proofs are constructive and give an algorithm for minimising quadric intersections. The modifications required when $\operatorname{char}(k)=2$ are described in the next subsection.

We define a map

$$
\mathfrak{d}: X_{4}(K) \rightarrow X_{2}(K), \quad\left(Q_{1}, Q_{2}\right) \mapsto F(x, z)=\operatorname{det}(A x+B z),
$$

where $A$ and $B$ are the matrices of second partial derivatives of $Q_{1}$ and $Q_{2}$. As noted in Definition 2.4 we have $\Delta\left(Q_{1}, Q_{2}\right)=2^{-12} \Delta(F)$.

Lemma 4.6. Let $\left(Q_{1}, Q_{2}\right) \in X_{4}(K)$ be a nonsingular quadric intersection. Then $F=\mathfrak{d}\left(Q_{1}, Q_{2}\right)$ is nonsingular, and there is a morphism of genus-one curves $\mathscr{C}_{\left(Q_{1}, Q_{2}\right)} \rightarrow \mathscr{C}_{F}$ defined over $K$.

Proof. A formula for this morphism is given by classical invariant theory [An et al. 2001; Merriman et al. 1996]. We write the binary quartic $F=\mathfrak{d}\left(Q_{1}, Q_{2}\right)$ as $F(x, z)=a x^{4}+b x^{3} z+c x^{2} z^{2}+d x z^{3}+e z^{4}$, and let $T_{1}$ and $T_{2}$ be the quadrics whose matrices of second partial derivatives $M_{1}$ and $M_{2}$ are determined by

$$
\operatorname{adj}(\operatorname{adj}(A) x+\operatorname{adj}(B) z)=a^{2} A x^{3}+a M_{1} x^{2} z+e M_{2} x z^{2}+e^{2} B z^{3} .
$$

Then $J^{2} \equiv F\left(T_{1},-T_{2}\right) \bmod \left(Q_{1}, Q_{2}\right)$ where $J=\frac{1}{4} \frac{\partial\left(Q_{1}, Q_{2}, T_{1}, T_{2}\right)}{\partial\left(x_{1}, x_{2}, x_{3}, x_{4}\right)}$.

Lemma 4.7. If $[M, N] \in \mathscr{G}_{4}(K)$ then there is a commutative diagram

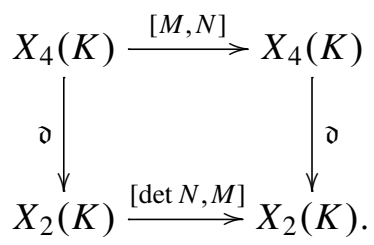

In particular $\mathfrak{d}$ induces a well-defined map on K-equivalence classes.

Proof. This is clear. 
Following [Womack 2003], we deduce the Minimisation Theorem for $n=4$ from the $n=2$ version. The modifications required to prove Theorems 3.5(i) and 3.6 are given at the end of this subsection (see Proposition 4.12).

Proposition 4.8. If $\left(Q_{1}, Q_{2}\right) \in X_{4}(K)$ is nonsingular and $K$-soluble then it is $K$-equivalent to an integral model of level 0.

Proof. Since $\left(Q_{1}, Q_{2}\right)$ is $K$-soluble, it follows by Lemma 4.6 that $\mathfrak{d}\left(Q_{1}, Q_{2}\right)$ is $K$-soluble. So by the Minimisation Theorem for $n=2$ we know that $\mathfrak{d}\left(Q_{1}, Q_{2}\right)$ is $K$-equivalent to an integral binary quartic $F(x, z)$ of level 0 . It is clear by Lemma 4.7 that $\left(Q_{1}, Q_{2}\right)$ is $K$-equivalent to a quadric intersection $\left(Q_{1}^{\prime}, Q_{2}^{\prime}\right)$ with $\mathfrak{d}\left(Q_{1}^{\prime}, Q_{2}^{\prime}\right)=F$. The following lemma shows we may take $\left(Q_{1}^{\prime}, Q_{2}^{\prime}\right)$ integral. This is then the required integral model of level 0 .

Notice that the next three lemmas are false when $\operatorname{char}(k)=2$, as we could otherwise use the above proof to find integral models of level $-v(2)$.

Lemma 4.9. Let $\left(Q_{1}, Q_{2}\right) \in X_{4}(K)$ be a $K$-soluble nonsingular quadric intersection. If $\mathfrak{d}\left(Q_{1}, Q_{2}\right)$ is integral then $\left(Q_{1}, Q_{2}\right)$ is $K$-equivalent to an integral quadric intersection $\left(Q_{1}^{\prime}, Q_{2}^{\prime}\right)$ with $\mathfrak{d}\left(Q_{1}^{\prime}, Q_{2}^{\prime}\right)=\mathfrak{d}\left(Q_{1}, Q_{2}\right)$.

Proof. By a transformation $\left[\mu I_{2}, I_{4}\right]$ for suitable $\mu \in \mathcal{O}_{K}$ we obtain an integral quadric intersection $\left(Q_{1}^{\prime}, Q_{2}^{\prime}\right)$ with $\mathfrak{d}\left(Q_{1}^{\prime}, Q_{2}^{\prime}\right)=\mu^{4} \mathfrak{d}\left(Q_{1}, Q_{2}\right)$. We now apply the following lemma, as many times as required, at each stage preserving the integrality of $\left(Q_{1}^{\prime}, Q_{2}^{\prime}\right)$ while dividing $\mathfrak{d}\left(Q_{1}^{\prime}, Q_{2}^{\prime}\right)$ by a square in $\pi \widehat{O}_{K}$.

Recall that we write $v(F)$ for the minimum of the valuations of the coefficients of the binary quartic $F$. The following is Womack's "main reduction lemma".

Lemma 4.10. Let $\left(Q_{1}, Q_{2}\right) \in X_{4}\left(\mathscr{O}_{K}\right)$ be a nonsingular $K$-soluble integral quadric intersection. If $F=\mathfrak{d}\left(Q_{1}, Q_{2}\right)$ satisfies $v(F) \geq 2$ then $\left(Q_{1}, Q_{2}\right)$ is $K$-equivalent to an integral quadric intersection of smaller level by means of a transformation $\left[\lambda I_{2}, N\right] \in \mathscr{G}_{4}(K)$ with $\lambda \in K^{\times}$and $N \in \mathrm{GL}_{4}(K)$.

The following geometric lemma prepares for the proof of Lemma 4.10. We say that two pairs of quadratic forms in $m$ variables are $k$-equivalent if they are in the same orbit for the natural action of $\mathrm{GL}_{2}(k) \times \mathrm{GL}_{m}(k)$. (This extends our earlier definition in the case $m=4$.) Over an algebraically closed field, the lemma may alternatively be deduced from the classification of pairs of quadrics using the Segre symbol, as given in [Hodge and Pedoe 1952, Chapter XIII, §11].

Lemma 4.11. Let $Q_{1}$ and $Q_{2}$ be quadratic forms in $m=3$ or 4 variables over a field $k$ with $\operatorname{char}(k) \neq 2$. Let $A$ and $B$ be the matrices of second partial derivatives of $Q_{1}$ and $Q_{2}$. Assume that

- $\left\{Q_{1}=Q_{2}=0\right\} \subset \mathbb{P}^{m-1}$ is not a cone, that is, $\operatorname{ker} A \cap \operatorname{ker} B=0$, and

- the binary form $F(x, z)=\operatorname{det}(A x+B z)$ is identically zero. 
Then the k-equivalence class of $\left(Q_{1}, Q_{2}\right)$ is uniquely determined:

(i) If $m=3$ then $\left(Q_{1}, Q_{2}\right)$ is k-equivalent to $\left(x_{1} x_{2}, x_{2} x_{3}\right)$.

(ii) If $m=4$ then $\left(Q_{1}, Q_{2}\right)$ is k-equivalent to $\left(x_{1} x_{2}, x_{2} x_{3}-x_{4}^{2}\right)$.

Proof. (i) We must show that the GCD of $Q_{1}$ and $Q_{2}$ is a linear form, and for this we may assume that $k$ is algebraically closed. Since some quadric in the pencil has rank 2, we may assume that $Q_{1}=x_{1} x_{2}$. Then the condition $\operatorname{det}(A x+B z)=0$ works out as $b_{33}=b_{13} b_{23}=\operatorname{det} B=0$. Swapping $x_{1}$ and $x_{2}$ if necessary, we may assume that $b_{13}=b_{33}=0$. Then $b_{23} \neq 0$ (otherwise we would have a cone) and the condition $\operatorname{det} B=0$ forces $b_{11}=0$. Making a substitution for $x_{3}$ now puts $\left(Q_{1}, Q_{2}\right)$ in the required form.

(ii) Suppose $\left\{Q_{1}=Q_{2}=0\right\} \subset \mathbb{P}^{3}$ has a singular point defined over $k$. Moving this point to $(1: 0: 0: 0)$, it is easy to reduce to the case

$$
A=\left(\begin{array}{cccc}
0 & 1 & 0 & 0 \\
1 & 0 & 0 & 0 \\
0 & 0 & & A^{\prime} \\
0 & 0 &
\end{array}\right), \quad B=\left(\begin{array}{cccc}
0 & 0 & 0 & 0 \\
0 & * & * & * \\
0 & * & & B^{\prime} \\
0 & * &
\end{array}\right)
$$

The condition $\operatorname{det}(A x+B z)=0$ now becomes $\operatorname{det}\left(A^{\prime} x+B^{\prime} z\right)=0$. Hence we may assume that $A^{\prime}$ and $B^{\prime}$ are scalar multiples of

$$
\left(\begin{array}{ll}
0 & 0 \\
0 & 1
\end{array}\right)
$$

Then $b_{23} \neq 0$ (otherwise we have a cone) and a substitution in $x_{3}$ brings us to the case

$$
\left(Q_{1}, Q_{2}\right)=\left(x_{1} x_{2}+\lambda x_{4}^{2}, x_{2} x_{3}+\mu x_{4}^{2}\right)
$$

for some $\lambda, \mu \in k$. Replacing one of these quadrics by a suitable linear combination, and then making a substitution in $x_{1}$ and $x_{3}$ to compensate, we may assume that $\lambda=0$. Then $\mu \neq 0$ (otherwise we have a cone) and we rescale to get $\mu=-1$.

By Theorem 2.8(ii) there is a singular point defined over $\bar{k}$. So running the above proof over $\bar{k}$ shows that $\left\{Q_{1}=Q_{2}=0\right\} \subset \mathbb{P}^{3}$ is the union of a conic and a line, meeting at a unique point. This point of intersection is a $k$-rational singular point. Our earlier proof now applies.

Proof of Lemma 4.10. We write $\bar{Q}_{1}, \bar{Q}_{2}$ for the reductions of $Q_{1}, Q_{2}$ modulo $\pi$. In the proof we often arrive at one of the following three special situations.

Situation 1: The reduction $\mathscr{C}_{\left(\bar{Q}_{1}, \bar{Q}_{2}\right)}$ contains a plane defined over $k$. By means of a $\mathrm{GL}_{4}\left(\mathrm{O}_{K}\right)$-transformation we may move the plane to $\left\{x_{1}=0\right\}$. We apply the transformation $\left[\pi^{-1} I_{2}, \operatorname{Diag}(\pi, 1,1,1)\right]$ to give an integral model of smaller level. 
Situation 2: The reduction $\mathscr{C}_{(}\left(\bar{Q}_{1}, \bar{Q}_{2}\right)$ is a cone over a point $\mathbf{x} \in \mathbb{P}^{3}(k)$, and moreover $Q_{1}(\mathbf{x}) \equiv Q_{2}(\mathbf{x}) \equiv 0\left(\bmod \pi^{2}\right)$. By a $\mathrm{GL}_{4}\left(\mathcal{O}_{K}\right)$-transformation we may move the point to $(1: 0: 0: 0)$. We apply the transformation $\left[I_{2}, \operatorname{Diag}\left(\pi^{-1}, 1,1,1\right)\right]$ to give an integral model of smaller level.

Situation 3: The reduction $\mathscr{C}_{(}\left(\bar{Q}_{1}, \bar{Q}_{2}\right)$ contains a line defined over $k$. By a GL $\mathrm{GL}_{4}\left(\mathrm{O}_{K}\right)$ transformation we may move the line to $\left\{x_{1}=x_{2}=0\right\}$. The flip-flop transformation $\left[\pi^{-1} I_{2}, \operatorname{Diag}(\pi, \pi, 1,1)\right]$ gives an integral model of the same level.

Let $A$ and $B$ be the matrices of second partial derivatives of $Q_{1}$ and $Q_{2}$. Let $\bar{A}$ and $\bar{B}$ be their reductions $\bmod \pi$. We split into cases according to the value of the common nullity, defined as $s=\operatorname{dim}(\operatorname{ker} \bar{A} \cap \operatorname{ker} \bar{B})$.

If $s=0$ then by Lemma 4.11(ii) we are in Situation 3. Applying the flip-flop transformation brings us to the case $s \geq 1$.

If $s=1$ we may assume that $\bar{Q}_{1}$ and $\bar{Q}_{2}$ are quadratic forms in $x_{2}, x_{3}$ and $x_{4}$ only. Let $A^{\prime}$ and $B^{\prime}$ be the 3 by 3 matrices of second partial derivatives. Then

$$
F(x, z) \equiv\left(a_{11} x+b_{11} z\right) \operatorname{det}\left(A^{\prime} x+B^{\prime} z\right)\left(\bmod \pi^{2}\right) .
$$

Since $v(F) \geq 2$ we have either $a_{11} \equiv b_{11} \equiv 0\left(\bmod \pi^{2}\right)$, in which case we are in Situation 2, or $\operatorname{det}\left(A^{\prime} x+B^{\prime} z\right)=0$, in which case Lemma 4.11(i) shows we are in Situation 1 .

If $s \geq 2$ we may assume that $\bar{Q}_{1}$ and $\bar{Q}_{2}$ are binary quadratic forms in $x_{1}$ and $x_{2}$. If $\bar{Q}_{1}$ and $\bar{Q}_{2}$ simultaneously represent 0 over $k$, then we are in Situation 1 . Otherwise we apply the flip-flop transformation $\left[\pi^{-1} I_{2}, \operatorname{Diag}(\pi, \pi, 1,1)\right]$ to give an integral model $\left(R_{1}, R_{2}\right)$ of the same level. Then $\bar{R}_{1}$ and $\bar{R}_{2}$ are binary quadratic forms in $x_{3}$ and $x_{4}$. If $\bar{R}_{1}$ and $\bar{R}_{2}$ simultaneously represent 0 over $k$ then we are in Situation 1. Otherwise we obtain a contradiction to our hypothesis that $\left(Q_{1}, Q_{2}\right)$ is $K$-soluble. Indeed if $\left(x_{1}: x_{2}: x_{3}: x_{4}\right)$ were a $K$-point with $\min \left\{v\left(x_{i}\right): 1 \leq i \leq 4\right\}=0$ then from $Q_{1}(\mathbf{x}) \equiv Q_{2}(\mathbf{x}) \equiv 0(\bmod \pi)$ we deduce $x_{1} \equiv x_{2} \equiv 0(\bmod \pi)$ and from $Q_{1}(\mathbf{x}) \equiv Q_{2}(\mathbf{x}) \equiv 0\left(\bmod \pi^{2}\right)$ we deduce $x_{3} \equiv x_{4} \equiv 0(\bmod \pi)$.

This completes the proof of Proposition 4.8. We now modify the proof so that we can deduce Theorems 3.5(i) and 3.6 in the case $n=4$ from the corresponding results for $n=2$. The situation considered at the end of the last paragraph motivates the definition of a critical model, see Definition 5.1(iii) below.

Proposition 4.12. If $\left(Q_{1}, Q_{2}\right) \in X_{4}(K)$ is nonsingular then it is $K$-equivalent to either an integral model $\Phi \in X_{4}\left(\mathcal{O}_{K}\right)$ with $\mathfrak{d}(\Phi)$ minimal (and hence $\Phi$ minimal), or a critical model (as specified in Definition 5.1(iii), page 793).

Proof. By Lemma 4.7 we may assume that $\mathfrak{d}\left(Q_{1}, Q_{2}\right)$ is a minimal binary quartic. We then follow the proof of Lemma 4.9, but without the hypothesis of $K$-solubility. This hypothesis was only used at the end of the proof of Lemma 4.10. We may 
assume that one of the pairs, say $\bar{Q}_{1}$ and $\bar{Q}_{2}$, simultaneously represents 0 over $\bar{k}$. (Otherwise we would have a critical model.) If they do not simultaneously represent 0 over $k$, then they must be linearly dependent. So it is clear we can reduce the level, but not necessarily using a transformation of the specified form. In the proof of Lemma 4.9 we repeatedly applied Lemma 4.10. For the final application it does not matter what transformation we use. In all earlier applications we have $v(F) \geq 3$. If $A_{1}, B_{1}$ and $A_{2}, B_{2}$ are the 2 by 2 matrices representing the pairs of binary quadratic forms $\bar{Q}_{1}, \bar{Q}_{2}$ and $\bar{R}_{1}, \bar{R}_{2}$ then

$$
F(x, z) \equiv \pi^{2} \operatorname{det}\left(A_{1} x+B_{1} z\right) \operatorname{det}\left(A_{2} x+B_{2} z\right)\left(\bmod \pi^{3}\right) .
$$

The hypothesis $v(F) \geq 3$ therefore ensures that one of the pairs simultaneously represents 0 over $k$. We are then in Situation 1 and can proceed as before.

In Lemma 5.3 (see below) we show that critical models are minimal. Hence following the proof of Proposition 4.12 gives an algorithm for minimising quadric intersections, even in the case they are not $K$-soluble.

Proposition 4.12 also allows us to deduce the case $n=4$ of Theorems 3.5(i) and 3.6 from the case $n=2$. Here we use the easy facts that critical models are $K^{\text {sh }}$-insoluble, and remain critical after any unramified field extension.

4D. Minimisation in residue characteristic 2. We describe how to modify our algorithms in the cases $n=2,4$ when $\operatorname{char}(k)=2$. In the case $n=2$ the issue is that we must work with generalised binary quartics instead of just binary quartics. Recall that a generalised binary quartic, or genus-one model of degree 2 , is an equation of the form

$$
y^{2}+P(x, z) y=Q(x, z),
$$

where $P$ and $Q$ are homogeneous polynomials of degrees 2 and 4 . The coefficients of $P$ and $Q$ are labelled $l, m, n$ and $a, b, c, d, e$. Notice that in characteristic 2 the binary quadratic form $\partial^{2} Q / \partial x \partial z=b x^{2}+d z^{2}$ is a covariant of the quartic $Q$. Moreover this covariant vanishes if and only if $Q$ is a square. (Recall that $k$ is perfect, and so every element of $k$ is a square.)

We say that two models are $y$-equivalent if they are related by a $y$-substitution, that is, a substitution of the form $x \leftarrow x, z \leftarrow z, y \leftarrow y+r_{0} x^{2}+r_{1} x z+r_{2} z^{2}$. The valuation of $(P, Q) \in X_{2}\left(O_{K}\right)$ is

$$
v(P, Q)=\max \left\{\min \left(2 v\left(P^{\prime}\right), v\left(Q^{\prime}\right)\right):\left(P^{\prime}, Q^{\prime}\right) \text { is } y \text {-equivalent to }(P, Q)\right\} .
$$

It is easy to check that $v(P, Q)$ only depends on the $\mathbb{O}_{K}$-equivalence class of $(P, Q)$.

If $v(P)=0$ or $v(P) \geq 1$ and $Q(x, z)$ is not a square $\bmod \pi$ then $v(P, Q)=0$. Otherwise we can make a $y$-substitution so that $v(Q) \geq 1$. Then either $v(Q)=1$, in which case $v(P, Q)=1$, or $v(Q) \geq 2$, in which case $(P, Q)$ is nonminimal, and 
indeed dividing $P$ and $Q$ through by $\pi$ and $\pi^{2}$ gives a $K$-equivalent integral model of smaller level. Theorem 4.2 has the following analogue.

Theorem 4.13. Let $(P, Q) \in X_{2}\left(O_{K}\right)$ be a nonsingular generalised binary quartic. Suppose that $v(P, Q)=0$ or 1 , but $(P, Q)$ has positive level.

(i) The reduction $\bmod \pi$ of

$$
Q_{1}(x, z)= \begin{cases}P(x, z) & \text { if } v(P)=0, \\ \frac{\partial^{2} Q}{\partial x \partial z} & \text { if } v(P) \geq 1 \text { and } v(P, Q)=0, \\ \pi^{-1} Q(x, z) & \text { if } v(P) \geq 1 \text { and } v(Q)=1\end{cases}
$$

has a unique repeated root defined over $k$.

(ii) The following procedure replaces $(P, Q)$ by a $K$-equivalent integral model of the same level.

- If $v(P, Q)=1$ then make a $y$-substitution so that $v(Q) \geq 1$.

- Move the repeated root of $Q_{1}(x, z) \bmod \pi$ to $(x: z)=(0: 1)$.

- Make a y-substitution so that $\pi \mid e$. (This is possible since $\pi \mid n$ and every element of $k$ is a square.)

- Replace $P(x, z)$ by $\pi^{-1} P(\pi x, z)$ and $Q(x, z)$ by $\pi^{-2} Q(\pi x, z)$.

(iii) If $(P, Q)$ is nonminimal then the procedure in (ii) gives $v(P, Q) \geq 2$ after at most 2 iterations.

Proof. We first show that if (i) holds for $(P, Q)$ then it holds for any $\mathrm{O}_{K}$-equivalent model $\left(P^{\prime}, Q^{\prime}\right)$. We say that forms $f, g \in k[x, z]$ are $k$-equivalent if

$$
f(x, z)=\lambda g(\alpha x+\beta z, \gamma x+\delta z)
$$

for some $\lambda, \alpha, \beta, \gamma, \delta \in k$ with $\lambda(\alpha \delta-\beta \gamma) \neq 0$. Each of the following claims is an easy consequence of the definition of $\mathbb{O}_{K}$-equivalence (as given in Section 2) and our assumption that $\operatorname{char}(k)=2$.

- The reductions mod $\pi$ of $P(x, z)$ and $P^{\prime}(x, z)$ are $k$-equivalent; in particular, $v(P)=0 \Longleftrightarrow v\left(P^{\prime}\right)=0$.

- If $v(P) \geq 1$ then the reductions $\bmod \pi$ of $\partial^{2} Q / \partial x \partial z$ and $\partial^{2} Q^{\prime} / \partial x \partial z$ are $k$-equivalent; note that $v(P, Q)=v\left(P^{\prime}, Q^{\prime}\right)$.

- If $v(P) \geq 1$ and $v(Q)=v\left(Q^{\prime}\right)=1$ then the reductions mod $\pi$ of $\pi^{-1} Q(x, z)$ and $\pi^{-1} Q^{\prime}(x, z)$ are $k$-equivalent.

It is now clear that if (i) holds for $(P, Q)$ then it holds for $\left(P^{\prime}, Q^{\prime}\right)$. Next we show that the procedure in (ii) gives a well-defined map on $\hat{O}_{K}$-equivalence classes. This does not automatically follow from Lemma 4.1, as we must also consider $y$-substitutions. Suppose we start with some model satisfying (i), and carry out the 
first three steps of the procedure in (ii) in two different ways. The result is a pair of $\mathscr{O}_{K}$-equivalent models $(P, Q)$ and $\left(P^{\prime}, Q^{\prime}\right)$ related by some $[1, r, M] \in \mathscr{G}_{2}\left(\mathcal{O}_{K}\right)$. Since the reduction of $M \bmod \pi$ fixes the repeated root $(0: 1)$ we have $\pi \mid m_{21}$. Labelling the coefficients of $(P, Q)$ in the usual way, and likewise for $\left(P^{\prime}, Q^{\prime}\right)$, we have $\pi \mid n, e$ and $\pi \mid n^{\prime}, e^{\prime}$. Therefore $\pi \mid r_{2}$. It is now routine to check that if (ii) holds for $(P, Q)$, i.e. $\pi \mid n, d$ and $\pi^{2} \mid e$, then (ii) holds for $\left(P^{\prime}, Q^{\prime}\right)$, i.e. $\pi \mid n^{\prime}, d^{\prime}$ and $\pi^{2} \mid e^{\prime}$. Moreover the transformed models are related by

$$
\left[1,\left(\pi r_{0}, r_{1}, \pi^{-1} r_{2}\right), \operatorname{Diag}(\pi, 1) M \operatorname{Diag}\left(\pi^{-1}, 1\right)\right] \in \mathscr{G}_{2}\left(\mathscr{O}_{K}\right) .
$$

Thus the procedure gives a well-defined map on $\mathfrak{O}_{K}$-equivalence classes.

We are now free in the proof to replace $(P, Q)$ by any $0_{K}$-equivalent model. So if $(P, Q)$ is nonminimal we may assume that $P\left(\pi^{s} x, z\right) \equiv 0\left(\bmod \pi^{s+1}\right)$ and $Q\left(\pi^{s} x, z\right) \equiv 0\left(\bmod \pi^{2 s+2}\right)$ for some integer $s \geq 0$. We call the least such integer $s$ the slope. As happened for binary quartics, the only possible slopes are $s=0,1,2$. If $s=0$ then $v(P, Q) \geq 2$ contrary to hypothesis. If $s=1$ then the coefficients of $(P, Q)$ have valuations satisfying

$$
\geq 0, \quad \geq 1, \quad \geq 2 ; \quad \geq 0, \quad \geq 1, \quad \geq 2, \quad \geq 3, \quad \geq 4
$$

If $v(P)=0$ then $P(x, z)$ mod $\pi$ has a double root at $(x: z)=(0: 1)$. Otherwise, since every element of $k$ is a square (recall $k$ is perfect), we can make a $y$-substitution $y \leftarrow y+r_{0} x^{2}$ so that $v(Q) \geq 1$. Then $\pi^{-1} Q(x, z) \bmod \pi$ has either a triple or quadruple root at $(x: z)=(0: 1)$. If $s=2$ then the coefficients of $(P, Q)$ have valuations satisfying

$$
\geq 0, \quad \geq 1, \quad \geq 3 ; \quad \geq 0, \quad=0, \quad \geq 2, \quad \geq 4, \quad \geq 6
$$

So in this case $v(P, Q)=0$. If $v(P)=0$ then $P(x, z) \bmod \pi$ has a double root at $(x: z)=(0: 1)$. Otherwise $b x^{2}+d z^{2} \bmod \pi$ has a double root at $(x: z)=(0: 1)$. In each of the cases $s=1,2$ it is now clear that the procedure in (ii) returns a $K$ equivalent integral model of smaller slope. Hence at most 2 iterations are required to give $v(P, Q) \geq 2$.

It remains to prove (i) and (ii) in the case $(P, Q)$ has positive level (but could be minimal). If $(P, Q)$ is $K^{\text {sh }}$-soluble then after an unramified extension we have $\mathscr{C}_{(P, Q)}(K) \neq \varnothing$. Then Theorem 3.4 shows that $(P, Q)$ is non-minimal, and our earlier argument applies. Otherwise, we show in Proposition 5.6 below, that $(P, Q)$ is $\mathrm{O}_{K}$-equivalent to a model whose coefficients have valuations satisfying

$$
\geq 1, \quad \geq 1, \quad \geq 2 ; \quad=1, \quad \geq 2, \quad \geq 2, \quad \geq 3, \quad=3 .
$$

Statements (i) and (ii) are then clear. 
Next we modify the algorithm for minimising quadric intersections, as presented in Section 4C. First we replace $\mathfrak{d}$ by the map

$$
\begin{aligned}
\mathfrak{d}^{\prime}: X_{4}(K) & \rightarrow X_{2}(K), \\
\left(Q_{1}, Q_{2}\right) & \mapsto(P, Q)=\left(\operatorname{pf}\left(x Q_{1}+z Q_{2}\right), \operatorname{rd}\left(x Q_{1}+z Q_{2}\right)\right),
\end{aligned}
$$

where pf and rd were defined in the proof of Lemma 2.9. Then

$$
\Delta\left(Q_{1}, Q_{2}\right)=\Delta(P, Q) .
$$

We call $(P, Q)$ the doubling of $\left(Q_{1}, Q_{2}\right)$. (The reason for this name is that $\mathfrak{d}^{\prime}$ acts as multiplication-by-2 on the Weil-Châtelet group.) The analogue of Lemma 4.6 (using $\mathfrak{d}^{\prime}$ instead of $\mathfrak{d}$ ) is immediate if $\operatorname{char}(K) \neq 2$. Indeed, the covering map $\mathscr{C}_{\left(Q_{1}, Q_{2}\right)} \rightarrow \mathscr{C}_{(P, Q)}$ is given by

$$
\left(x_{1}: x_{2}: x_{3}: x_{4}\right) \mapsto\left(T_{1}:-T_{2}: J^{\prime}\right),
$$

where $J^{\prime}=\frac{1}{2}\left(J-l T_{1}^{2}+m T_{1} T_{2}-n T_{2}^{2}\right)$, and $l, m, n$ are the coefficients of $P$. If $\operatorname{char}(K)=2$, then the role of $J^{\prime}$ is taken by

$$
\begin{aligned}
J^{\prime \prime}=\frac{1}{2}\left(J-l T_{1}^{2}+m T_{1} T_{2}-n T_{2}^{2}+\right. & m n\left(l T_{1}+m T_{2}\right) Q_{1}+\operatorname{lm}\left(n T_{2}+m T_{1}\right) Q_{2} \\
& \left.+l^{2} n^{3} Q_{1}^{2}+\operatorname{lm} n\left(\ln +m^{2}\right) Q_{1} Q_{2}+l^{3} n^{2} Q_{2}^{2}\right) .
\end{aligned}
$$

It may be verified by direct calculation that $T_{1}, T_{2}$ and $J^{\prime \prime}$ have coefficients in

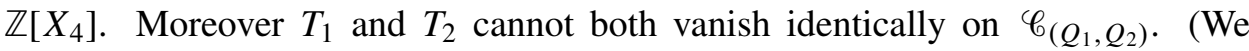
checked this for the models specified in Lemma 3.11, and then used the covariance of $T_{1}$ and $T_{2}$.) Hence in all characteristics there is a morphism $\mathscr{C}_{\left(Q_{1}, Q_{2}\right)} \rightarrow \mathscr{C}_{(P, Q)}$ given by $\left(x_{1}: x_{2}: x_{3}: x_{4}\right) \mapsto\left(T_{1}:-T_{2}: J^{\prime \prime}\right)$.

The diagram in Lemma 4.7 (using $\mathfrak{d}^{\prime}$ instead of $\mathfrak{d}$ ) no longer commutes, but it does commute up to $y$-equivalence, and this is sufficient for our purposes.

Definition 4.14. Let $Q \in k\left[x_{1}, \ldots, x_{m}\right]$ be a quadratic form in $m$ variables.

(i) The kernel $\operatorname{ker} Q$ of $Q$ is the subspace of $k^{m}$ defined by the vanishing of $Q$ and all its partial derivatives. (Recall that $k$ is perfect, so the restriction of $Q$ to the subspace where all the partial derivatives vanish is the square of a linear form.) The rank of $Q$ is $m-\operatorname{dim} \operatorname{ker} Q$.

(ii) The discriminant of $Q$ is

$$
\Delta_{m}(Q)= \begin{cases}\operatorname{det}\left(\partial^{2} Q / \partial x_{i} \partial x_{j}\right) & \text { if } m \text { is even, } \\ \frac{1}{2} \operatorname{det}\left(\partial^{2} Q / \partial x_{i} \partial x_{j}\right) & \text { if } m \text { is odd. }\end{cases}
$$

The discriminant $\Delta_{m}$ is a polynomial in the coefficients of $Q$ with integer coefficients. Therefore Definition 4.14(ii) is valid in all characteristics. Recall that we defined $\mathrm{pf}$ and $\mathrm{rd}$ so that $\Delta_{4}(Q)=\operatorname{pf}(Q)^{2}+4 \operatorname{rd}(Q)$. 
Lemma 4.15. Let $Q_{1}$ and $Q_{2}$ be quadratic forms in $m=3$ or 4 variables over a field $k$ with $\operatorname{char}(k)=2$. Assume that

- $\left\{Q_{1}=Q_{2}=0\right\} \subset \mathbb{P}^{m-1}$ is not a cone, i.e., $\operatorname{ker} Q_{1} \cap \operatorname{ker} Q_{2}=0$, and

- if $m=3$ then $\Delta_{3}\left(x Q_{1}+z Q_{2}\right)=0$, whereas if $m=4$ then $\operatorname{pf}\left(x Q_{1}+z Q_{2}\right)=0$ and $\operatorname{rd}\left(x Q_{1}+z Q_{2}\right)$ is a square.

Then the k-equivalence class of $\left(Q_{1}, Q_{2}\right)$ is uniquely determined, and is as given in Lemma 4.11.

Proof. This is similar to the proof of Lemma 4.11.

In Lemma 4.10 we made the hypothesis that $v(F) \geq 2$ where $F=\mathfrak{d}\left(Q_{1}, Q_{2}\right)$. This should now be replaced by the hypothesis that $\mathfrak{d}^{\prime}\left(Q_{1}, Q_{2}\right)$ is $y$-equivalent to a model $(P, Q)$ with $v(P) \geq 1$ and $v(Q) \geq 2$. Then

$$
\begin{aligned}
& P(x, z)=\operatorname{pf}\left(x Q_{1}+z Q_{2}\right)+2 h(x, z), \\
& Q(x, z)=\operatorname{rd}\left(x Q_{1}+z Q_{2}\right)-\operatorname{pf}\left(x Q_{1}+z Q_{2}\right) h(x, z)-h(x, z)^{2},
\end{aligned}
$$

for some $h \in K[x, z]$. Since $\left(Q_{1}, Q_{2}\right)$ is integral it follows that $h \in \mathscr{O}_{K}[x, z]$. Then $\operatorname{pf}\left(x \bar{Q}_{1}+z \bar{Q}_{2}\right)=0$ and $\operatorname{rd}\left(x \bar{Q}_{1}+z \bar{Q}_{2}\right)$ is a square. Moreover if $\operatorname{rd}\left(x Q_{1}+z Q_{2}\right)$ vanishes $\bmod \pi$ then it vanishes $\bmod \pi^{2}$.

The common nullity is $s=\operatorname{dim}\left(\operatorname{ker} \bar{Q}_{1} \cap \operatorname{ker} \bar{Q}_{2}\right)$. In the case $s=1$ we may assume that $Q_{1}$ and $Q_{2}$ reduce to quadratic forms in $x_{2}, x_{3}$ and $x_{4}$ only. Call these $Q_{1}^{\prime}$ and $Q_{2}^{\prime}$. The analogue of (4-4) is

$$
\operatorname{rd}\left(x Q_{1}+z Q_{2}\right) \equiv(\alpha x+\beta z) \Delta_{3}\left(x Q_{1}^{\prime}+z Q_{2}^{\prime}\right)\left(\bmod \pi^{2}\right)
$$

where $\alpha$ and $\beta$ are the coefficients of $x_{1}^{2}$ in $Q_{1}$ and $Q_{2}$. In all other respects, the proof of the Lemma 4.10 goes through as before. By repeated application of this lemma we obtain the following analogue of Lemma 4.9.

Lemma 4.16. Let $\left(Q_{1}, Q_{2}\right) \in X_{4}(K)$ be a $K$-soluble nonsingular quadric intersection. If $\mathfrak{d}^{\prime}\left(Q_{1}, Q_{2}\right)$ is y-equivalent to an integral generalised binary quartic then $\left(Q_{1}, Q_{2}\right)$ is $K$-equivalent to an integral quadric intersection $\left(Q_{1}^{\prime}, Q_{2}^{\prime}\right)$ such that $\mathfrak{d}^{\prime}\left(Q_{1}^{\prime}, Q_{2}^{\prime}\right)$ is y-equivalent to $\mathfrak{d}^{\prime}\left(Q_{1}, Q_{2}\right)$.

The Minimisation Theorem for $n=4$ now follows from the Minimisation Theorem for $n=2$ exactly as before.

The proof of Proposition 4.12 (with $\mathfrak{d}$ replaced by $\mathfrak{d}^{\prime}$ ) is modified as follows. We follow the proof of Lemma 4.16 but without the hypothesis of $K$-solubility. This hypothesis is only used when $s \geq 2$. In this case

$$
\left(\bar{Q}_{1}, \bar{Q}_{2}\right)=\left(\alpha_{11} x_{1}^{2}+\alpha_{12} x_{1} x_{2}+\alpha_{22} x_{2}^{2}, \beta_{11} x_{1}^{2}+\beta_{12} x_{1} x_{2}+\beta_{22} x_{2}^{2}\right)
$$


and applying the transformation $\left[\pi^{-1} I_{2}, \operatorname{Diag}(\pi, \pi, 1,1)\right]$ gives $\left(R_{1}, R_{2}\right)$ with

$$
\left(\bar{R}_{1}, \bar{R}_{2}\right)=\left(\gamma_{33} x_{3}^{2}+\gamma_{34} x_{3} x_{4}+\gamma_{44} x_{4}^{2}, \delta_{33} x_{3}^{2}+\delta_{34} x_{3} x_{4}+\delta_{44} x_{4}^{2}\right) .
$$

We must show that if $\bar{Q}_{1}$ and $\bar{Q}_{2}$ are linearly dependent and $\mathfrak{d}^{\prime}\left(Q_{1}, Q_{2}\right)$ is $y$ equivalent to a model $(P, Q)$ with $v(P) \geq 2$ and $v(Q) \geq 3$ then one of the pairs $\bar{Q}_{1}, \bar{Q}_{2}$ or $\bar{R}_{1}, \bar{R}_{2}$ simultaneously represents 0 over $k$. Since $s \geq 2$ we already know that $\operatorname{pf}\left(x Q_{1}+z Q_{2}\right)$ vanishes $\bmod \pi$ and $\operatorname{rd}\left(x Q_{1}+z Q_{2}\right)$ vanishes $\bmod \pi^{2}$. It follows by (4-6) that $\operatorname{pf}\left(x Q_{1}+z Q_{2}\right)$ vanishes mod $\pi^{2}$ and $\pi^{-2} \operatorname{rd}\left(x Q_{1}+z Q_{2}\right)$ is a square $\bmod \pi$. Hence

$$
\alpha_{12} \gamma_{34}=\beta_{12} \delta_{34}=\alpha_{12} \delta_{34}+\beta_{12} \gamma_{34}=0
$$

and

$$
\begin{aligned}
& \alpha_{12}^{2}\left(\gamma_{33} \delta_{44}+\gamma_{44} \delta_{33}\right)+\gamma_{34}^{2}\left(\alpha_{11} \beta_{22}+\alpha_{22} \beta_{11}\right)=0 \\
& \beta_{12}^{2}\left(\gamma_{33} \delta_{44}+\gamma_{44} \delta_{33}\right)+\delta_{34}^{2}\left(\alpha_{11} \beta_{22}+\alpha_{22} \beta_{11}\right)=0 .
\end{aligned}
$$

Since $\bar{Q}_{1}$ and $\bar{Q}_{2}$ are linearly dependent we have $\alpha_{11} \beta_{22}+\alpha_{22} \beta_{11}=0$. So either $\alpha_{12}=\beta_{12}=0$, in which case $\bar{Q}_{1}$ and $\bar{Q}_{2}$ simultaneously represent 0 over $k$, or $\gamma_{34}=\delta_{34}=\gamma_{33} \delta_{44}+\gamma_{44} \delta_{33}=0$ in which case $\bar{R}_{1}$ and $\bar{R}_{2}$ simultaneously represent 0 over $k$.

4E. Minimisation over global fields. Our theorems and algorithms for minimisation, as given above, were stated for genus-one models defined over a local field. We now discuss the global situation, and in particular prove Theorem 1.1. We restate that here in a more precise form. A genus-one model defined over a number field is called integral if its coefficients are algebraic integers.

Theorem 4.17. Let $n=2,3$ or 4 . Let $K$ be a number field of class number one. Let $\Phi$ be a nonsingular genus-one model of degree $n$ defined over $K$, and let $E$ be the Jacobian of $\mathscr{C}_{\Phi}$. Suppose that $\mathfrak{C}_{\Phi}$ is locally soluble at all finite places. Then there is a K-equivalent integral genus-one model with discriminant equal to the discriminant of a global minimal Weierstrass equation for $E$.

Proof. To deduce this result directly from the statement of the Minimisation Theorem, 3.4, one would be led to using a version of strong approximation. See [Fisher 2007] for details in the cases $n=2,3$. The case $n=4$ is similar. Although these proofs are not difficult, it is a notable advantage of the algorithmic approach taken in this section that the passage from local to global becomes a triviality.

Indeed, suppose $K$ is a number field with class number one. Let $\mathfrak{p}$ be a prime of $K$ and put $k=O_{K} / \mathfrak{p}$. Then for any pair of $m$-dimensional subspaces $U, V \subset k^{n}$ there exists $M \in \mathrm{SL}_{n}\left(O_{K}\right)$ whose reduction mod $\mathfrak{p}$ takes $U$ to $V$. (Indeed, the case $\operatorname{dim} U=\operatorname{dim} V=1$ is Lemma 3.13, and the general case is similar.) Since $\mathfrak{p}$ is 
principal, say $\mathfrak{p}=\pi \mathfrak{0}_{K}$, we can therefore follow the algorithms for minimising at $\mathfrak{p}$ using $\pi$ as the uniformiser, without changing the level (or integrality) at other primes.

After first scaling the given model to be integral at all primes, we apply this procedure to the finite number of primes at which the resulting model has positive level. This gives an integral model which has level zero at all primes of $K$. By the definition of level, the discriminant of this model is equal to that of any global minimal model for $E$, up to a unit factor. Since this unit must be a 12 th power, a final scaling by a suitable global unit gives the result.

Theorem 1.1 is an immediate corollary, since every $n$-covering which is locally soluble at all places of $K$ can be represented by a genus-one model defined over $K$.

To extend this theorem to a general number field $K$, we may replace integrality by $S$-integrality, where $S$ is a (finite) set of primes generating the class group, so that the ring of $S$-integers is a principal ideal domain. The minimal model may then only be $S$-integral rather than integral. Just as with Weierstrass models for elliptic curves, there may be no global minimal model when the class number is greater than 1 . In practice, we can alternatively find models which are simultaneously minimal at all primes in any given finite set, while being at least integral at all other primes.

Similar results may be deduced from our local results in the case where $K$ is a function field, i.e., a finite extension of $\mathbb{F}_{q}(t)$.

\section{Minimisation of insoluble genus-one models}

We return to working over a discrete valuation field $K$ as specified in Section 3A. In this section we prove the Converse Theorem, 3.5(ii), which shows that the Strong Minimisation Theorem, 3.5(i), is best possible.

Definition 5.1. (i) A generalised binary quartic $(P, Q) \in X_{2}\left(O_{K}\right)$ is critical if the valuations of its coefficients $l, m, n, a, b, c, d$ and $e$ satisfy

$$
\geq 1, \quad \geq 1, \quad \geq 2 ; \quad=1, \quad \geq 2, \quad \geq 2, \quad \geq 3, \quad=3 .
$$

(ii) A ternary cubic $F \in X_{3}\left(\mathrm{O}_{K}\right)$ is critical if the valuations of its coefficients satisfy the inequalities indicated in the following diagram.

$$
\begin{gathered}
z^{3}=2 \\
z^{2} y z^{2} \\
x^{2} z x y z y^{2} z \quad \geq 2 \quad \geq 1^{\geq 2} \geq 2=1
\end{gathered}
$$

(iii) A quadric intersection $\left(Q_{1}, Q_{2}\right) \in X_{4}\left(\mathbb{O}_{K}\right)$ is critical if the reductions of $Q_{1}$ and $Q_{2} \bmod \pi$ are quadratic forms in $x_{1}$ and $x_{2}$ with no common root in $\mathbb{P}^{1}(\bar{k})$, 
and on putting

$$
\left(R_{1}, R_{2}\right)=\left[\pi^{-1} I_{2}, \operatorname{Diag}(\pi, \pi, 1,1)\right]\left(Q_{1}, Q_{2}\right)
$$

the reductions of $R_{1}$ and $R_{2} \bmod \pi$ are quadratic forms in $x_{3}$ and $x_{4}$ with no common root in $\mathbb{P}^{1}(\bar{k})$.

We show in the next three lemmas that critical models are insoluble, minimal and of positive level. We then show (for $n=2,3$ ) that every $K^{\text {sh }}$-insoluble model is $K$-equivalent to a critical model. There is a corresponding result for models of degree $n=4$.

\section{Lemma 5.2. Critical models are insoluble over $K$.}

Proof. We give details in the case $n=2$. Suppose $(x, y, z) \in K^{3}$ is a nonzero solution of $y^{2}+P(x, z) y=Q(x, z)$. Clearing denominators we may assume that $\min \{v(x), v(z)\}=0$. It follows that $y \in \mathbb{O}_{K}$. Then reducing the equation mod $\pi^{i}$ for $i=1,2,3,4$ we successively deduce $\pi|y, \pi| x, \pi^{2} \mid y$ and $\pi \mid z$. In particular $\min \{v(x), v(z)\}>0$. This is the required contradiction. The cases $n=3,4$ are similar.

Since the definition of a critical model is unchanged by an unramified field extension, it follows immediately that critical models are insoluble over $K^{\text {sh }}$.

\section{Lemma 5.3. Critical models are minimal.}

Proof. In the cases $n=2,3$ we give a very quick proof. Indeed, if $\Phi$ were nonminimal, then our algorithms in Sections 4A, 4B and 4D would succeed in reducing the level. But on the contrary, when given a critical model, these algorithms endlessly cycle between two or three $\hat{O}_{K}$-equivalence classes. (Treating the case $n=4$ in the same way would give a circular argument, as the current lemma was cited at the end of Section 4C.)

Alternatively we can imitate the proof of Lemma 5.2. We give details in the case $n=4$. We define

$$
s\left(Q_{1}, Q_{2}\right)=\max \left\{-v(\operatorname{det} M):\left[M, I_{4}\right]\left(Q_{1}, Q_{2}\right) \in X_{4}\left(\mathscr{O}_{K}\right)\right\} .
$$

Suppose $[M, N] \in \mathscr{G}_{4}(K)$ is a transformation taking the critical model $\Phi=\left(Q_{1}, Q_{2}\right)$ to an integral model of smaller level. We may assume that $N$ has entries in $\mathrm{O}_{K}$, not all in $\pi \widehat{O}_{K}$. Let $\xi_{j}\left(x_{1}, \ldots, x_{4}\right)=\sum_{i=1}^{4} n_{i j} x_{i}$. For $i=1,2$ we put

$$
Q_{i} \circ N=Q_{i}\left(\xi_{1}, \ldots, \xi_{4}\right) \in \mathbb{O}_{K}\left[x_{1}, \ldots, x_{4}\right] .
$$

Our hypothesis is that $s\left(Q_{1} \circ N, Q_{2} \circ N\right)>v(\operatorname{det} N)$.

If $v\left(Q_{1} \circ N\right)=0$ then replacing $Q_{2}$ by $Q_{2}+\lambda Q_{1}$ for suitable $\lambda \in O_{K}$ we may assume that $v\left(Q_{2} \circ N\right)>v(\operatorname{det} N)$. To understand this last condition, we put $N$ in Smith normal form. Explicitly we write $N=U \operatorname{Diag}\left(\pi^{a}, \pi^{b}, \pi^{c}, 1\right) V$ 
for some $U, V \in \mathrm{GL}_{4}\left(\mathrm{O}_{K}\right)$ and $a \geq b \geq c \geq 0$. Since $v\left(Q_{2}\right)=0$ we must have $2 a>v(\operatorname{det} N)=a+b+c$ and therefore $a-b+c \geq 1$. It follows that $Q_{2} \circ V \equiv$ $x_{1}\left(\sum_{i=1}^{4} \epsilon_{i} x_{i}\right)\left(\bmod \pi^{2}\right)$ for some $\epsilon_{i} \in \mathbb{O}_{K}$ with $\epsilon_{2} \equiv \epsilon_{3} \equiv \epsilon_{4} \equiv 0(\bmod \pi)$. In other words, $Q_{2} \equiv \mu l_{1} l_{2}\left(\bmod \pi^{2}\right)$ for some $\mu \in \mathbb{O}_{K}$ and linear forms $l_{1}, l_{2}$ in $\mathrm{O}_{K}\left[x_{1}, \ldots, x_{4}\right]$ with $l_{1} \equiv l_{2}(\bmod \pi)$. This contradicts the definition of a critical model (as it would follow that $R_{2}$ vanishes $\bmod \pi$ ). Hence $v\left(Q_{1} \circ N\right) \geq 1$. Similarly $v\left(Q_{2} \circ N\right) \geq 1$. Since $\bar{Q}_{1}$ and $\bar{Q}_{2}$ are binary quadratic forms with no common root we deduce $\xi_{1} \equiv \xi_{2} \equiv 0(\bmod \pi)$. Let $\xi_{i}^{\prime}=\pi^{-1} \xi_{i}$ for $i=1,2$. We put

$$
\left(R_{1}, R_{2}\right)=\left[\pi^{-1} I_{2}, \operatorname{Diag}(\pi, \pi, 1,1)\right]\left(Q_{1}, Q_{2}\right) .
$$

Let $N^{\prime}$ be the matrix with columns the coefficients of $\xi_{3}, \xi_{4}, \xi_{1}^{\prime}$ and $\xi_{2}^{\prime}$. Then $\left(R_{1}, R_{2}\right)$ is a critical model and $s\left(R_{1} \circ N^{\prime}, R_{2} \circ N^{\prime}\right)>v\left(\operatorname{det} N^{\prime}\right)$. Repeating the same arguments we deduce $\xi_{3} \equiv \xi_{4} \equiv 0(\bmod \pi)$. This contradicts our scaling of the matrix $N$.

The next lemma describes the possible levels of a critical model. For this we need to work explicitly with the $a$-invariants defined in the proof of Lemma 2.9. Although $a_{1}, \ldots, a_{6}$ are not invariants (in the sense of Definition 2.7), they are isobaric in the sense that

$$
\begin{aligned}
n & =2, & a_{i} \circ\left[\mu, 0, \operatorname{Diag}\left(\xi_{1}, \xi_{2}\right)\right] & =\left(\mu \xi_{1} \xi_{2}\right)^{i} a_{i}, \\
n & =3, & a_{i} \circ\left[\mu, \operatorname{Diag}\left(\xi_{1}, \xi_{2}, \xi_{3}\right)\right] & =\left(\mu \xi_{1} \xi_{2} \xi_{3}\right)^{i} a_{i}, \\
& n=4, & a_{i} \circ\left[\operatorname{Diag}\left(\mu_{1}, \mu_{2}\right), \operatorname{Diag}\left(\xi_{1}, \xi_{2}, \xi_{3}, \xi_{4}\right)\right] & =\left(\mu_{1} \mu_{2} \xi_{1} \xi_{2} \xi_{3} \xi_{4}\right)^{i} a_{i},
\end{aligned}
$$

for all $i$. (We use the notation for transformations of genus-one models introduced in Section 2.) In the following we write $t^{(m)}$ as a shorthand for $\pi^{-m} t$.

Lemma 5.4. The level of a critical model is at least 1 and equal to 1 if $\operatorname{char}(k) \nmid n$.

Proof. Case $n=2$. By (2-3) we have $\pi^{i} \mid a_{i}$ for all $i$. A convenient way to check this is to note that $\pi^{-3 / 2} P\left(\pi^{1 / 2} x, z\right)$ and $\pi^{-3} Q\left(\pi^{1 / 2} x, z\right)$ have coefficients in $O_{K}\left[\pi^{1 / 2}\right]$, and then to use the isobaric property. It follows that $(P, Q)$ has positive level. Now suppose that $\operatorname{char}(k) \neq 2$ and $(P, Q)$ has level greater than 1. Completing the square we may assume that $l=m=n=0$. Then $a_{1}=a_{3}=0$ and $y^{2}=$ $x^{3}+a_{2}^{(2)} x^{2}+a_{4}^{(4)} x+a_{6}^{(6)}$ is an integral Weierstrass equation of positive level. According to Tate's algorithm the cubic polynomial

$$
x^{3}+a_{2}^{(2)} x^{2}+a_{4}^{(4)} x+a_{6}^{(6)} \equiv\left(x+c^{(2)}\right)\left(x^{2}-4 a^{(1)} e^{(3)}\right)(\bmod \pi)
$$

has a triple root defined over $k$. This contradicts the definition of a critical model. Case $n=3$. By (2-4) we have $\pi^{i} \mid a_{i}$ for all $i$. A convenient way to check this is to note that $\pi^{-2} F\left(\pi^{2 / 3} x, \pi^{1 / 3} y, z\right)$ has coefficients in $\mathbb{O}_{K}\left[\pi^{1 / 3}\right]$, and then to use the 
isobaric property. It follows that $F$ has positive level. Now suppose that $\operatorname{char}(k) \neq 3$ and $F$ has level greater than 1 . Then

$$
y^{2}+a_{1}^{(1)} x y+a_{3}^{(3)} y=x^{3}+a_{2}^{(2)} x^{2}+a_{4}^{(4)} x+a_{6}^{(6)}
$$

is an integral Weierstrass equation of positive level. By (2-4) we find $a_{2}^{(2)} \equiv a_{4}^{(4)} \equiv$ $0(\bmod \pi)$ and

$$
\begin{aligned}
& a_{1}^{(1)} \equiv m^{(1)}(\bmod \pi), \\
& a_{3}^{(3)} \equiv 9 a b^{(1)} c^{(2)}(\bmod \pi), \\
& a_{6}^{(6)} \equiv-27\left(a b^{(1)} c^{(2)}\right)^{2}+a b^{(1)} c^{(2)}\left(m^{(1)}\right)^{3}(\bmod \pi) .
\end{aligned}
$$

So it suffices to show that if there is a Weierstrass equation over $k$ of the form

$$
y^{2}+\alpha x y+9 \beta y=x^{3}+\left(\alpha^{3}-27 \beta\right) \beta
$$

with $c_{4}=\Delta=0$, then $\beta=0$. We have $c_{4}=\alpha\left(\alpha^{3}-216 \beta\right)$ and $\Delta=-\beta\left(\alpha^{3}+27 \beta\right)^{3}$. Since $216+27=3^{5}$ is nonzero in $k$, it follows that $\beta=0$ as required.

Case $n=4$. The quadric intersection $\left[\pi^{-1} I_{2}, \operatorname{Diag}\left(\pi^{1 / 2}, \pi^{1 / 2}, 1,1\right)\right]\left(Q_{1}, Q_{2}\right)$ has coefficients in $\mathscr{O}_{K}\left[\pi^{1 / 2}\right]$. It follows by the isobaric property of the $a$-invariants that $\pi^{i} \mid a_{i}$ for all $i$ and hence that $\left(Q_{1}, Q_{2}\right)$ has positive level. Now suppose that $\operatorname{char}(k) \neq 2$. Then $F=\mathfrak{d}\left(Q_{1}, Q_{2}\right)$ satisfies $F(x, z) \equiv \pi^{2} f_{1}(x, z) f_{2}(x, z)\left(\bmod \pi^{3}\right)$ where $f_{1}, f_{2} \in \mathcal{O}_{K}[x, z]$ are binary quadratic forms, neither having a repeated root $\bmod \pi$. (So their product cannot have a triple or quadruple root.) It follows by Theorem 4.2(i) that $F$ and hence $\left(Q_{1}, Q_{2}\right)$ has level 1.

Example 5.5. The following examples of critical models, all with level 2, show that the hypothesis char $(k) \nmid n$ cannot be removed from Lemma 5.4.

$$
\begin{array}{ll}
K=\mathbb{Q}_{2} & y^{2}=2 x^{4}+24 x^{2} z^{2}+8 z^{4}, \\
K=\mathbb{Q}_{3} & x^{3}+3 y^{3}+9 z^{3}+18 x y z=0, \\
K=\mathbb{Q}_{2} & x_{1}^{2}+2 x_{3}^{2}+4 x_{2} x_{4}=x_{2}^{2}+2 x_{4}^{2}+4 x_{1} x_{3}=0,
\end{array}
$$

To complete the proof of Theorem 3.5(ii) we prove the following. The doubling map $\mathfrak{d}^{\prime}$ was defined in Section 4D. (If $\operatorname{char}(k) \neq 2$ then we can work with $\mathfrak{d}$ instead.)

Proposition 5.6. Let $\Phi \in X_{n}\left(\mathscr{O}_{K}\right)$ be a $K^{\text {sh }}$-insoluble minimal genus-one model.

(i) If $n=2$ or 3 then $\Phi$ is $\mathbb{O}_{K}$-equivalent to a critical model.

(ii) If $n=4$ then $\Phi$ is $K$-equivalent to either a critical model or an integral model $\left(Q_{1}, Q_{2}\right)$ with $\mathfrak{d}^{\prime}\left(Q_{1}, Q_{2}\right)$ critical.

First we need three lemmas. 
Lemma 5.7. Let $k$ be an algebraically closed field. Suppose that either

(a) $\Phi=(P, Q) \in X_{2}(k)$ and $P^{2}+4 Q$ is not identically zero,

(b) $\Phi=(F) \in X_{3}(k)$ is nonzero and is not the cube of a linear form,

(c) $\Phi=\left(Q_{1}, Q_{2}\right) \in X_{4}(k)$ and every quadric in the pencil spanned by $Q_{1}$ and $Q_{2}$ has rank at least 2 .

Then $\mathscr{C}_{\Phi}$ has a smooth $k$-point (on some 1-dimensional component).

Proof. For $n=2,3$ this is clear. In the case $n=4$ we are looking for a transverse point of intersection of $Q_{1}$ and $Q_{2}$, i.e., a point where the Jacobian matrix has rank 2. We prove the result more generally for intersections of two quadrics in $m$ variables. This enables us to reduce to the case $\operatorname{ker} Q_{1} \cap \operatorname{ker} Q_{2}=0$. Now let $P$ be a singular point on the quadric intersection. (If there is no such point there is nothing to prove.) Then moving this point to $(1: 0: \ldots: 0)$ we may assume that $Q_{1}=x_{1} x_{2}+g_{1}\left(x_{2}, \ldots, x_{m}\right)$ and $Q_{2}=g_{2}\left(x_{2}, \ldots, x_{m}\right)$ for some $g_{1}$ and $g_{2}$. Since $\operatorname{rank}\left(Q_{2}\right) \geq 2$ we can pick a smooth point $\left(x_{2}: \ldots: x_{m}\right)$ on $\left\{Q_{2}=0\right\} \subset \mathbb{P}^{m-2}$ with $x_{2} \neq 0$. Then solving the equation $Q_{1}=0$ for $x_{1}$ gives the required transverse point of intersection on $\left\{Q_{1}=Q_{2}=0\right\}$.

Lemma 5.8. Let $\Phi \in X_{n}\left(\mathcal{O}_{K}\right)$ be a $K^{\mathrm{sh}}$-insoluble minimal genus-one model.

(a) If $n=2$ then $\Phi=(P, Q)$ with $v(P, Q)=1$. Moreover if $v(Q)=1$ then the reduction of $\pi^{-1} Q(x, z)$ mod $\pi$ has either two double roots or a quadruple root (over $\bar{k})$.

(b) If $n=3$ then $\Phi$ is a ternary cubic whose reduction $\bmod \pi$ is (a constant times) the cube of a linear form.

(c) If $n=4$ then there is a quadric of rank 1 in the reduced pencil; that is, if $\Phi=\left(Q_{1}, Q_{2}\right)$ then $\operatorname{rank}\left(\lambda \bar{Q}_{1}+\mu \bar{Q}_{2}\right)=1$ for some $(\lambda: \mu) \in \mathbb{P}^{1}(\bar{k})$.

Proof. We recall that $K^{\text {sh }}$ has residue field $\bar{k}$. The idea of the proof is that if $\Phi$ is not of the form listed, then we can use Lemma 5.7 to find a smooth $\bar{k}$-point on the reduction, and use the Henselian property to lift it to a $K^{\text {sh }}$-point, thereby obtaining a contradiction.

A little more needs to be said in the case $n=2$. If $\operatorname{char}(k) \neq 2$ then completing the square gives $v(P) \geq 1$ and Lemma 5.7 shows that $v(Q) \geq 1$. If $\operatorname{char}(k)=2$ then Lemma 5.7 shows that $v(P) \geq 1$. If $Q(x, z) \bmod \pi$ had a simple root over $\bar{k}$ then we could lift to a $K^{\text {sh }}$-point on $\mathscr{C}_{(P, Q)}$ with $y=0$. It follows that $Q(x, z)$ is a square $\bmod \pi$. So by a $y$-substitution we may suppose $v(Q) \geq 1$. In all residue characteristics we now have $v(P) \geq 1$ and $v(Q) \geq 1$. We cannot have $v(Q) \geq 2$ since $(P, Q)$ is minimal. If $\pi^{-1} Q(x, z) \bmod \pi$ had a simple root over $\bar{k}$ then we could lift to a $K^{\text {sh }}$-point on $\mathscr{C}_{(P, Q)}$ with $y=0$. It follows that this polynomial has either two double roots or a quadruple root. 
Lemma 5.9. Suppose $(P, Q),\left(P^{\prime}, Q^{\prime}\right) \in X_{2}\left(\mathrm{O}_{K}\right)$ are $K$-equivalent models of the same level related by a substitution $[\mu, r, M] \in \mathscr{G}_{2}(K)$ where $M \in \mathrm{GL}_{2}(K)$ has Smith normal form $\operatorname{Diag}\left(1, \pi^{s}\right)$. Then $v(\Delta(P, Q)) \geq 2$ s.

Proof. Let $(P, Q)$ have coefficients $l, m, n, a, b, c, d$ and $e$. Replacing our models by $O_{K}$-equivalent ones we may assume $\mu=\pi^{-s}$ and $M=\operatorname{Diag}\left(\pi^{s}, 1\right)$. If we assume for simplicity that $r=0$, then we have $\pi^{s} \mid n, d$ and $\pi^{2 s} \mid e$. Since the discriminant $\Delta \in \mathbb{Z}\left[X_{2}\right]$ belongs to the ideal $\left(n^{2}, n d, d^{2}, e\right)$ it follows that $v(\Delta(P, Q)) \geq 2 s$.

For general $r$ we can write the transformation $\left[\pi^{-s}, r, \operatorname{Diag}\left(\pi^{s}, 1\right)\right]$ either as

$$
y \leftarrow \pi^{s} y+r_{0} x^{2}+r_{1} x z+r_{2} z^{2} \quad \text { followed by } \quad x \leftarrow \pi^{s} x,
$$

or as

$$
x \leftarrow \pi^{s} x \quad \text { followed by } \quad y \leftarrow \pi^{s}\left(y+\pi^{s} r_{0} x^{2}+r_{1} x z+\pi^{-s} r_{2} z^{2}\right) .
$$

Since $Q^{\prime}$ has coefficients in $\mathscr{O}_{K}$ we have

$$
v\left(r_{0}^{2}+r_{0} l-a\right) \geq-2 s \text { and } v\left(r_{2}^{2}+r_{2} n-e\right) \geq 2 s .
$$

Hence $\pi^{s} r_{0}, r_{2} \in \mathfrak{O}_{K}$. So replacing our models by $\mathbb{O}_{K}$-equivalent ones we may assume that $r_{0}=r_{2}=0$. Then the middle coefficient of $Q^{\prime}$ gives $v\left(r_{1}^{2}+r_{1} m-c\right) \geq 0$ and hence $r_{1} \in \mathfrak{O}_{K}$. Once more replacing $(P, Q)$ by an $\mathrm{O}_{K}$-equivalent model we may assume that $r_{0}=r_{1}=r_{2}=0$. Our earlier proof now applies.

Proof of Proposition 5.6. We split into the cases $n=2,3,4$.

Case $n=2$. Applying Lemma 5.8 to $\Phi=(P, Q)$ we may assume that $v(P) \geq 1$, $v(Q)=1$ and $\pi^{-1} Q(x, z)$ mod $\pi$ has either two double roots or a quadruple root.

We first rule out the possibility of two double roots. After an unramified field extension we may assume that these roots are defined over $k$. So without loss of generality $Q(x, z) \equiv \pi x^{2} z^{2}\left(\bmod \pi^{2}\right)$. We replace $P(x, z)$ by $\pi^{-1} P(\pi x, z)$ and $Q(x, z)$ by $\pi^{-2} Q(\pi x, z)$. By Lemma 5.8 we again have $v(P, Q) \geq 1$. We make a substitution $y \leftarrow y+r_{2} z^{2}$ so that $v(P) \geq 1$ and $v(Q) \geq 1$. Now $\pi^{-1} Q(x, z) \bmod \pi$ has a double root at $(x: z)=(1: 0)$. By Lemma 5.8 it has a second double root, say at $(\lambda: 1)$. We make the substitution $x \leftarrow x+\lambda z$. Then $Q(x, z) \equiv \pi x^{2} z^{2}\left(\bmod \pi^{2}\right)$. We can now repeat this process indefinitely. It follows by Lemma 5.9 that $\Delta(P, Q)=0$. This is the required contradiction.

It remains to consider the case of a quadruple root, say $Q(x, z) \equiv \pi x^{4}\left(\bmod \pi^{2}\right)$. Let $l_{1}, m_{1}, n_{1}, a_{1}, b_{1}, c_{1}, d_{1}$ and $e_{1}$ be the coefficients of $P_{1}(x, z)=\pi^{-1} P(\pi x, z)$ and $Q_{1}(x, z)=\pi^{-2} Q(\pi x, z)$. By Lemma 5.8 we can make a substitution

$$
y \leftarrow y+r_{2} z^{2}
$$

so that $\pi$ divides $n_{1}$ and $e_{1}$. Then $\pi^{-1} Q_{1}(x, z) \bmod \pi$ has at least a triple root at 
$(x: z)=(1: 0)$. So by Lemma 5.8 we have $\pi^{2} \mid d_{1}$ and $v\left(e_{1}\right)=1$. The coefficients of $(P, Q)$ now satisfy the definition of a critical model.

Case $n=3$. By Lemma 5.8 our ternary cubic $F$ must reduce mod $\pi$ to the cube of a linear form. So without loss of generality, we have

$$
F=\pi f_{3}(y, z)+\pi f_{2}(y, z) x+\pi f_{1}(y, z) x^{2}+a x^{3} .
$$

with $\pi \nmid a$. Then $F_{1}(x, y, z)=\pi^{-1} F(\pi x, y, z)$ is a minimal ternary cubic and by Lemma 5.8 its reduction $\bmod \pi$ is the cube of a linear form in $y$ and $z$. After a suitable transformation of $y$ and $z$, we may assume that $f_{3}(y, z) \equiv b y^{3}(\bmod \pi)$ with $\pi \nmid b$ (otherwise $F$ would not be minimal). Now

$$
F_{2}(x, y, z)=\pi^{-1} F_{1}(x, \pi y, z)
$$

is again a minimal ternary cubic, and its reduction $\bmod \pi$ is $\left(c^{\prime} x+c z\right) z^{2}$. Again this must be a nonzero cube. So $c^{\prime}=0$ and $c$ is a unit. The coefficients of $F$ now satisfy the definition of a critical model.

Case $n=4$. We divide the proof into the following two lemmas.

Lemma 5.10. Let $\left(Q_{1}, Q_{2}\right) \in X_{4}\left(\mathbb{O}_{K}\right)$ be a $K^{\text {sh }}$-insoluble minimal quadric intersection. Let

$$
s=\operatorname{dim}\left(\operatorname{ker} \bar{Q}_{1} \cap \operatorname{ker} \bar{Q}_{2}\right)
$$

be the common nullity of the reduced pencil.

(i) If $s \leq 1$ then the reduced pencil contains a unique rank 1 quadric, and the following procedure replaces $\left(Q_{1}, Q_{2}\right)$ by a K-equivalent minimal quadric intersection with $s \geq 1$.

- Make a $\mathrm{GL}_{2}\left(\mathrm{O}_{K}\right) \times \mathrm{GL}_{4}\left(\mathbb{O}_{K}\right)$-transformation so that $Q_{2} \equiv x_{1}^{2}(\bmod \pi)$.

- Apply the transformation $\left[\operatorname{Diag}\left(1, \pi^{-1}\right), \operatorname{Diag}(\pi, 1,1,1)\right]$.

(ii) If $s \geq 2$ then $\left(Q_{1}, Q_{2}\right)$ is $\mathrm{O}_{K}$-equivalent to a critical model.

Proof. (i) By Lemma 5.8 there is a rank 1 quadric in the reduced pencil. It is unique (and therefore defined over $k$ ) as we would otherwise have $s \geq 2$. The remaining statements are clear.

(ii) We may assume that $\bar{Q}_{1}$ and $\bar{Q}_{2}$ are binary quadratic forms in $x_{1}$ and $x_{2}$. Since the model is minimal, these forms have no common root in $\mathbb{P}^{1}(\bar{k})$. We put

$$
\left(R_{1}, R_{2}\right)=\left[\pi^{-1} I_{2}, \operatorname{Diag}(\pi, \pi, 1,1)\right]\left(Q_{1}, Q_{2}\right) .
$$

Then $R_{1}$ and $R_{2}$ reduce to binary quadratic forms in $x_{3}$ and $x_{4}$. Again, since the model is minimal, these forms have no common root in $\mathbb{P}^{1}(\bar{k})$. Hence $\left(Q_{1}, Q_{2}\right)$ is critical. 
Lemma 5.11. Let $\Phi \in X_{4}\left(0_{K}\right)$ satisfy the hypotheses of Lemma 5.10 with $s=1$. If the procedure in Lemma 5.10(i) may be iterated indefinitely, then $\Phi$ is $\mathrm{O}_{K}$ equivalent to a quadric intersection $\left(Q_{1}, Q_{2}\right)$ where the valuations of the coefficients of $Q_{1}$ and $Q_{2}$ satisfy the inequalities indicated in the following diagram:

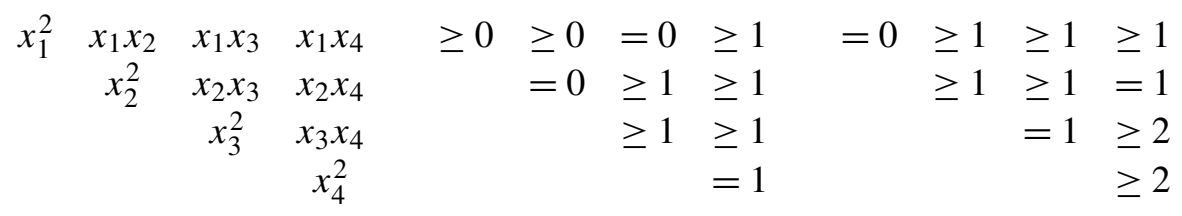

Proof. We may assume that $\Phi=\left(Q_{1}, Q_{2}\right)$ has reduction

$$
\left(\bar{Q}_{1}, \bar{Q}_{2}\right)=\left(x_{1} l\left(x_{2}, x_{3}\right)+f\left(x_{2}, x_{3}\right), c x_{1}^{2}\right)
$$

for some $c \in k$ and $l, f \in k\left[x_{2}, x_{3}\right]$. Since $\left(Q_{1}, Q_{2}\right)$ is minimal we have $c f \neq 0$. So the reduction is (set-theoretically) either a line or a pair of lines. We show in the case of a pair of lines that the procedure in Lemma 5.10(i) must give $s \geq 2$ after a finite number of iterations (bounded in terms of the valuation of the discriminant). The first iteration gives $\left(R_{1}, R_{2}\right)$ with $\left(\bar{R}_{1}, \bar{R}_{2}\right)=\left(f\left(x_{2}, x_{3}\right), g\left(x_{2}, x_{3}, x_{4}\right)\right)$, for some $g \in k\left[x_{2}, x_{3}, x_{4}\right]$. Since $f$ has rank 2 we may assume on replacing $R_{2}$ by $R_{2}+\lambda R_{1}$ for suitable $\lambda \in O_{K}$ that $g$ has rank 1. If $g$ has no coefficient of $x_{4}^{2}$ then $s \geq 2$. Otherwise a $\mathrm{GL}_{4}\left(\mathrm{O}_{K}\right)$-transformation puts $\left(\bar{R}_{1}, \bar{R}_{2}\right)$ in the form (5-1) with $l=0$ (and the same $f$ as before). The process is then repeated. By considering the effect on the doubling it follows by Lemma 5.9 that only finitely many iterations are possible.

It remains to consider the case where the reduction is (set-theoretically) a line. We may assume that $\Phi=\left(Q_{1}, Q_{2}\right)$ and its transforms,

$$
\begin{aligned}
\left(R_{1}, R_{2}\right) & =\left[\operatorname{Diag}\left(1, \pi^{-1}\right), \operatorname{Diag}(\pi, 1,1,1)\right]\left(Q_{1}, Q_{2}\right), \\
\left(S_{1}, S_{2}\right) & =\left[\operatorname{Diag}\left(\pi^{-1}, 1\right), \operatorname{Diag}(1, \pi, 1,1)\right]\left(R_{1}, R_{2}\right),
\end{aligned}
$$

under the first two iterations have reductions

$$
\begin{aligned}
\left(\bar{Q}_{1}, \bar{Q}_{2}\right) & =\left(x_{1}\left(\alpha_{1} x_{1}+\alpha_{2} x_{2}+\alpha_{3} x_{3}\right)+x_{2}^{2}, x_{1}^{2}\right), \\
\left(\bar{R}_{1}, \bar{R}_{2}\right) & =\left(x_{2}^{2}, x_{2}\left(\beta_{2} x_{2}+\beta_{3} x_{3}+\beta_{4} x_{4}\right)+g\left(x_{3}, x_{4}\right)\right), \\
\left(\bar{S}_{1}, \bar{S}_{2}\right) & =\left(\alpha_{3} x_{1} x_{3}+\lambda x_{3}^{3}+\mu x_{3} x_{4}+v x_{4}^{2}, g\left(x_{3}, x_{4}\right)\right),
\end{aligned}
$$

for some $\alpha_{i}, \beta_{i}, \lambda, \mu, v \in k$ and $g \in k\left[x_{3}, x_{4}\right]$. By (5-2) we have $\alpha_{3} \neq 0$ (otherwise $s \geq 2$ ). Since the reduction cannot be a pair of lines, we see first by (5-3) that $g$ has rank 1 , and then by (5-4) that $g=\gamma x_{3}^{2}$ for some $\gamma \neq 0$. Finally (5-3) and (5-4) show that $\beta_{4} \neq 0$ and $v \neq 0$ (otherwise $s \geq 2$ ). The valuations of the coefficients of $Q_{1}$ and $Q_{2}$ now satisfy the inequalities indicated in the statement of the lemma. 
Proposition 5.6(ii) follows from the last two lemmas and the observation that if $\left(Q_{1}, Q_{2}\right)$ satisfies the conclusions of Lemma 5.11 then its doubling is critical.

\section{Reduction}

In this section, we assume that the ground field is $\mathbb{Q}$. The main reason for this is that a comparable theory of reduction over a general number field has not yet been sufficiently developed.

Let $\mathscr{b} \subset \mathbb{P}^{n-1}$ be a genus-one normal curve defined over $\mathbb{Q}$ of degree $n$ (or, if $n=2$, let $\mathscr{b} \rightarrow \mathbb{P}^{1}$ be a double cover) with points everywhere locally, so that $\mathscr{C}$ represents an element of the $n$-Selmer group of its Jacobian elliptic curve $E$. If $n \in\{2,3,4\}$, we can, by the results and algorithms of the previous sections, assume that $\mathscr{b}=\mathscr{C}_{\Phi}$ where $\Phi$ is a genus-one model which is both integral and minimal, so that its invariants $c_{4}, c_{6}$ and $\Delta$ coincide with those of a minimal model of $E$. This means that the invariants are as small as possible (in absolute value). However, it does not necessarily mean that the equations defining $\mathscr{C}$ will have small coefficients. To achieve this, we will employ reduction. Leaving aside the aesthetic value of equations with small coefficients, the main benefit of a reduced model is that further computations like searching for rational points on $\mathscr{C}$ or performing further descents on $\mathscr{b}$ are greatly facilitated.

The idea of reduction is to find a unimodular transformation (i.e., an invertible integral linear change of coordinates on $\mathbb{P}^{n-1}$ ) that makes the equations defining $\mathscr{b}$ smaller. Unimodular transformations have the property of preserving the integrality and invariants of the model, so they will not destroy its minimality. In the language of Section 2, a unimodular transformation is just a $\mathbb{Z}$-equivalence.

If we were allowed to make a coordinate change from $\mathrm{SL}_{n}(\mathbb{C})$ instead, then we could always bring our model into one of the following standard forms, where in general $a, b \in \mathbb{C}$ (see for example [Hulek 1986]). When $n=3$, we can achieve this normal form even by a transformation from $\mathrm{SL}_{3}(\mathbb{R})$, so in this case, we can take $a, b \in \mathbb{R}$. We will call these forms Hesse forms, generalising the classical terminology for $n=3$. They are as follows:

$$
\begin{array}{ll}
n=2: & y^{2}=a\left(x_{0}^{4}+x_{1}^{4}\right)+b x_{0}^{2} x_{1}^{2}, \\
n=3: & a\left(x_{0}^{3}+x_{1}^{3}+x_{2}^{3}\right)+b x_{0} x_{1} x_{2}=0, \\
n=4: & a\left(x_{0}^{2}+x_{2}^{2}\right)+b x_{1} x_{3}=0, \quad a\left(x_{1}^{2}+x_{3}^{2}\right)+b x_{0} x_{2}=0 .
\end{array}
$$

In these forms, the coefficients $a$ and $b$ are bounded in terms of the invariants, so we can expect them to be small. Therefore, we would like to come close to a model of this kind, but using a unimodular transformation. 
We need some way of measuring how close two models are. On the standard Hesse models, the action of the $n$-torsion of the Jacobian, $E[n]$, is given by the socall standard representation, where one generator multiplies each $x_{j}$ by $\zeta_{n}^{j}$ and the other generator does a cyclic shift of the coordinates. (Here $\zeta_{n}$ denotes a primitive $n$-th root of unity.) To this representation, we can associate an invariant inner product on $\mathbb{C}^{n}$, which is unique up to scaling. It is easy to check that this invariant inner product is just the standard one on $\mathbb{C}^{n}$. Now our approach is to associate an inner product to a given model $\mathscr{C}$, and consider the model to be close to a standard model when the associated inner product is close to the standard one, which means that it is reduced in an appropriate sense. This is explained in some detail in the following subsection.

6A. The reduction covariant. Let $K=\mathbb{R}$ or $\mathbb{C}$. We write $\mathscr{Y}_{n}(K)$ for the set of all genus-one normal curves of degree $n$ defined over $K$, inside a fixed copy of $\mathbb{P}^{n-1}$. (If $n=2$ we consider double covers of $\mathbb{P}^{1}$ instead.) The difference between $\mathscr{Y}_{n}(K)$ and $X_{n}(K)$ is that we now consider actual curves in $\mathbb{P}^{n-1}$ (or the set of ramification points of $\mathscr{C} \rightarrow \mathbb{P}^{1}$ when $n=2$ ), instead of defining equations.

Let $\mathcal{H}_{n}^{+}(\mathbb{C})$ be the space of positive definite Hermitian $n \times n$ matrices, and $\mathscr{H}_{n}^{+}(\mathbb{R})$ the space of positive definite symmetric real $n \times n$ matrices. We can identify these spaces with the spaces of positive definite Hermitian and real quadratic forms in $n$ variables, respectively. There are natural and compatible (left) actions of $\mathrm{SL}_{n}(K)$ on $\mathscr{Y}_{n}(K)$ and $\mathscr{H}_{n}^{+}(K)$ given by the canonical map

$$
\mathrm{SL}_{n}(K) \rightarrow \mathrm{PGL}_{n}(K)=\operatorname{Aut}\left(\mathbb{P}_{K}^{n-1}\right)
$$

on the one hand and by

$$
g \cdot M=\bar{g}^{-t} M g^{-1}
$$

on the other, where $\gamma^{-t}$ denotes the inverse transpose of the matrix $\gamma$. If we identify the matrix $M \in \mathscr{H}_{n}^{+}(K)$ with the quadratic or Hermitian form $Q(x)=\bar{x}^{t} M x$, then the compatibility of the actions means that $(g \cdot Q)(g x)=Q(x)$.

Theorem 6.1. For each $n \geq 2$ there is a unique $\mathrm{SL}_{n}(\mathbb{C})$-covariant map

$$
\varphi_{\mathbb{C}}: \mathscr{Y}_{n}(\mathbb{C}) \rightarrow \mathscr{H}_{n}^{+}(\mathbb{C}) / \mathbb{R}_{>0}^{\times} .
$$

This map is compatible with complex conjugation, and so restricts to an $\mathrm{SL}_{n}(\mathbb{R})$ covariant map

$$
\varphi_{\mathbb{R}}: \mathscr{Y}_{n}(\mathbb{R}) \rightarrow \mathscr{H}_{n}^{+}(\mathbb{R}) / \mathbb{R}_{>0}^{\times} .
$$

Proof. Let $\mathscr{b} \rightarrow \mathbb{P}^{n-1}$ be a genus-one normal curve defined over $\mathbb{C}$, with Jacobian $E$. The action of $E[n]$ on $\mathscr{C}$ extends to $\mathbb{P}^{n-1}$ and hence defines a group 
homomorphism $\chi: E[n](\mathbb{C}) \rightarrow \mathrm{PGL}_{n}(\mathbb{C})$. Lifting to $\mathrm{SL}_{n}(\mathbb{C})$ we obtain a diagram

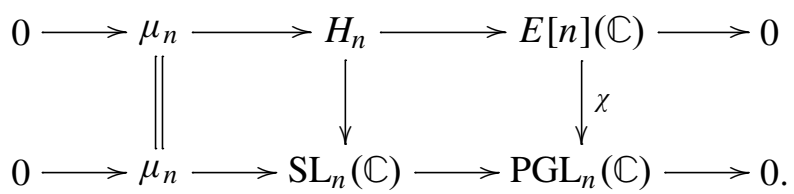

The Heisenberg group $H_{n}$ is a nonabelian group of order $n^{3}$. It comes with a natural $n$-dimensional representation, called the Schrödinger representation, which is known to be irreducible (since it is equivalent to the standard representation mentioned above). Now by the Weyl unitary trick, every irreducible complex representation of a finite group has a unique invariant inner product. (Recall that existence is proved by averaging over the group, and uniqueness (up to $\mathbb{R}_{>0}^{\times}$) using Schur's lemma.)

We define $\varphi_{\mathbb{C}}(\mathscr{C})$ to be the (matrix of the) Heisenberg invariant inner product, i.e., $\varphi_{\mathbb{C}}(\mathscr{C})$ is uniquely determined up to positive real scalars by the property that

$$
\bar{h}^{-t} \varphi_{\mathbb{C}}(\mathscr{C}) h^{-1}=\varphi_{\mathbb{C}}(\mathscr{C})
$$

for all $h \in H_{n}$. If $g \in \mathrm{SL}_{n}(\mathbb{C})$, the Heisenberg groups $H_{n}$ and $H_{n}^{\prime}$ of $\mathscr{C}$ and $g \cdot \mathscr{C}$ are related by $H_{n}^{\prime}=g H_{n} g^{-1}$. Then $g \cdot \varphi_{\mathbb{C}}(\mathscr{C})=\bar{g}^{-t} \varphi_{\mathbb{C}}(\mathscr{C}) g^{-1}$ is an $H_{n}^{\prime}$-invariant inner product, and so must be equal to $\varphi_{\mathbb{C}}(g \cdot \mathscr{C})$. Hence $\varphi_{\mathbb{C}}$ is $\mathrm{SL}_{n}(\mathbb{C})$-covariant. Moreover, since $H_{n} \subset \mathrm{SL}_{n}(\mathbb{C})$, this choice of covariant is forced on us. The compatibility with complex conjugation is seen in the same way.

Remark 6.2. In general $\varphi_{\mathbb{R}}$ is not the only $\mathrm{SL}_{n}(\mathbb{R})$-covariant. However, it is if the points of $E[n]$ are defined over $\mathbb{R}$, as happens in the case $n=2$ and $\Delta>0$ [Stoll and Cremona 2003, Lemma 3.2].

In practical terms, we have the following corollary.

Corollary 6.3. Let $M_{T} \in \mathrm{GL}_{n}(\mathbb{C})$ describe the action of $T \in E[n](\mathbb{C})$ on $\mathscr{b} \rightarrow \mathbb{P}^{n-1}$. Then the reduction covariant $\varphi_{\mathbb{C}}(\mathscr{C})$ is

$$
\sum_{T \in E[n](\mathbb{C})} \frac{1}{\left|\operatorname{det} M_{T}\right|^{2 / n}} \bar{M}_{T}^{t} M_{T} .
$$

Proof. To get an invariant inner product, we can take any inner product and average over its orbit under the action of $H_{n}$. Applying this to the standard inner product, we find that we can take, up to scaling,

$$
\varphi_{\mathbb{C}}(\mathscr{C})=\sum_{h \in H_{n}} \bar{h}^{-t} h^{-1}=\sum_{h \in H_{n}} \bar{h}^{t} h .
$$

In the statement of the corollary, $M_{T} \in \mathrm{GL}_{n}(\mathbb{C})$ is any lift of the element $\tau_{T}$ in $\mathrm{PGL}_{n}(\mathbb{C})$ describing the action of $T$ on $\mathbb{P}^{n-1}(\mathbb{C})$. The various preimages of $\tau_{T}$ in 
$H_{n}$ are given by $h=\alpha^{-1} M_{T}$ where $\alpha \in \mathbb{C}$ with $\alpha^{n}=\operatorname{det} M_{T}$. We then have

$$
\bar{h}^{t} h=\bar{\alpha}^{-1} \alpha^{-1} \bar{M}_{T}^{t} M_{T}=\frac{1}{\left|\operatorname{det} M_{T}\right|^{2 / n}} \bar{M}_{T}^{t} M_{T} .
$$

Since this only depends on $T$, it is sufficient to take the sum in (6-1) just over $T \in E[n](\mathbb{C})$, instead of over $h \in H_{n}$.

We can now define what we mean by a reduced genus-one normal curve.

Definition 6.4. A genus-one normal curve $\mathscr{C} \rightarrow \mathbb{P}^{n-1}$ defined over $\mathbb{R}$ is Minkowski reduced if $\varphi_{\mathbb{R}}(\mathscr{C})$ is the Gram matrix of a Minkowski reduced lattice basis, and likewise with "LLL" instead of "Minkowski".

Note that a lattice basis is (Minkowski or LLL) reduced if it is close to the standard basis of the standard lattice in the sense that the basis vectors are (short and) nearly orthogonal. The notion of a Minkowski reduced model has nice theoretical properties (it is optimal and essentially unique), whereas for practical purposes, it is important to be able to compute a reduced lattice basis efficiently; this is possible when using LLL reduced models.

If we start with some given (minimal) model $\mathscr{b} \rightarrow \mathbb{P}^{n-1}$, then in order to reduce it, we first compute its reduction covariant $\varphi_{\mathbb{R}}(\mathscr{C})$. We apply the LLL algorithm [Lenstra et al. 1982] to this Gram matrix, resulting in a unimodular transformation $U$ and an LLL reduced Gram matrix $M$, such that $M=U^{t} \varphi_{\mathbb{R}}(\mathscr{C}) U=U^{-1} \cdot \varphi_{\mathbb{R}}(\mathscr{C})$. We then apply the transformation $U^{-1}$ to our model $\mathscr{C}$. Since $\varphi_{\mathbb{R}}(\mathscr{C})$ is a covariant, we will have that $\varphi_{\mathbb{R}}\left(U^{-1} \cdot \mathscr{C}\right)=M$ is LLL reduced. Therefore $U^{-1} \cdot \mathscr{C}$ is the (minimal and) reduced model we are looking for.

Remark 6.5. Let $H: X_{n} \rightarrow X_{n}$ be the Hessian, as defined in [Fisher 2006a]. Then every nonsingular model in the pencil spanned by $\Phi$ and $H(\Phi)$ defines a curve with the same reduction covariant - since they have the same Heisenberg group. The case $n=2$ was previously given as [Cremona 1999, Propositions 10 and 13].

In the following subsections we discuss how to compute $\varphi_{\mathbb{R}}$. There are two basic approaches. One is to find the hyperosculating points of $\mathscr{C}(\mathbb{C})$ numerically and to compute the covariant from them. If $n=2$, we are looking for the ramification points of the covering $\mathscr{C} \rightarrow \mathbb{P}^{1}$; if $n=3$, for the flex points of the plane cubic curve $\mathscr{b} \subset \mathbb{P}^{2}$. The other approach is to use the $n$-torsion points in $E(\mathbb{C})$ instead and compute their action on $\mathbb{P}^{n-1}$. Generally speaking, the first approach leads to simpler formulas, whereas the second approach tends to be numerically more stable.

6B. Reduction of 2-coverings. We identify $\mathscr{H}_{2}^{+}(\mathbb{R})$ with the space of real positive definite binary quadratic forms, and $\mathscr{H}_{2}^{+}(\mathbb{R}) / \mathbb{R}_{>0}^{\times}$with the upper half-plane. This identification maps a real positive definite binary quadratic form to its unique root in the upper half-plane. 
Using the ramification points. Let $F(x, z) \in \mathbb{R}[x, z]$ be homogeneous of degree 4 . We assume that $f(X)=F(X, 1)$ has degree 4 as well. (If the leading coefficient is zero, make a change of coordinates first.) Let $\theta_{1}, \ldots, \theta_{4} \in \mathbb{C}$ be the roots of $f$. It is shown in [Stoll and Cremona 2003] that $\varphi_{\mathbb{R}}$ is given by

$$
\varphi_{\mathbb{R}}(F)(x, z)=\sum_{i=1}^{4} \frac{1}{\left|f^{\prime}\left(\theta_{i}\right)\right|}\left(x-\theta_{i} z\right)\left(x-\overline{\theta_{i}} z\right) .
$$

This goes back to [Julia 1917], where three different formulas are given according to the number of real roots of $f$; see also [Cremona 1999].

The formula is still valid for $\varphi_{\mathbb{C}}$, in the form

$$
\varphi_{\mathbb{C}}(F)(x, z)=\sum_{i=1}^{4} \frac{1}{\left|f^{\prime}\left(\theta_{i}\right)\right|}\left|x-\theta_{i} z\right|^{2} .
$$

In practice one should first numerically compute the roots of the resolvent cubic (which is not changed by reduction) and then compute the roots of $f$ from these.

Using the 2-torsion of $E$. The binary quartic

$$
F(x, z)=a x^{4}+b x^{3} z+c x^{2} z^{2}+d x z^{3}+e z^{4}
$$

has invariants $I$ and $J$ (see Section 2) and resolvent cubic $r(X)=X^{3}-3 I X+J$. For $\varphi$ a root of $r$ we set

$$
\begin{aligned}
& \alpha_{1}(\varphi)=4 a \varphi-8 a c+3 b^{2} \\
& \alpha_{2}(\varphi)=b \varphi-6 a d+b c \\
& \alpha_{3}(\varphi)=\frac{-2 \varphi^{2}+2 c \varphi-9 b d+4 c^{2}}{3},
\end{aligned}
$$

and

$$
W=\left(\begin{array}{cc}
0 & -1 \\
1 & 0
\end{array}\right), \quad A_{\varphi}=\left(\begin{array}{ll}
\alpha_{1}(\varphi) & \alpha_{2}(\varphi) \\
\alpha_{2}(\varphi) & \alpha_{3}(\varphi)
\end{array}\right) .
$$

Lemma 6.6. If $\alpha_{1}(\varphi) \neq 0$, then the action of the corresponding point $T \in E[2]$ on $\mathbb{P}^{1}$ is given by $M_{T}=W A_{\varphi}$.

Proof. Let $H(x, z)$ be the Hessian of $F$. The pencil spanned by $F$ and $H$ contains three degenerate quartics: for each root $\varphi^{\prime}$ of the resolvent cubic, we have

$$
\alpha_{1}\left(\varphi^{\prime}\right)\left(4 \varphi^{\prime} F(x, z)-\frac{1}{3} H(x, z)\right)=\left(\alpha_{1}\left(\varphi^{\prime}\right) x^{2}+2 \alpha_{2}\left(\varphi^{\prime}\right) x z+\alpha_{3}\left(\varphi^{\prime}\right) z^{2}\right)^{2} .
$$

Since the action of $T$ leaves both $F$ and $H$ invariant, $M_{T}$ must induce an involution on $\mathbb{P}^{1}$ that either fixes or swaps the roots of the quadratic on the right hand side; there is exactly one root $\varphi^{\prime}$ such that the roots of the corresponding quadratic are fixed. Therefore $\varphi^{\prime}=\varphi$, and the lemma follows by checking that $W A_{\varphi}$ does indeed fix the roots of the relevant quadratic. 
Lemma 6.7. If $M_{T} \in \mathrm{GL}_{2}$ describes the action of $T \in E[2]$ on $\mathscr{b} \rightarrow \mathbb{P}^{1}$ then

$$
\sum_{T \in E[2]} \frac{1}{\operatorname{det} M_{T}} M_{T}^{t} M_{T}=0 .
$$

Proof. We can verify this generically using the formula of Lemma 6.6.

Proposition 6.8. Let $F \in \mathbb{R}[x, z]$ be a nonsingular binary quartic, with resolvent cubic $r(X)=X^{3}-3 I X+J$.

(i) If $\Delta(F)>0$ then the reduction covariant is $\pm A_{\varphi}$ where $\varphi$ is the unique root of $r$ with $\operatorname{det}\left(A_{\varphi}\right)>0$ and the sign is that of $\alpha_{1}(\varphi)$.

(ii) If $\Delta(F)<0$ then the reduction covariant is

$$
\operatorname{Re}\left(\frac{1}{\left|\operatorname{det} A_{\varphi}\right|} \bar{A}_{\varphi} A_{\varphi}-\frac{1}{\operatorname{det} A_{\varphi}} A_{\varphi}^{2}\right)
$$

where $\varphi$ is a complex root of $r$.

Proof. If $\Delta(F)>0$, then $r$ has three real roots. Since $\operatorname{det}\left(A_{\varphi}\right)=-\alpha_{1}(\varphi) r^{\prime}(\varphi) / 3$, the analysis in [Cremona 1999] shows that there is a unique root $\varphi$ of $r$ with $\operatorname{det}\left(A_{\varphi}\right)>0$ (in particular, $\alpha_{1}(\varphi) \neq 0$ ). By Lemmas 6.6 and 6.7 the reduction covariant simplifies (up to a factor of 2) to

$$
\sum_{T \in E[2], \operatorname{det} M_{T}>0} \frac{1}{\operatorname{det} M_{T}} M_{T}^{t} M_{T}=I_{2}+\frac{1}{\operatorname{det} A_{\varphi}} A_{\varphi}^{2}=\frac{\operatorname{tr} A_{\varphi}}{\operatorname{det} A_{\varphi}} A_{\varphi},
$$

by the Cayley-Hamilton theorem. So $\pm A_{\varphi}$ is the positive definite symmetric matrix we are looking for, with the sign that makes the top left entry positive.

If $\Delta(F)<0$, then $r$ has a pair of complex conjugate roots, say $\varphi$ and $\bar{\varphi}$. If $E[2]=\{0, S, T, \bar{T}\}$, then we can take $M_{S}=M_{T} \bar{M}_{T}$, so

$$
\operatorname{det}\left(M_{S}\right)=\left|\operatorname{det}\left(M_{T}\right)\right|^{2}>0 .
$$

By Lemmas 6.6 and 6.7 again, the reduction covariant simplifies to

$$
\operatorname{Re}\left(\frac{1}{\left|\operatorname{det} M_{T}\right|} \bar{M}_{T}^{t} M_{T}-\frac{1}{\operatorname{det} M_{T}} M_{T}^{t} M_{T}\right)=\operatorname{Re}\left(\frac{1}{\left|\operatorname{det} A_{\varphi}\right|} \bar{A}_{\varphi} A_{\varphi}-\frac{1}{\operatorname{det} A_{\varphi}} A_{\varphi}^{2}\right) .
$$

Notice that we cannot have $\alpha_{1}(\varphi)=\alpha_{1}(\bar{\varphi})=0$, since then the resolvent cubic would have a repeated root, contradicting the fact that $F$ is nonsingular.

The cross terms. So far, we have shown how to find a unimodular transformation of the coordinates on $\mathbb{P}^{1}$ that reduces the 2 -covering. (If we start with a generalised binary quartic $(P, Q)$ then we work with $F=P^{2}+4 Q$.) There is still an ambiguity coming from the possibility of making a $y$-substitution in the general form of a 2covering. The most reasonable convention seems to be to arrange that the cross term coefficients $l, m$ and $n$ are 0 or 1 . 


\section{C. Reduction of 3-coverings.}

Using the flex points. Let $F(x, y, z) \in \mathbb{R}[x, y, z]$ be a nonsingular ternary cubic. In order to find its reduction covariant (as a positive definite quadratic form $Q(x, y, z))$, we proceed as follows. Let $H(x, y, z)$ be the Hessian of $F$ as defined in Section 2. Then the intersection of $F=0$ and $H=0$ consists of nine distinct points, the flex points of $F$. Three of them are real, the others come in three complex conjugate pairs.

There are twelve lines each containing three of the flex points, coming in four triples of lines that do not meet in a flex point. (These triples are the "syzygetic triangles" mentioned below.) One of these triples has all three lines real, call them $L_{11}, L_{12}$ and $L_{13}$. Another one has one line real, call it $L_{21}$, and two complex conjugate lines, call them $L_{22}$ and $L_{23}$. Then $Q$ spans the one-dimensional intersection of the spaces spanned by $L_{11}^{2}, L_{12}^{2}$ and $L_{13}^{2}$, and by $L_{21}^{2}$ and $L_{22} L_{23}$, respectively.

In order to see why this recipe works, first observe that it clearly defines an $\mathrm{SL}_{3}(\mathbb{R})$-covariant map. We can always make an $\mathrm{SL}_{3}(\mathbb{R})$-transformation to bring $F$ into the standard Hesse form

$$
F(x, y, z)=a\left(x^{3}+y^{3}+z^{3}\right)+b x y z .
$$

Then $L_{11}, L_{22}$ and $L_{33}$ are $x, y$ and $z$, and $L_{21}, L_{22}$ and $L_{23}$ are $x+y+z, x+$ $\zeta_{3} y+\zeta_{3}^{2} z$ and $x+\zeta_{3}^{2} y+\zeta_{3} z$ (where $\zeta_{3}$ is a primitive cube root of unity). One then looks at the intersection

$$
\left\langle x^{2}, y^{2}, z^{2}\right\rangle \cap\left\langle(x+y+z)^{2}, x^{2}+y^{2}+z^{2}-x y-y z-z x\right\rangle
$$

and finds it is one-dimensional, spanned by $x^{2}+y^{2}+z^{2}$, which is the reduction covariant of any $F$ in Hesse form.

The only way we know to implement this method in practice is by numerically solving for the flex points. If the given model is far from reduced, then usually several of the flex points are very close to one another, which makes the computation of the lines difficult. Another practical problem is that the two spaces of quadrics we compute are only approximate and therefore will usually not have a nontrivial intersection.

Using the 3-torsion on E. This is the method described in [Fisher 2006b, §9.5]. Let $F(x, y, z)$ be a ternary cubic with invariants $c_{4}$ and $c_{6}$ and Hessian $H$ as defined in Section 2. Let $T=\left(x_{T}, y_{T}\right)$ be a 3-torsion point on the Jacobian

$$
E: \quad y^{2}=x^{3}-27 c_{4} x-54 c_{6} .
$$

Then the cubic $\mathscr{T}(x, y, z)=2 x_{T} F-3 H$ is the product of 3 linear forms. (In [Hilbert 1993, II.7] it is called a "syzygetic triangle".) Making a change of coordinates (if 
necessary) we may suppose $\mathscr{T}(1,0,0) \neq 0$. We label the coefficients $\mathscr{T}(x, y, z)=r x^{3}+s_{1} x^{2} y+s_{2} x y^{2}+s_{3} y^{3}+t_{1} x^{2} z+t_{2} x z^{2}+t_{3} z^{3}+u x y z+v y^{2} z+w y z^{2}$.

The proof of [Fisher 2006b, Theorem 7.1] describes how to compute a formula for $M_{T}$, where the entries are polynomials in $r, s_{1}, s_{2}, \ldots, w$ and $y_{T}$. Up to a scaling, this works out as $M_{T}=r A+2 y_{T} B$ where

$$
\begin{aligned}
& A_{11}=-12 r s_{2} w-36 r s_{3} t_{2}+12 r u v+4 s_{1}^{2} w+4 s_{1} s_{2} t_{2}-8 s_{1} t_{1} v-s_{1} u^{2}+12 s_{3} t_{1}^{2}, \\
& A_{12}=-54 r s_{3} w+18 r v^{2}+6 s_{1} s_{2} w-3 s_{1} u v-6 s_{2} t_{1} v+9 s_{3} t_{1} u, \\
& A_{13}=-81 r s_{3} t_{3}+9 r v w+9 s_{1} s_{2} t_{3}-3 s_{1} t_{2} v-3 s_{2} t_{1} w+9 s_{3} t_{1} t_{2}, \\
& A_{21}=36 r s_{2} t_{2}-9 r u^{2}-12 s_{1}^{2} t_{2}+12 s_{1} t_{1} u-12 s_{2} t_{1}^{2}, \\
& A_{22}=24 r s_{2} w+18 r s_{3} t_{2}-15 r u v-8 s_{1}^{2} w-2 s_{1} s_{2} t_{2}+10 s_{1} t_{1} v+2 s_{1} u^{2}-3 s_{2} t_{1} u-6 s_{3} t_{1}^{2}, \\
& A_{23}=54 r s_{2} t_{3}-9 r u w-18 s_{1}^{2} t_{3}+6 s_{1} t_{1} w+3 s_{1} t_{2} u-6 s_{2} t_{1} t_{2}, \\
& A_{31}=0,
\end{aligned}
$$$$
A_{32}=-18 r s_{2} v+27 r s_{3} u+6 s_{1}^{2} v-3 s_{1} s_{2} u-18 s_{1} s_{3} t_{1}+6 s_{2}^{2} t_{1},
$$$$
A_{33}=-12 r s_{2} w+18 r s_{3} t_{2}+3 r u v+4 s_{1}^{2} w-2 s_{1} s_{2} t_{2}-2 s_{1} t_{1} v-s_{1} u^{2}+3 s_{2} t_{1} u-6 s_{3} t_{1}^{2}
$$$$
\text { and } B=r B_{1}+\left(s_{1}^{2} t_{2}-s_{1} t_{1} u+s_{2} t_{1}^{2}\right) E_{13} \text { with }
$$

$$
B_{1}=\left(\begin{array}{ccc}
s_{1} u-2 s_{2} t_{1} & s_{1} v-3 s_{3} t_{1} & s_{1} w-4 s_{2} t_{2}-t_{1} v+u^{2} \\
-3 r u+2 s_{1} t_{1} & -3 r v+s_{2} t_{1} & -3 r w+s_{1} t_{2} \\
6 r s_{2}-2 s_{1}^{2} & 9 r s_{3}-s_{1} s_{2} & 3 r v-s_{1} u+s_{2} t_{1}
\end{array}\right) .
$$

(Notes: $E_{i j}$ is the 3 by 3 matrix with $(i, j)$ entry 1 and all other entries 0 . Our matrices $A$ and $B$ would be called $r^{3}(\operatorname{det} P) A$ and $r^{3} B$ in the notation of [Fisher 2006b].) This formula comes with the caveat (see [Fisher 2006b, Remark 7.2]) that it may give zero. However, as this will not happen for both $T$ and $-T$, we may get around the problem by computing $M_{T}$ as $\left(M_{-T}\right)^{-1}$ if necessary.

Once we have computed $M_{T}$ for all $T \in E[3]$ the reduction covariant is computed using Corollary 6.3.

6D. Reduction of 4-coverings. We could again try to find the reduction covariant starting from the 16 hyperosculating points on $\mathscr{b}$ and the quadruples of planes containing four of them, which are the analogue of the syzygetic triangles. However, this approach does not seem to be very promising.

Instead, we use the fact that below the given 4 -covering $\mathscr{C}$, there is a 2 -covering $\mathscr{C}_{2}$; let $\pi: \mathscr{C} \rightarrow \mathscr{C}_{2}$ be the covering map. If $A$ and $B$ are the symmetric matrices corresponding to the quadrics defining $\mathscr{C} \subset \mathbb{P}^{3}$, then $\mathscr{C}_{2}$ has equation $y^{2}=F(x, z)$ where

$$
F(x, z):=\operatorname{det}(A x+B z)
$$


Applying reduction to the quartic on the right hand side, we find a good basis of the pencil of quadrics. It remains to find the reduction covariant of $\mathscr{b}$.

Let $\theta_{j} \in \mathbb{C}(j=1,2,3,4)$ be the ramification points of $\mathscr{C}_{2} \rightarrow \mathbb{P}^{1}$, i.e., the roots of $f(X)=F(X, 1)$. Let $G_{j}(j=1,2,3,4)$ be a linear form (unique up to scaling) describing the preimage of $\theta_{j}$ on $\mathscr{C} \subset \mathbb{P}^{3}$. Then (fixing the polynomials giving the covering map $\left.\pi: \mathscr{C} \rightarrow \mathscr{C}_{2}\right)$ there are $\alpha_{j} \in \mathbb{C}^{\times}$such that

$$
\left(x-\theta_{j} z\right) \circ \pi=\alpha_{j} G_{j}^{2} .
$$

Now the action of $T \in E[4]$ on $\mathscr{C}$ induces the action of $2 T \in E[2]$ on $\mathscr{C}_{2}$. Therefore

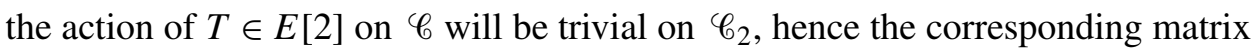
$M_{T} \in \mathrm{SL}_{4}$ will fix the $G_{j}$ up to sign. In fact, it can be checked that the action of $E$ [2] on $\mathbb{P}^{3}$ lifts to a representation on $\mathbb{C}^{4}$, which is isomorphic to the regular representation, and the $G_{j}$ span the four eigenspaces. So any Hermitian form that is invariant under $H_{4}$ must be invariant under $E$ [2] and thus be of the form

$$
\sum_{j=1}^{4} \lambda_{j}\left|G_{j}\right|^{2}
$$

It remains to determine the coefficients $\lambda_{j}$.

Proposition 6.9. Keep the notation introduced so far, and let $f(X)=F(X, 1)$. Then the reduction covariant of $\mathscr{C}$ is the positive definite Hermitian form

$$
\varphi_{\mathbb{C}}(\mathscr{b})=\sum_{j=1}^{4} \frac{\left|\alpha_{j}\right|}{\left|f^{\prime}\left(\theta_{j}\right)\right|^{1 / 2}}\left|G_{j}\right|^{2} .
$$

If $\mathscr{C}$ is defined over $\mathbb{R}$, then the restriction of this Hermitian form to $\mathbb{R}^{4}$ will be the positive definite quadratic form $\varphi_{\mathbb{R}}(\mathscr{C})$.

Proof. We first check that the given form is invariant under $\mathrm{SL}_{2}(\mathbb{C})$ acting on $\mathbb{P}^{1}$ (that is, does not depend on the choice of basis of the pencil of quadrics). We know (see page 805) that $\sum_{j=1}^{4}\left|f^{\prime}\left(\theta_{j}\right)\right|^{-1}\left|x-\theta_{j} z\right|^{2}$ is an $\mathrm{SL}_{2}(\mathbb{C})$-covariant; the same computation (which deals with each summand separately) shows that $\sum_{j=1}^{4}\left|f^{\prime}\left(\theta_{j}\right)\right|^{-1 / 2}\left|x-\theta_{j} z\right|$ is a covariant as well. But $\left|x-\theta_{j} z\right|=\left|\alpha_{j} G_{j}^{2}\right|$, and the coordinates in $G_{j}$ are not affected by the $\mathrm{SL}_{2}(\mathbb{C})$-action, so the expression given in the statement is invariant.

Now we check that the given form is covariant with respect to the action of $\mathrm{SL}_{4}(\mathbb{C})$. But this is clear since every $\alpha_{j} G_{j}^{2}$ is covariant.

Since we can move any $\mathscr{C}$ into standard form by the action of $\mathrm{SL}_{2}(\mathbb{C}) \times \mathrm{SL}_{4}(\mathbb{C})$, it now suffices to verify that our formula gives the correct result when $\mathscr{C}$ is in standard form

$$
a\left(x_{0}^{2}+x_{2}^{2}\right)+2 b x_{1} x_{3}=a\left(x_{1}^{2}+x_{3}^{2}\right)+2 b x_{0} x_{2}=0 .
$$


In this case, the 2 -covering $\mathscr{C}_{2}$ is given by

$$
y^{2}=\left(a^{4}+b^{4}\right) x^{2} z^{2}-a^{2} b^{2}\left(x^{4}+z^{4}\right)
$$

and the map $\pi$ (see Lemma 4.6 for formulae), followed by the map $\mathscr{C}_{2} \rightarrow \mathbb{P}^{1}$, is given by

$$
(x: z)=\left(b^{3}\left(x_{1}^{2}+x_{3}^{2}\right)+2 a^{3} x_{0} x_{2}:-b^{3}\left(x_{0}^{2}+x_{2}^{2}\right)-2 a^{3} x_{1} x_{3}\right) .
$$

The roots $\theta_{j}$ of $f(X)=-a^{2} b^{2} X^{4}+\left(a^{4}+b^{4}\right) X^{2}-a^{2} b^{2}$ are $a / b,-a / b, b / a$ and $-b / a$, and up to a common factor $b^{4}-a^{4}$, we can take $\alpha_{j}=1 / b, 1 / b, 1 / a,-1 / a$ and $G_{j}=x_{1}-x_{3}, x_{1}+x_{3}, x_{0}-x_{2}, x_{0}+x_{2}$. Also, $\left|f^{\prime}\left(\theta_{j}\right)\right|=c\left|\theta_{j}\right|$ for some constant $c$. Since $\left|\alpha_{j}\right| /\left|\theta_{j}\right|^{1 / 2}$ has the same value $|a b|^{-1 / 2}$ for all $j$, our expression gives, up to a constant factor again,

$$
\left|x_{1}-x_{3}\right|^{2}+\left|x_{1}+x_{3}\right|^{2}+\left|x_{0}-x_{2}\right|^{2}+\left|x_{0}+x_{2}\right|^{2}=2\left(\left|x_{0}\right|^{2}+\left|x_{1}\right|^{2}+\left|x_{2}\right|^{2}+\left|x_{3}\right|\right),
$$

which is the correct result for a 4-covering in standard form.

In order to find the $\alpha_{j}$ and $G_{j}$, we can make use of a result from [Fisher 2008b], where it is observed that $\alpha_{j} G_{j}^{2}$ is the quadratic form corresponding to the matrix

$$
e \theta_{j}^{-1} A+M_{1}+\theta_{j} M_{2}+a \theta_{j}^{2} B
$$

here $F(x, z)=\operatorname{det}(A x+B z)=a x^{4}+b x^{3} z+c x^{2} z^{2}+d x z^{3}+e z^{4}$ and $M_{1}$ and $M_{2}$ are obtained from relation (4-3).

\section{Examples}

In this section we illustrate minimisation and reduction for two explicit examples over $\mathbb{Q}$ (one a 3-covering and the other a 4-covering). We then give references to further examples.

7A. Minimisation and reduction of a 3-covering. We consider the elliptic curve $105630 \mathrm{~d} 1$ in [Cremona n.d.] with Weierstrass equation

$$
E: \quad y^{2}+x y=x^{3}+x^{2}-114848533 x-472424007827 .
$$

Computing the 3-Selmer group [Schaefer and Stoll 2004] we find $\operatorname{Sel}^{(3)}(\mathbb{Q}, E) \cong$ $\mathbb{Z} / 3 \mathbb{Z}$. In [Cremona et al. 2008; 2009; n.d.] we show how to write down elements of the 3-Selmer group explicitly as 3-coverings of $E$. In this case our MAGMA programs find (before minimisation and reduction) that a generator is represented by the 3 -covering $\mathscr{C} \subset \mathbb{P}^{2}$ with equation

$$
\begin{aligned}
F_{1}(x, y, z)=27089 x^{3}+2142 y^{3}+291938 z^{3}+10008 x^{2} y-127341 x^{2} z \\
+92937 x y^{2}+104736 y^{2} z+21093 x z^{2}-71172 y z^{2}-2655 x y z .
\end{aligned}
$$


(Random choices in the programs mean it need not return the same cubic every time. However, the answer will always be $\mathbb{Q}$-equivalent to $F_{1}$, and this can be checked using the algorithm in [Fisher 2006b].) The discriminant of this ternary cubic is $\Delta\left(F_{1}\right)=3^{12} \cdot 503^{12} \cdot \Delta_{E}$ where $\Delta_{E}=2^{39} \cdot 3 \cdot 5^{9} \cdot 7^{3} \cdot 503$ is the minimal discriminant of $E$. So $F_{1}$ has level 1 at the primes 3 and 503. Reducing mod 3 we find $F_{1}(x, y, z)=2(x+z)^{3}(\bmod 3)$. The level is decreased by the first iteration of our algorithm (see Theorem 4.3). Explicitly, we put

$$
F_{2}(x, y, z)=\frac{1}{3^{2}} F_{1}(3 x-y, z, y) .
$$

Likewise we find $F_{2}(x, y, z) \equiv 284(x+329 y+33 z)^{3}(\bmod 503)$ and our algorithm puts

$$
\begin{aligned}
F_{3}(x, y, z)= & \frac{1}{503^{2}} F_{2}(503 x-33 y+z, z, y-10 z) \\
= & 40877301 x^{3}-11504 y^{3}+12 z^{3}-8035425 x^{2} y-64887 x^{2} z \\
& +526580 x y^{2}-200 y^{2} z+5803 x z^{2}-383 y z^{2}+7307 x y z .
\end{aligned}
$$

The 3-torsion of $y^{2}=x^{3}-27 c_{4} x-54 c_{6}$ over $\mathbb{C}$ is generated by

$$
\begin{aligned}
& S=(667989.968057,420236746.168), \\
& T=(-264330.994609,34120617.5970 i) .
\end{aligned}
$$

The formulae in Section 6C show that $S$ and $T$ act on $\left\{F_{3}=0\right\}$ via

$$
M_{S}=\left(\begin{array}{lll}
285.46 & -19.022 & 3.4264 \\
4352.6 & -290.04 & 52.341 \\
509.05 & -33.785 & 4.5806
\end{array}\right)
$$

(entries being given to five-digit precision) and

$$
M_{T}=\left(\begin{array}{rrr}
-50.656+47.060 i & 3.2758-3.3464 i & 0.11909+2.2683 i \\
-786.55+717.15 i & 50.871-51.000 i & 1.8675+34.587 i \\
-119.84+93.073 i & 7.8268-6.5354 i & -0.21547+3.9405 i
\end{array}\right)
$$

We have scaled these matrices to have determinant 1 . By Corollary 6.3 the matrix of the reduction covariant (given to twelve-digit precision) is

$$
A=\left(\begin{array}{rrr}
176413988.185 & -11560848.1174 & 3471.84429193 \\
-11560848.1174 & 757736.524016 & -1499.92503970 \\
3471.84429193 & -1499.92503970 & 13237.5156939
\end{array}\right) \text {. }
$$


Running the LLL algorithm on the lattice with Gram matrix $A$ results in the unimodular transformation

$$
U=\left(\begin{array}{rrr}
0 & 0 & 1 \\
4 & 61 & 6 \\
-3 & -46 & -4
\end{array}\right)
$$

Accordingly we put $F_{4}(x, y, z)=F_{3}(4 y-3 z, 61 y-46 z, x+6 y-4 z)$ and find

$$
\begin{aligned}
& F_{4}(x, y, z)= \\
& \qquad 12 x^{3}+12 y^{3}+171 z^{3}+65 x^{2} y+65 x^{2} z-94 y^{2} z+87 x z^{2}+101 y z^{2}+7 x y z .
\end{aligned}
$$

This ternary cubic has solution

$$
(x: y: z)=(345420:-1638959:-373029),
$$

which by the formulae in [An et al. 2001] maps down to a point

$$
\begin{aligned}
& x=\frac{-74872620773608422623058757914981065217}{109435039457696221^{2}}, \\
& y=\frac{51043047025320389176098494307847798722958228061916407587}{109435039457696221^{3}}
\end{aligned}
$$

on $E(\mathbb{Q})$ of canonical height $86.5313 \ldots$ Since the torsion subgroup of $E(\mathbb{Q})$ is trivial, it follows that $\operatorname{rank} E(\mathbb{Q})=1$. It is equally convenient to find this generator using Heegner points.

Note that the MAGMA implementation of 3-descent does the minimisation and reduction automatically. To extract the intermediate model $F_{1}(x, y, z)=0$, one should first specify that 3-descent prints out some of its working, using the command SetVerbose ("ThreeDescent", 1);

7B. Minimisation and reduction of a 4-covering. In [Skorobogatov 2001, §8.1], an example is given of a 4-covering $\mathscr{C}$ of the elliptic curve $E: y^{2}=x^{3}-1221$ that represents an element of exact order 4 in the Shafarevich-Tate group of $E$. The symmetric matrices corresponding to the two quadrics defining $\mathscr{C} \subset \mathbb{P}^{3}$ are given as (to keep with our convention, we multiply by 2 so that entries are the second partial derivatives)

$$
A=2\left(\begin{array}{rrrr}
-1 & 11 & -66 & 396 \\
11 & -66 & 396 & -2520 \\
-66 & 396 & -2520 & 16335 \\
396 & -2520 & 16335 & -105786
\end{array}\right), \quad B=2\left(\begin{array}{rrrr}
-1 & -3 & 33 & -198 \\
-3 & 33 & -198 & 1188 \\
33 & -198 & 1188 & -7560 \\
-198 & 1188 & -7560 & 49005
\end{array}\right) .
$$

We will use $x_{1}, \ldots, x_{4}$ as the coordinates on $\mathbb{P}^{3}$. We find that

$$
\operatorname{det}(A x+B z)=2^{4} \cdot 3^{8}\left(-9 x^{4}+13 x^{3} z-18 x^{2} z^{2}+3 z^{4}\right),
$$


which makes it clear that the model is non-minimal at $p=2$ and $p=3$. We compute that the discriminant of our quadric intersection is $\left(2 \cdot 3^{4}\right)^{12}$ times the (minimal) discriminant $-2^{4} 3^{5} 11^{2} 37^{2}$ of $E$, which shows that the level at 2 is 1 and the level at 3 is 4 ; the model is already minimal at all other primes.

We first minimise at $p=3$. According to our algorithm (see Section 4C), we have to look at the reductions of $A$ and $B \bmod 3$, which are

$$
\bar{A}=\left(\begin{array}{llll}
1 & 1 & 0 & 0 \\
1 & 0 & 0 & 0 \\
0 & 0 & 0 & 0 \\
0 & 0 & 0 & 0
\end{array}\right) \quad \text { and } \quad \bar{B}=\left(\begin{array}{llll}
1 & 0 & 0 & 0 \\
0 & 0 & 0 & 0 \\
0 & 0 & 0 & 0 \\
0 & 0 & 0 & 0
\end{array}\right)
$$

The common nullity is $s=2$, and the reduced quadratic forms already involve only the first two variables. They represent zero simultaneously over $\mathbb{F}_{3}$; the plane $x_{1}=0$ is contained in the reduction of the curve. So we apply the transformation $\left[\frac{1}{3} I_{2}, \operatorname{Diag}(3,1,1,1)\right]$, resulting in the new pair of matrices (which we will again denote by $A$ and $B$ )

$A=\left(\begin{array}{rrrr}-6 & 22 & -132 & 792 \\ 22 & -44 & 264 & -1680 \\ -132 & 264 & -1680 & 10890 \\ 792 & -1680 & 10890 & -70524\end{array}\right), \quad B=\left(\begin{array}{rrrr}-6 & -6 & 66 & -396 \\ -6 & 22 & -132 & 792 \\ 66 & -132 & 792 & -5040 \\ -396 & 792 & -5040 & 32670\end{array}\right)$

The level at $p=3$ of the new model is 3 . Reducing mod 3, we have now

$$
\bar{A}=\left(\begin{array}{llll}
0 & 1 & 0 & 0 \\
1 & 1 & 0 & 0 \\
0 & 0 & 0 & 0 \\
0 & 0 & 0 & 0
\end{array}\right), \quad \bar{B}=\left(\begin{array}{llll}
0 & 0 & 0 & 0 \\
0 & 1 & 0 & 0 \\
0 & 0 & 0 & 0 \\
0 & 0 & 0 & 0
\end{array}\right)
$$

The common nullity is again $s=2$, and there is a plane contained in the reduction. This time, the plane is $x_{2}=0$, so we swap $x_{1}$ and $x_{2}$ before we apply $\left[\frac{1}{3} I_{2}, \operatorname{Diag}(3,1,1,1)\right]$. The result is a model of level 2 :

$$
A=\left(\begin{array}{rrrr}
-132 & 22 & 264 & -1680 \\
22 & -2 & -44 & 264 \\
264 & -44 & -560 & 3630 \\
-1680 & 264 & 3630 & -23508
\end{array}\right), \quad B=\left(\begin{array}{rrrr}
66 & -6 & -132 & 792 \\
-6 & -2 & 22 & -132 \\
-132 & 22 & 264 & -1680 \\
792 & -132 & -1680 & 10890
\end{array}\right)
$$

Now we get a different situation mod 3:

$$
\bar{A}=\left(\begin{array}{llll}
0 & 1 & 0 & 0 \\
1 & 1 & 1 & 0 \\
0 & 1 & 1 & 0 \\
0 & 0 & 0 & 0
\end{array}\right), \quad \bar{B}=\left(\begin{array}{llll}
0 & 0 & 0 & 0 \\
0 & 1 & 1 & 0 \\
0 & 1 & 0 & 0 \\
0 & 0 & 0 & 0
\end{array}\right)
$$


The common nullity is $s=1$. We swap $x_{1}$ and $x_{4}$ so that the reduced forms only involve the last three variables. Then we see that we are in 'Situation 2', so we apply the transformation $\left[I_{2}, \operatorname{Diag}\left(\frac{1}{3}, 1,1,1\right)\right]$. This results in a model of level 1 , given by

$$
A=\left(\begin{array}{rrrr}
-2612 & 88 & 1210 & -560 \\
88 & -2 & -44 & 22 \\
1210 & -44 & -560 & 264 \\
-560 & 22 & 264 & -132
\end{array}\right), \quad B=\left(\begin{array}{rrrr}
1210 & -44 & -560 & 264 \\
-44 & -2 & 22 & -6 \\
-560 & 22 & 264 & -132 \\
264 & -6 & -132 & 66
\end{array}\right)
$$

In the last minimisation step at $p=3$, the reductions are now

$$
\bar{A}=\left(\begin{array}{llll}
1 & 1 & 1 & 1 \\
1 & 1 & 1 & 1 \\
1 & 1 & 1 & 0 \\
1 & 1 & 0 & 0
\end{array}\right), \quad \bar{B}=\left(\begin{array}{llll}
1 & 1 & 1 & 0 \\
1 & 1 & 1 & 0 \\
1 & 1 & 0 & 0 \\
0 & 0 & 0 & 0
\end{array}\right)
$$

The common nullity is once more $s=1$, while the common kernel is spanned by $(1,-1,0,0)$. We move it to $(1,0,0,0)$ and are in Situation 2 again. After applying $\left[I_{2}, \operatorname{Diag}\left(\frac{1}{3}, 1,1,1\right)\right]$, we obtain a model that is now minimal at $p=3$.

$$
A=\left(\begin{array}{rrrr}
-310 & 30 & 418 & -194 \\
30 & -2 & -44 & 22 \\
418 & -44 & -560 & 264 \\
-194 & 22 & 264 & -132
\end{array}\right), \quad B=\left(\begin{array}{rrrr}
144 & -14 & -194 & 90 \\
-14 & -2 & 22 & -6 \\
-194 & 22 & 264 & -132 \\
90 & -6 & -132 & 66
\end{array}\right)
$$

We still have to minimise at $p=2$, using the algorithm described in Section 4D. We first find the "double" of our model:

$$
\begin{aligned}
\mathfrak{d}^{\prime}(A, B)=(P, Q)= & \left(2^{2}\left(6413 x^{2}-5665 x z+1248 z^{2}\right)\right. \\
& 2^{2}\left(41126578 x^{4}-72659303 x^{3} z\right. \\
& \left.\left.+48099091 x^{2} z^{2}-14139840 x z^{3}+1557501 z^{4}\right)\right)
\end{aligned}
$$

We see that we already have $v_{2}(P) \geq 1$ and $v_{2}(Q) \geq 2$. The common kernel of the reductions mod 2 of the two quadratic forms is spanned by $(1,1,0,1)$ and $(0,0,1,0)$, so the common nullity is $s=2$. We change coordinates so that the common kernel is given by $x_{1}=x_{2}=0$. Then the reductions of the quadrics are $x_{1}^{2}$ and $x_{2}^{2}$, so they do not simultaneously represent zero. We apply the 'flip-flop' transformation $\left[\frac{1}{2} I_{2}, \operatorname{Diag}(2,2,1,1)\right]$, after which the reductions are $x_{3} x_{4}$ and $x_{4}^{2}$, so now there is the plane $x_{4}=0$ contained in the reduction of the curve. We swap $x_{1}$ and $x_{4}$ and then apply $\left[\frac{1}{2} I_{2}, \operatorname{Diag}(2,1,1,1)\right]$ to obtain a pair of matrices 
representing a globally minimal model:

$$
A=\left(\begin{array}{rrrr}
-728 & -424 & 319 & -474 \\
-424 & -252 & 187 & -280 \\
319 & 187 & -140 & 209 \\
-474 & -280 & 209 & -310
\end{array}\right), \quad B=\left(\begin{array}{rrrr}
348 & 198 & -152 & 220 \\
198 & 114 & -86 & 130 \\
-152 & -86 & 66 & -97 \\
220 & 130 & -97 & 144
\end{array}\right)
$$

We now apply reduction to this model as described in Section 6D. We have

$$
\operatorname{det}(A x+B z)=4\left(-9 x^{4}+13 x^{3} z-18 x^{2} z^{2}+3 z^{4}\right) .
$$

Following [An et al. 2001] and [Fisher 2008b], we compute the quadratic forms $T_{1}, T_{2}$ whose symmetric matrices $M_{1}, M_{2}$ are given by

$$
\operatorname{adj}(\operatorname{adj}(A) x+\operatorname{adj}(B) z))=4^{2} \cdot 81 A x^{3}-4 \cdot 9 M_{1} x^{2} z+4 \cdot 3 M_{2} x z^{2}+4^{2} \cdot 9 B z^{3} .
$$

Then, writing $Q_{1}$ and $Q_{2}$ for the quadratic forms corresponding to $A$ and $B$,

$$
\alpha G^{2}=12 \theta^{-1} Q_{1}+T_{1}+\theta T_{2}-36 \theta^{2} Q_{2}
$$

for $\theta$ a root of $f(X)=\operatorname{det}(X A+B)$. We can for example take $G=\left(-18 \theta^{3}-28 \theta^{2}+6 \theta+2\right) x_{1}+\left(18 \theta^{3}-26 \theta^{2}+2\right) x_{2}+\left(18 \theta^{2}+\theta-3\right) x_{3}-2 x_{4}$ and $\alpha=-1395 \theta^{3}+1367 \theta^{2}-2155 \theta-1001$. Also, $f^{\prime}(\theta)=12\left(-12 \theta^{3}+13 \theta^{2}-12 \theta\right)$. The matrix corresponding to $\sqrt{12} \sum_{\theta}|\alpha||G|^{2} /\left|f^{\prime}(\theta)\right|^{1 / 2}$ is (to five decimal places)

$$
\left(\begin{array}{rrrr}
8857.72019 & 5117.00780 & -3885.97776 & 5665.67630 \\
5117.00780 & 3080.24124 & -2279.16858 & 3348.18401 \\
-3885.97776 & -2279.16858 & 1716.07038 & -2498.36286 \\
5665.67630 & 3348.18401 & -2498.36286 & 3706.96839
\end{array}\right) .
$$

We apply LLL to this Gram matrix and obtain the reducing transformation matrix

$$
U=\left(\begin{array}{rrrr}
-5 & -2 & -6 & 0 \\
-6 & -3 & -7 & -1 \\
-15 & -7 & -17 & 0 \\
3 & 1 & 4 & 1
\end{array}\right)
$$

which finally brings the two matrices defining $\mathscr{C}$ into the form

$$
U^{t} A U=\left(\begin{array}{rrrr}
-2 & 0 & -1 & -2 \\
0 & -2 & -1 & 0 \\
-1 & -1 & -2 & 2 \\
-2 & 0 & 2 & -2
\end{array}\right), \quad U^{t} B U=\left(\begin{array}{rrrr}
0 & 0 & -1 & 1 \\
0 & 2 & -1 & -1 \\
-1 & -1 & 0 & -1 \\
1 & -1 & -1 & -2
\end{array}\right) .
$$


These correspond, after a sign change, to the quadratic forms

$$
\begin{aligned}
& Q_{1}=x_{1}^{2}+x_{1} x_{3}+2 x_{1} x_{4}+x_{2}^{2}+x_{2} x_{3}+x_{3}^{2}-2 x_{3} x_{4}+x_{4}^{2}, \\
& Q_{2}=x_{1} x_{3}-x_{1} x_{4}-x_{2}^{2}+x_{2} x_{3}+x_{2} x_{4}+x_{3} x_{4}+x_{4}^{2} .
\end{aligned}
$$

7C. Further examples and applications. One useful application of the methods described in this paper is to help find large generators in the Mordell-Weil group of an elliptic curve $E$. This has already be demonstrated in Section 7A. Each rational point $P \in E(\mathbb{Q})$ lifts to one of the $n$-coverings of $E$. If we have a nice and small (i.e., minimised and reduced) model $\mathscr{C}$ of this $n$-covering, then the logarithmic height with respect to $\mathscr{C} \rightarrow \mathbb{P}^{n-1}$ of the preimage $Q$ of $P$ in $\mathscr{C}(\mathbb{Q})$ will be smaller by a factor of about $\frac{1}{2 n}$ than the logarithmic $x$-coordinate height of $P-$ standard properties of heights imply that

$$
h(Q)=\frac{1}{2 n} h_{x}(P)+O(1)
$$

where the implied constant depends on the equations defining $\mathscr{b} \rightarrow \mathbb{P}^{n-1}$. If the equations have small coefficients, this constant should be small as well. Therefore we can hope to find $P$ much more easily by searching for $Q$ on $\mathscr{C}$. In fact, this application was the motivation for the first tentative steps towards reduction of 4coverings. The story begins with [Gebel et al. 1998], where the authors determined Mordell-Weil generators for all Mordell curves $y^{2}=x^{3}+D$, with $D$ a nonzero integer of absolute value at most $10^{4}$ (in order to determine all the integral points on these curves), with one exception, $D=7823$. The analytic rank of this curve is 1 , so we know that the Mordell-Weil rank must be also 1; however the BirchSwinnerton-Dyer Conjecture predicts a generator of fairly large height. One of us (Stoll) used minimisation and reduction of 4-coverings in a fairly ad hoc fashion to find a good model of the one relevant 4-covering of $E: y^{2}=x^{3}+7823$, so that a point search on this 4-covering curve was successful, thus resolving this last open case. The result was reported in a posting [Stoll 2002] to the NMBRTHRY mailing list. We give a short summary of the steps and the result. By a standard 2-descent, one obtains a 2-covering curve

$$
C: y^{2}=-18 x^{4}+116 x^{3}+48 x^{2}-12 x+30 .
$$

A second 2-descent on $C$ following [Merriman et al. 1996] produces a 4-covering of $E$, whose initial model was given by quadrics with coefficients of up to $15 \mathrm{dec}-$ imal digits. Using the methods described here, one finds a model $D \subset \mathbb{P}^{3}$ given by

$$
\begin{array}{r}
2 x_{1} x_{2}+x_{1} x_{3}+x_{1} x_{4}+x_{2} x_{4}+x_{3}^{2}-2 x_{4}^{2}=0 \\
x_{1}^{2}+x_{1} x_{3}-x_{1} x_{4}+2 x_{2}^{2}-x_{2} x_{3}+2 x_{2} x_{4}-x_{3}^{2}-x_{3} x_{4}+x_{4}^{2}=0 .
\end{array}
$$


It is not very difficult to find the point $P=(116: 207: 474:-332)$ on $D$. This point then gives rise to the point

$$
Q=\left(\frac{53463613}{32109353}, \frac{23963346820191122}{32109353^{2}}\right)
$$

on $C$, which in turn finally produces the Mordell-Weil generator on $E$, with coordinates

$$
\begin{aligned}
& x=\frac{2263582143321421502100209233517777}{11981673410095561^{2}}, \\
& y=\frac{186398152584623305624837551485596770028144776655756}{11981673410095561^{3}} .
\end{aligned}
$$

Note that in the version given in the mailing list posting, the model was not minimal at 2 (in fact, it had level 2 at 2).

4-descent including minimisation and reduction was also used to find some of the elliptic curves of high rank and prescribed torsion listed in [Dujella n.d.], for example the curve with $E(\mathbb{Q}) \cong \mathbb{Z} / 12 \mathbb{Z} \times \mathbb{Z}^{4}$.

Minimised and reduced models of 2-, 3-, and 4-coverings provide the starting point for the computation of 6- and 12-coverings as described in [Fisher 2008c]. These then allow us to find even larger generators (of logarithmic canonical height $>600$ ). For example, this method was used to find the last missing generators for curves of prime conductor and rank at least 2 in the Stein-Watkins database [2002].

A table giving representatives of all elements of order 3 in the Shafarevich-Tate groups of all elliptic curves of conductor $<130000$ can be found at [Fisher n.d.]. (It is only known that the table is complete if one assumes the conjecture of Birch and Swinnerton-Dyer.) The final form of these ternary cubics was obtained by applying the methods described in this paper to the original models produced by the algorithms described in [Schaefer and Stoll 2004] and [Cremona et al. 2008; 2009; n.d.].

\section{References}

[An et al. 2001] S. Y. An, S. Y. Kim, D. C. Marshall, S. H. Marshall, W. G. McCallum, and A. R. Perlis, "Jacobians of genus one curves", J. Number Theory 90:2 (2001), 304-315. MR 2002g:14040 Zbl 1066.14035

[Artin et al. 2005] M. Artin, F. Rodriguez-Villegas, and J. Tate, "On the Jacobians of plane cubics", Adv. Math. 198:1 (2005), 366-382. MR 2006h:14043 Zbl 1092.14054

[Birch and Swinnerton-Dyer 1963] B. J. Birch and H. P. F. Swinnerton-Dyer, "Notes on elliptic curves. I”, J. Reine Angew. Math. 212 (1963), 7-25. MR 26 \#3669 Zbl 0118.27601

[Bosch et al. 1990] S. Bosch, W. Lütkebohmert, and M. Raynaud, Néron models, Ergebnisse der Math. (3) 21, Springer, Berlin, 1990. MR 91i:14034 
[Bosma et al. 1997] W. Bosma, J. Cannon, and C. Playoust, "The Magma algebra system, I: The user language", J. Symbolic Comput. 24:3-4 (1997), 235-265. MR 1484478

[Connell 1996] I. Connell, "Elliptic curve handbook", on-line notes, McGill University, 1996, available at http://www.math.mcgill.ca/connell/public/ECH1/.

[Cremona 1997] J. E. Cremona, Algorithms for modular elliptic curves, 2nd ed., Cambridge University Press, Cambridge, 1997. MR 99e:11068 Zbl 0872.14041

[Cremona 1999] J. E. Cremona, "Reduction of binary cubic and quartic forms", LMS J. Comput. Math. 2 (1999), 64-94. MR 2000f: 11040

[Cremona n.d.] J. E. Cremona, Elliptic curve data, available at http://www.warwick.ac.uk/staff/ J.E.Cremona/ftp/data/INDEX.html.

[Cremona et al. 2008] J. E. Cremona, T. A. Fisher, C. O’Neil, D. Simon, and M. Stoll, "Explicit n-descent on elliptic curves, I: Algebra", J. Reine Angew. Math. 615 (2008), 121-155. MR 2009g: 11067

[Cremona et al. 2009] J. E. Cremona, T. A. Fisher, C. O’Neil, D. Simon, and M. Stoll, "Explicit ndescent on elliptic curves, II: Geometry", J. Reine Angew. Math. 632 (2009), 63-84. MR 2544143

[Cremona et al. n.d.] J. E. Cremona, T. A. Fisher, C. O’Neil, D. Simon, and M. Stoll, "Explicit $n$-descent on elliptic curves, III: Algorithms", in preparation.

[Deligne 1975] P. Deligne, "Courbes elliptiques: formulaire d'après J. Tate”, pp. 53-73 in Modular functions of one variable, IV (Antwerp, 1972), edited by B. J. Birch and W. Kuyk, Lecture Notes in Math. 476, Springer, Berlin, 1975. MR 52 \#8135

[Djabri and Smart 1998] Z. Djabri and N. P. Smart, "A comparison of direct and indirect methods for computing Selmer groups of an elliptic curve", pp. 502-513 in Algorithmic number theory (Portland, OR, 1998), edited by J. Buhler, Lecture Notes in Comput. Sci. 1423, Springer, Berlin, 1998. MR 2001f:11086 Zbl 0915.11034

[Dolgachev 2003] I. Dolgachev, Lectures on invariant theory, London Mathematical Society Lecture Note Series 296, Cambridge University Press, Cambridge, 2003. MR 2004g:14051 Zbl 1023.13006

[Dujella n.d.] A. Dujella, "High rank elliptic curves with prescribed torsion", online table, available at http://web.math.hr/ duje/tors/tors.html.

[Fisher 2006a] T. Fisher, “The Hessian of a genus one curve”, preprint, 2006.

[Fisher 2006b] T. Fisher, "Testing equivalence of ternary cubics", pp. 333-345 in Algorithmic number theory, edited by F. Hess et al., Lecture Notes in Comput. Sci. 4076, Springer, Berlin, 2006. MR 2007j:11074 Zbl 1143.11325

[Fisher 2007] T. Fisher, "A new approach to minimising binary quartics and ternary cubics", Math. Res. Lett. 14:4 (2007), 597-613. MR 2008k:11058 Zbl 1142.11038

[Fisher 2008a] T. Fisher, “The invariants of a genus one curve", Proc. Lond. Math. Soc. (3) 97:3 (2008), 753-782. MR 2009j:11087 Zbl 05365466

[Fisher 2008b] T. Fisher, "Some improvements to 4-descent on an elliptic curve", pp. 125-138 in Algorithmic number theory, edited by A. van der Poorten and A. Stein, Lecture Notes in Comput. Sci. 5011, Springer, Berlin, 2008. MR 2009m:11078 Zbl 05279282

[Fisher 2008c] T. A. Fisher, "Finding rational points on elliptic curves using 6-descent and 12descent", J. Algebra 320:2 (2008), 853-884. MR 2009g:11068 Zbl 1149.14025

[Fisher n.d.] T. A. Fisher, "Elements of order 3 in the Tate-Shafarevich group", online table, available at http://www.dpmms.cam.ac.uk/ taf1000/g1data/order3.html.

[Gebel et al. 1998] J. Gebel, A. Pethő, and H. G. Zimmer, "On Mordell's equation”, Compositio Math. 110:3 (1998), 335-367. MR 98m:11049 Zbl 0899.11013 
[Hilbert 1993] D. Hilbert, Theory of algebraic invariants, Cambridge University Press, Cambridge, 1993. Translated by Reinhard C. Laubenbacher from handwritten course notes, taken by Sophus Marxsen. MR 97j:01049 Zbl 0801.13001

[Hodge and Pedoe 1952] W. V. D. Hodge and D. Pedoe, Methods of algebraic geometry, vol. II, Cambridge University Press, Cambridge, 1952. MR 95d:14002b Zbl 0048.14502

[Hulek 1986] K. Hulek, Projective geometry of elliptic curves, Astérisque 137, Société Mathématique de France, Paris, 1986. MR 88c:14046 Zbl 0602.14024

[Jacobson 1985] N. Jacobson, Basic algebra, I, 2nd ed., W. H. Freeman and Company, New York, 1985. MR 86d:00001 Zbl 0557.16001

[Julia 1917] G. Julia, "Étude sur les formes binaires non quadratiques à indeterminées réelles ou complexes”, Mem. Acad. Sci. l'Inst. France 55 (1917), 1-293.

[Kollár 1997] J. Kollár, "Polynomials with integral coefficients, equivalent to a given polynomial", Electron. Res. Announc. Amer. Math. Soc. 3 (1997), 17-27. MR 98g:11076 Zbl 0867.11047

[Kraus 1989] A. Kraus, "Quelques remarques à propos des invariants $c_{4}, c_{6}$ et $\Delta$ d'une courbe elliptique”, Acta Arith. 54:1 (1989), 75-80. MR 90j:11045 Zbl 0628.14024

[Laska 1982] M. Laska, "An algorithm for finding a minimal Weierstrass equation for an elliptic curve”, Math. Comp. 38:157 (1982), 257-260. MR 84e:14033 Zbl 0493.14016

[Lenstra et al. 1982] A. K. Lenstra, H. W. Lenstra, Jr., and L. Lovász, "Factoring polynomials with rational coefficients", Math. Ann. 261:4 (1982), 515-534. MR 84a:12002 Zbl 0488.12001

[Liu 1996] Q. Liu, "Modèles entiers des courbes hyperelliptiques sur un corps de valuation discrète", Trans. Amer. Math. Soc. 348:11 (1996), 4577-4610. MR 97h:11062 Zbl 0926.11043

[Merriman et al. 1996] J. R. Merriman, S. Siksek, and N. P. Smart, "Explicit 4-descents on an elliptic curve", Acta Arith. 77:4 (1996), 385-404. MR 97j:11027 Zbl 0873.11036

[Milne 2008] J. S. Milne, "Lectures on étale cohomology", v. 2.10, 2008, available at http:// www.jmilne.org/math/CourseNotes/LEC.pdf.

[Poonen 2001] B. Poonen, "An explicit algebraic family of genus-one curves violating the Hasse principle”, J. Théor. Nombres Bordeaux 13:1 (2001), 263-274. MR 2002e:14036 Zbl 1046.11038

[Raynaud 1970] M. Raynaud, Anneaux locaux henséliens, Lecture Notes in Math. 169, Springer, Berlin, 1970. MR 43 \#3252 Zbl 0203.05102

[Sadek 2009] M. Sadek, Models of genus one curves, Ph.D. thesis, University of Cambridge, 2009.

[Schaefer and Stoll 2004] E. F. Schaefer and M. Stoll, "How to do a $p$-descent on an elliptic curve", Trans. Amer. Math. Soc. 356:3 (2004), 1209-1231. MR 2004g:11045 Zbl 1119.11029

[Serre 1979] J.-P. Serre, Local fields, Graduate Texts in Mathematics 67, Springer, New York, 1979. MR 82e:12016 Zbl 0423.12016

[Siksek 1995] S. Siksek, Descent on curves of genus one, Ph.D. thesis, University of Exeter, 1995, available at http://www.warwick.ac.uk/staff/S.Siksek/papers/phdnew.pdf.

[Silverman 1986] J. H. Silverman, The arithmetic of elliptic curves, Graduate Texts in Mathematics 106, Springer, New York, 1986. MR 87g:11070 Zbl 0585.14026

[Silverman 1994] J. H. Silverman, Advanced topics in the arithmetic of elliptic curves, Graduate Texts in Mathematics 151, Springer, New York, 1994. MR 96b:11074 Zbl 0911.14015

[Skorobogatov 2001] A. Skorobogatov, Torsors and rational points, Cambridge Tracts in Mathematics 144, Cambridge University Press, Cambridge, 2001. MR 2002d:14032 Zbl 0972.14015

[Stein and Watkins 2002] W. A. Stein and M. Watkins, "A database of elliptic curves: first report", pp. 267-275 in Algorithmic number theory (Sydney, 2002), Lecture Notes in Comput. Sci. 2369, Springer, Berlin, 2002. MR 2005h:11113 Zbl 1058.11036 
[Stoll 2002] M. Stoll, Posting to NMBRTHRY mailing list, 2002, available at http://tinyurl.com/ 2 bgpxfd.

[Stoll and Cremona 2002] M. Stoll and J. E. Cremona, "Minimal models for 2-coverings of elliptic curves”, LMS J. Comput. Math. 5 (2002), 220-243. MR 2003j:11062 Zbl 1067.11031

[Stoll and Cremona 2003] M. Stoll and J. E. Cremona, "On the reduction theory of binary forms", J. Reine Angew. Math. 565 (2003), 79-99. MR 2005e:11091 Zbl 1153.11317

[Tate 1975] J. Tate, "Algorithm for determining the type of a singular fiber in an elliptic pencil", pp. 33-52 in Modular functions of one variable, IV (Antwerp, 1972), edited by B. J. Birch and W. Kuyk, Lecture Notes in Math. 476, Springer, Berlin, 1975. MR 52 \#13850

[Weil 1954] A. Weil, "Remarques sur un mémoire d'Hermite", Arch. Math. (Basel) 5 (1954), 197202. MR 15,896d Zbl 0056.03402

[Weil 1983] A. Weil, "Euler and the Jacobians of elliptic curves", pp. 353-359 in Arithmetic and geometry, I, edited by M. Artin and J. Tate, Progr. Math. 35, Birkhäuser, Boston, MA, 1983. MR 85d:14060 Zbl 0554.01014

[Womack 2003] T. Womack, Explicit descent on elliptic curves, Ph.D. thesis, University of Nottingham, 2003, available at http://www.warwick.ac.uk/staff/J.E.Cremona/theses/.

Communicated by Karl Rubin

Received 2010-01-19 Accepted 2010-07-18

J.E.Cremona@warwick.ac.uk Mathematics Institute, Zeeman Building, University of Warwick, Coventry, CV4 7AL, United Kingdom http://www.warwick.ac.uk/staff/J.E.Cremona

T.A.Fisher@dpmms.cam.ac.uk DPMMS, Centre for Mathematical Sciences, University of Cambridge, Wilberforce Road, Cambridge, CB3 OWB, United Kingdom http://www.dpmms.cam.ac.uk/ taf1000/

Michael.Stoll@uni-bayreuth.de Universität Bayreuth, Mathematisches Institut, 95440 Bayreuth, Germany http://www.mathe2.uni-bayreuth.de/stoll/ 


\section{Algebra \& Number Theory}

www.jant.org

\section{EDITORS}

\section{MANAGING EDITOR}

Bjorn Poonen

Massachusetts Institute of Technology

Cambridge, USA

\author{
EDITORIAL BOARD CHAIR \\ David Eisenbud \\ University of California \\ Berkeley, USA
}

\section{BOARD OF EDITORS}

\section{Georgia Benkart}

Dave Benson

Richard E. Borcherds

John H. Coates

J-L. Colliot-Thélène

Brian D. Conrad

Hélène Esnault

Hubert Flenner

Edward Frenkel

Andrew Granville

Joseph Gubeladze

Ehud Hrushovski

Craig Huneke

Mikhail Kapranov

Yujiro Kawamata

János Kollár

Hendrik W. Lenstra

Yuri Manin

Barry Mazur
University of Wisconsin, Madison, USA

University of Aberdeen, Scotland

University of California, Berkeley, USA

University of Cambridge, UK

CNRS, Université Paris-Sud, France

University of Michigan, USA

Universität Duisburg-Essen, Germany

Ruhr-Universität, Germany

University of California, Berkeley, USA

Université de Montréal, Canada

San Francisco State University, USA

Hebrew University, Israel

University of Kansas, USA

Yale University, USA

University of Tokyo, Japan

Princeton University, USA

Universiteit Leiden, The Netherlands

Northwestern University, USA

Harvard University, USA
Susan Montgomery

Shigefumi Mori

Andrei Okounkov

Raman Parimala

Victor Reiner

Karl Rubin

Peter Sarnak

Michael Singer

Ronald Solomon

Vasudevan Srinivas

J. Toby Stafford

Bernd Sturmfels

Richard Taylor

Ravi Vakil

Michel van den Bergh

Marie-France Vignéras

Kei-Ichi Watanabe

Andrei Zelevinsky

Efim Zelmanov
University of Southern California, USA

RIMS, Kyoto University, Japan

Princeton University, USA

Emory University, USA

University of Minnesota, USA

University of California, Irvine, USA

Princeton University, USA

North Carolina State University, USA

Ohio State University, USA

Tata Inst. of Fund. Research, India

University of Michigan, USA

University of California, Berkeley, USA

Harvard University, USA

Stanford University, USA

Hasselt University, Belgium

Université Paris VII, France

Nihon University, Japan

Northeastern University, USA

University of California, San Diego, USA

\section{PRODUCTION}

ant@mathscipub.org

Silvio Levy, Scientific Editor

Andrew Levy, Production Editor

See inside back cover or www.jant.org for submission instructions.

The subscription price for 2010 is US \$140/year for the electronic version, and \$200/year (+\$30 shipping outside the US) for print and electronic. Subscriptions, requests for back issues from the last three years and changes of subscribers address should be sent to Mathematical Sciences Publishers, Department of Mathematics, University of California, Berkeley, CA 94720-3840, USA.

Algebra \& Number Theory (ISSN 1937-0652) at Mathematical Sciences Publishers, Department of Mathematics, University of California, Berkeley, CA 94720-3840 is published continuously online. Periodical rate postage paid at Berkeley, CA 94704, and additional mailing offices.

ANT peer review and production are managed by EditFLOW ${ }^{\mathrm{TM}}$ from Mathematical Sciences Publishers.

\section{PUBLISHED BY}

mathematical sciences publishers

http://www.mathscipub.org

A NON-PROFIT CORPORATION

Typeset in LATEX

Copyright $\odot 2010$ by Mathematical Sciences Publishers 


\section{Algebra \& Number Theory}

Volume $4 \quad$ No. $6 \quad 2010$

Generalized moonshine I: Genus-zero functions

SCOTT CARNAHAN

Integral trace forms associated to cubic extensions

GUILLERMO MANTILLA-SOLER

RACHEL OLLIVIER

DANNY NEFTIN and ELAD PARAN

John E. Cremona, Tom A. Fisher and Michael Stoll 